\title{
WEIGHTED-BLOWUP CORRESPONDENCE OF ORBIFOLD GROMOV-WITTEN INVARIANTS AND APPLICATIONS
}

\author{
BOHUI CHEN, CHENG-YONG DU, AND JIANXUN HU
}

\begin{abstract}
Let $\mathrm{X}$ be a compact symplectic orbifold groupoid with $\mathrm{S}$ being a compact symplectic sub-orbifold groupoid, and $\underline{X}_{\mathfrak{a}}$ be the weight-a blowup of $X$ along $S$ with $Z$ being the exceptional divisor. We show that there is a weighted-blowup correspondence between some certain absolute orbifold Gromov-Witten invariants of $\mathrm{X}$ relative to $\mathrm{S}$ and some certain relative orbifold Gromov-Witten invariants of the pair $\left(\underline{X}_{\mathfrak{a}} \mid Z\right)$. As an application, we prove that the symplectic uniruledness of symplectic orbifold groupoids is a weighted-blowup invariant.
\end{abstract}

\section{CONTENTS}

1. Introduction 2

1.1. Weighted-blowup correspondence $\quad 3$

1.2. Symplectic uniruledness of orbifolds 4

Acknowledgements 4

2. Orbifolds as proper étale Lie groupoids 4

2.1. Proper étale Lie groupoids $\quad 5$

2.2. Space of morphisms and twisted sectors $\quad 7$

2.3. Groupoid actions and fiber bundles 9

2.4. Symplectic orbifold groupoids 10

3. Weighted blowups for symplectic orbifolds $\quad 10$

3.1. Weighted projective spaces 10

3.2. Weighted blowups of vector bundles 12

3.3. Weighted blowups of $X$ along $S \quad 14$

4. Orbifold Gromov-Witten theory 14

4.1. Absolute Gromov-Witten invariants 14

4.2. Relative Gromov-Witten invariants 15

4.3. The degeneration formula 16

4.4. Absolute Gromov-Witten invariants of symplectic pairs 17

5. Certain invariants of $\left(\bar{E}_{\mathfrak{a}} \mid P E_{\mathfrak{a}}\right) \quad 18$

5.1. Special case: $\mathrm{E}=\mathbb{Z}_{r}^{\mathfrak{b}} \ltimes \mathbb{C}^{n} \quad 18$

5.2. Computation of invariants 26

5.3. Special case: $E=\Gamma^{\mu} \ltimes \mathbb{C}^{n} \quad 31$

5.4. General cases: $E \rightarrow B \quad 32$

6. Weighted-blowup correspondence for orbifold Gromov-Witten invariants 35

6.1. Partial order on $\mathscr{R}_{\Sigma_{\star}}^{\bullet}(\mathrm{X} \mid \mathrm{Z})$

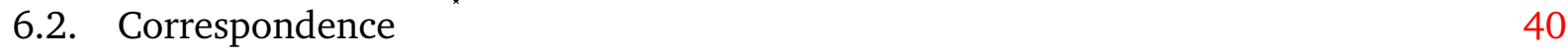

Date: July 15, 2019.

2010 Mathematics Subject Classification. 53D45; 14N35.

Key words and phrases. Weighted blowup; Orbifold correspondence; Symplectic uniruledness. 
7. Symplectic uniruledness

\begin{tabular}{ll} 
7.1. & Symplectic uniruledness of orbifold groupoids \\
\hline 7.2. & 47
\end{tabular}

7.2. Invariance of symplectic uniruledness under weighted blowups 48

7.3. ( $t$ )-symplectic uniruledness 48

$\begin{array}{ll}\text { References } & 49\end{array}$

\section{INTRODUCTION}

Symplectic birational geometry, proposed by Li-Ruan [34], concerns properties and structures of symplectic manifolds that are preserved by symplectic birational cobordisms. More explicitly, it studies properties and structures of symplectic manifolds that are captured by genus zero Gromov-Witten invariants. A symplectic birational cobordism consists of a finite sequence of symplectic reductions of Hamiltonian $S^{1}$-actions of (different) symplectic manifolds at (different) regular values, see Guillemin-Sternberg [19], Hu-Li-Ruan [24]. It was proved in [19] that a symplectic birational cobordism can be decomposed into a finite sequence of symplectic blowups/blow-downs and $\mathbb{Z}$-linear deformations of symplectic forms. Since Gromov-Witten invariants are deformation invariant, so the essential part is to discover the influence of symplectic blowups/blow-downs on the properties and structures captured by genus zero Gromov-Witten invariants. Therefore a beautiful and useful blowup formula for Gromov-Witten theory is a core to symplectic birational geometry. Gathmann [15] proved some blowup formulae of genus zero Gromov-Witten invariants for blowup of convex projective varieties along points. $\mathrm{Hu}[21,22]$ proved similar blowup formulae of genus zero and one Gromov-Witten invariants for blowup of symplectic manifolds along points, curves, and surfaces. When the ambient manifold has dimension less than 8, Hu's formulae hold for all genera.

A compact symplectic manifold $(X, \omega)$ is symplectic uniruled if it has a non-vanishing genus zero Gromov-Witten invariant

$$
\left\langle[p t], \alpha_{2}, \ldots, \alpha_{k}\right\rangle_{0, \beta}^{X} \neq 0
$$

with $0 \neq \beta \in H_{2}(X, \mathbb{Z})$ and $k \geq 1$. Kollár [28] and Ruan [40] proved that for a smooth projective uniruled variety $X$, there is a non-zero Gromov-Witten invariant as (1.1), i.e. $X$ is symplectic uniruled. It is well-known that projective uniruledness is a birational invariant. Therefore a natural question is that, if the symplectic uniruledness of symplectic manifolds is a symplectic birational cobordism invariant. By using those blow-up formulae proved by Gathmann and $\mathrm{Hu}$, one concludes that the blowup manifolds are symplectic uniruled provided that ambient manifolds are symplectic uniruled.

The big break-through for general symplectic blowup was made by $\mathrm{Hu}-\mathrm{Li}-\mathrm{Ruan}$ in [24]. Let $X$ be a compact symplectic manifold, $S \subseteq X$ be a compact symplectic submanifold, $\tilde{X}$ be the symplectic blowup of $X$ along $S$, and $E$ be the exceptional divisor. Hu-Li-Ruan [24] proved a general algorithm, known as a blowup correspondence of Gromov-Witten invariants, to compare certain relative Gromov-Witten invariants of $(\tilde{X} \mid E)$ and certain absolute GromovWitten invariants of $X$ relative to $S$. When $\operatorname{codim}_{\mathbb{C}} S=1$, i.e. $S$ is a symplectic divisor of $X$, such a blowup correspondence was obtained by Maulik-Pandharipande [36]. With this blowup correspondence Hu-Li-Ruan showed that symplectic uniruledness of symplectic manifolds is a symplectic birational cobordism invariant. 
Similarly, as a symplectic analog of rational connectedness in birational algebraic geometry one can also consider symplectic rational connectedness of symplectic manifolds. A compact symplectic manifold $(X, \omega)$ is symplectic rational connected if it has a non-vanishing genus zero Gromov-Witten invariant

$$
\left\langle[p t],[p t], \alpha_{3}, \ldots, \alpha_{k}\right\rangle_{0, \beta, k}^{X} \neq 0
$$

with $0 \neq \beta \in H_{2}(X, \mathbb{Z})$ and $k \geq 2$. Recently there are a lot of works on symplectic rational connectedness. For example, Li-Ruan [35], Hu-Ruan [25], Voisin [45], and Tian [43, 44] studied the symplectic rational connectedness of symplectic manifolds and projective varieties.

Orbifolds are natural generalization of manifolds. Roughly speaking orbifolds are manifolds with finite quotient singularities. Symplectic orbifolds appear naturally in symplectic reductions. It would be more convenient and challenging to involve orbifolds in symplectic birational geometry. This viewpoint was also addressed in [34]. We can adapt the definitions of symplectic uniruledness and symplectic rational connectedness of symplectic manifolds to symplectic orbifolds naturally. Then one may ask if symplectic uniruledness and symplectic rational connectedness of symplectic orbifolds are still birational invariants. There already have some works on symplectic uniruledness of orbifolds. $\mathrm{He}-\mathrm{Hu}$ [23] and $\mathrm{Du}$ [13] proved several formulae for orbifold Gromov-Witten invariants of weighted blowups along smooth points, which implies that when the ambient symplectic orbifold $X$ is symplectic uniruled then the weighted blowup $\underline{X}_{\mathfrak{a}}$ of $X$ along smooth points with weight $\mathfrak{a}$ is also symplectic uniruled. In this paper we study the change of Gromov-Witten invariants under weighted blowups of symplectic orbifolds along general symplectic sub-orbifolds. We show that symplectic uniruledness of symplectic orbifolds is an invariant under weighted blowups. This is a consequence of a weighted-blowup correspondence of orbifold Gromov-Witten invariants. We next describe the results.

1.1. Weighted-blowup correspondence. We first show a correspondence result for orbifold Gromov-Witten invariants under weighted blowups. Let $\underline{X}_{\mathfrak{a}}$ be the weight-a blowup of a symplectic orbifold groupoid $(X, \omega)$ along a symplectic sub-orbifold groupoid S. Denote the exceptional divisor by Z. let $\kappa: \underline{X}_{\mathfrak{a}} \rightarrow X$ be the natural projection associated to the blowup. By taking a self-dual basis $\sigma_{\star}$ of $H_{C R}^{*}(\mathrm{~S})$ we get a base $\Sigma_{\star}$ and a dual basis $\Sigma^{\star}$ of $H_{C R}^{*}(\mathrm{Z})$ (see $\S 5.4 .2$ ). We consider relative orbifold Gromov-Witten invariants of $\left(\underline{X}_{\mathfrak{a}} \mid Z\right)$ with relative insertions coming from $\Sigma_{\star}$ and absolute insertions coming from $\mathscr{K}:=\mid \kappa^{*}\left(H_{C R}^{*}(\mathrm{X})\right)$. We denote the set of admissible relative data (cf. $\S 4.2)$ by $\mathscr{R}_{\Sigma_{\star}, \mathscr{K}}\left(\underline{\mathrm{X}}_{\mathfrak{a}} \mid \mathrm{Z}\right)$. We assign a partial order to $\mathscr{R}_{\Sigma_{\star}, \mathscr{K}}\left(\underline{\mathrm{X}}_{\mathfrak{a}} \mid \mathrm{Z}\right)$ by using degeneration of moduli space associated to the degeneration of $\underline{X}_{\mathfrak{a}}$ along $Z$ with trivial weight (cf. $\S 6.1$ and Theorem 6.5). We use $\mathscr{R}_{\Sigma_{\star}, \mathscr{K}}\left(\underline{X}_{\mathfrak{a}} \mid Z\right)$ to generate a linear space $\mathbb{R}_{\Sigma_{\star}, \mathscr{K}}$. By using the Gromov-Witten invariants of relative data we get a vector $v_{\Sigma_{\star}, \mathscr{K}} \in \mathbb{R}_{\Sigma_{\star}, \mathscr{K}}$. We also consider absolute $\sigma_{\star}$-data of $(\mathrm{X}, \mathrm{S})$. In an admissible $\sigma_{\star}$-data $\mathbf{A}^{\bullet}\left(I ; I_{\mathrm{S}}\right)$ (cf. §4.1) we require that $I$ has no descendent insertions, i.e. $\psi$-classes, and $I_{S}$ are all supported on $S$, i.e. are of the form $\left(\sigma_{\star} \cup[\mathrm{IS}]\right) \psi^{c}$, where [IS] is the Thom class of the inertia space (cf. Definition 2.10) IS of $S$ in the inertia space IX of X. We denote the set of admissible absolute $\sigma_{\star}$-datum of $(X, S)$ by $\mathscr{A}_{\sigma_{\star}}(\mathrm{X}, \mathrm{S})$. For each admissible relative data $\mathbf{R}^{\bullet}(I \mid J) \in \mathscr{R}_{\Sigma_{\star}, \mathscr{K}}\left(\underline{X}_{\mathfrak{a}} \mid \mathrm{Z}\right)$ we associate it an admissible absolute data $\mathrm{A}^{\bullet}\left(I_{\kappa} ; I_{\mathrm{S}}\right) \in \mathscr{A}_{\sigma_{\star}}(\mathrm{X}, \mathrm{S})$. This gives us a map $\Psi: \mathscr{R}_{\Sigma_{\star}}\left(\underline{\mathrm{X}}_{\mathfrak{a}} \mid \mathrm{Z}\right) \rightarrow \mathscr{A}_{\sigma_{\star}}(\mathrm{X}, \mathrm{S})$, which is a bijection when $\operatorname{codim}_{\mathbb{C}} S>1$, and an injection when $\operatorname{codim}_{\mathbb{C}} S=1$, see Theorem 6.10. By using the absolute invariants of absolute data in the image of $\Psi$ we get another vector $v_{\sigma_{\star}} \in \mathbb{R}_{\Sigma_{\star}, \mathscr{K}}$. The map $\Psi$ gives us a linear map $L: \mathbb{R}_{\Sigma_{\star}, \mathscr{K}} \rightarrow \mathbb{R}_{\Sigma_{\star}, \mathscr{K}}$. We have

Theorem 1.1. (Theorem 6.12) The linear map $L: \mathbb{R}_{\Sigma_{\star}, \mathscr{K}} \rightarrow \mathbb{R}_{\Sigma_{\star}, \mathscr{K}}$ has the following properties: 
- $L\left(v_{\Sigma_{\star}, \mathscr{K}}\right)=v_{\sigma_{\star}}$,

- the matrix of $L$ with respect to the basis $\mathscr{R}_{\Sigma_{\star}, \mathscr{K}}\left(\underline{\mathrm{X}}_{\mathfrak{a}} \mid \mathrm{Z}\right)$ is lower triangle and has nonvanishing diagonals.

- $L$ is invertible.

We can restrict the correspondence $\Psi$ to genus zero relative/absolute datum which have a point class insertion as the first absolute insertion. Then we have a similar result (see Theorem 6.15 and Remark 6.16).

1.2. Symplectic uniruledness of orbifolds. In the manifold case, the motivation of symplectic uniruledness comes from the uniruledness in algebraic geometry which is about the existence of embedded rational curves passing through a given point, which leads to the equation (1.1). Now we consider the orbifold case. Let $X$ be a compact orbifold (cf. Remark 2.8 for compactness of orbifold). [pt] in (1.1) should be replaced by certain class in $H_{C R}^{*}(\mathrm{X})=H^{*}(\mathrm{IX})$. However, when the orbifold $X$ is ineffective, the situation is slightly complicated, which is explained below.

Let $\operatorname{ker} X=I X^{\text {top }}$ be the union of top dimensional twisted sectors of $X$ (cf. Remark 2.8 and Remark 2.11). The [pt] in (1.1) should be replaced by generators of $H^{\operatorname{dim} X}(k e r X)$. Note that when $X$ is effective, $\operatorname{ker} X=X(1) \cong X$. When $X$ is ineffective, ker $X$ has more than one component. This leads to the following definition. We say that a compact symplectic orbifold groupoid $X$ is symplectic uniruled if there is a nonzero orbifold Gromov-Witten invariant of $X$ of the form

$$
\left\langle[p t]_{\text {ker }}, \alpha_{2}, \ldots, \alpha_{k}\right\rangle_{0, A}^{\mathrm{X}} \neq 0
$$

with $k \geq 1$ and $0 \neq A \in H_{2}\left(|X| ; \mathbb{Z}\right.$ ) (see Definition 7.1 and Remark 7.2). Here $[p t]_{\text {ker }}$ depends on the location component of the point, and it runs through the generators of $H^{\text {dimX }}($ ker X).

One of the main application in this paper is the following

Theorem 1.2 (Theorem 7.4). Let $(X, \omega)$ be a compact symplectic orbifold groupoid with $\underline{X}_{a}$ being its weight-a blowup along a compact symplectic sub-orbifold $\mathrm{S}$. Then $\mathrm{X}$ is symplectic uniruled if and only if $\underline{X}_{\mathfrak{a}}$ is symplectic uniruled.

Furthermore, it is natural to ask whether there exists an embedded rational curve with orbifold structure passing through an orbifold point with given monodromy. That means, for symplectic orbifolds, we may consider a more general notion of symplectic uniruledness which involves point classes of general twisted sectors of $X$ not only ker $X$. We will discuss the invariance of this version of symplectic uniruledness in $\S 7.3$.

Acknowledgements. The authors thank the anonymous referee for careful reading and helpful suggestions, especially for the suggestion to consider the more general version of symplectic uniruledness discussed in §7.3. The first author is partially supported by NSFC (No. 11431001 \& No. 11890663). The second author is partially supported by NSFC (No. 11501393) and by Sichuan Science and Technology Program (No. 2019YJ0509). The third author is partially supported by NSFC (No. 11831017 \& No. 11890662 \& No. 11521101 \& No. 11771460).

\section{ORBIFOLDS AS PROPER ÉTALE LIE GROUPOIDS}

In this paper, we treat an orbifold as a Morita equivalence class of proper étale Lie groupoids, which are called orbifold groupoids. There are some nice references on orbifold groupoids, for example [2] and [39]. See also [5]. It is known that an effective orbifold is equivalent 
to a Morita equivalence class of effective proper étale Lie groupoids. But when effectiveness fails, orbifold groupoids should be used. Even for effective orbifolds, the structure of Lie groupoids provides a powerful way to control many tedious issues of orbifolds. In this section, we introduce the material of Lie groupoids needed in this paper.

\subsection{Proper étale Lie groupoids. We review some basic definitions.}

Definition 2.1 (Lie groupoids). A Lie groupoid $G$ consists of two smooth manifolds $G^{0}$ and $G^{1}$, together with five smooth maps $(s, t, m, u, i)$ satisfying the following properties.

(1) The source map and the target map $s, t: G^{1} \rightarrow G^{0}$ are both submersions.

(2) The composition map

$$
m: G^{[2]}:=\left\{\left(g_{1}, g_{2}\right) \in G^{1} \times G^{1}: t\left(g_{1}\right)=s\left(g_{2}\right)\right\} \rightarrow G^{1}
$$

written as $m\left(g_{1}, g_{2}\right)=g_{1} \circ g_{2}$ for composable elements $g_{1}$ and $g_{2}$, satisfies the obvious associative property.

(3) The unit map $u: G^{0} \rightarrow G^{1}$ is a two-sided unit for the composition.

(4) The inverse map $i: G^{1} \rightarrow G^{1}, i(g)=g^{-1}$, is a two-sided inverse for the composition.

For simplicity, we may denote $\mathrm{G}$ by $\mathrm{G}=\left(G^{1} \rightrightarrows G^{0}\right)$, where double arrows represent the source and target maps. $G^{0}$ is called the space of objects or units, and $G^{1}$ is called the space of arrows. An element $\alpha \in G^{1}$ is called an arrow from its source $s(\alpha)$ to its target $t(\alpha)$.

The Lie groupoid $\mathrm{G}$ is called proper if $(s, t): G^{1} \rightarrow G^{0} \times G^{0}$ is proper, and is called étale if $s$ and $t$ are local diffeomorphisms. A proper étale Lie groupoid is also called an orbifold groupoid.

Remark 2.2. $G^{1}$ defines an equivalence relation on $G^{0}$ : for $x, y \in G^{0}$ we say $x \sim y$ if and only if there exists an arrow from $x$ to $y$. Denote the quotient space $G^{0} / G^{1}$ by $|G|$, and denote the projection map by $\pi_{\mathrm{G}} .|\mathrm{G}|$ is called the coarse space of $\mathrm{G}$. Conversrly, $\mathrm{G}$ is called a Lie groupoid structure of the topological space $|\mathrm{G}|$. A Lie groupoid is connected, if its coarse space is connected.

Remark 2.3. A groupoid is a category in the following sense: $G^{0}$ is the set of objects; an arrow from object $x$ to $y$ is a morphism from $x$ to $y$; $m$ defines the composition of morphisms.

Definition 2.4. Let $\mathrm{G}$ and $\mathrm{H}$ be two Lie groupoids. By a strict morphism from $\mathrm{G}$ to $\mathrm{H}$ we mean a functor $\mathrm{f}: \mathrm{G} \rightarrow \mathrm{H}$ which consists of a pair of smooth maps $f^{i}: G^{i} \rightarrow H^{i}, i=0,1$ that are compatible with structure maps of groupoids. Every strict morphism $\mathrm{f}: \mathrm{G} \rightarrow \mathrm{H}$ induces a continuous map $|\mathrm{f}|:|\mathrm{G}| \rightarrow|\mathrm{H}|$ on coarse spaces.

A strict morphism $\mathrm{f}: \mathrm{G} \rightarrow \mathrm{H}$ is called an equivalence if

(1) the map to $\operatorname{proj}_{2}: G^{0} \times_{f^{0}, H^{0}, s} H^{1} \stackrel{\text { roj }_{2}}{\longrightarrow} H^{1} \stackrel{t}{\rightarrow} H^{0}$ is a surjective submersion;

(2) the square

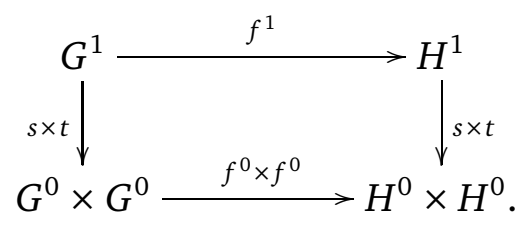

is a fiber product of smooth manifolds.

By a (weak) morphism from $\mathrm{G}$ to $\mathrm{H}$ we mean a triple $(\mathrm{g}, \mathrm{M}, \mathrm{f})$ with the diagram

$$
\mathrm{G} \stackrel{\mathrm{g}}{\longleftarrow} \mathrm{M} \stackrel{\mathrm{f}}{\rightarrow} \mathrm{H},
$$

where $\mathrm{g}: \mathrm{M} \rightarrow \mathrm{G}$ is an equivalence and $\mathrm{f}$ is a strict morphism. 
We give some typical examples of Lie groupoids that will be used in this paper.

Example 2.5 (Quotient groupoids). Let $M$ be a smooth manifold and $G$ be a Lie group. Suppose that $G$ acts on $M$ smoothly with the action map $\phi: M \times G \rightarrow M$. The quotient space $M / G$ admits a Lie groupoid structure

$$
G \ltimes M:=(M \times G \rightrightarrows M),
$$

where $s(m, g)=m$ and $t=\phi$. This is called the quotient groupoid for $M / G$.

The following are special cases.

(1) By identifying a manifold $M$ as a quotient groupoid $M /\{e\}$, hence $\{e\} \ltimes M \cong(M \rightrightarrows M)$ gives a canonical groupoid structure on $M$;

(2) for any group $G$ acting trivially on a point $\{p t\}$, we denote the quotient groupoid by $B G$;

(3) for any positive integer $r$, we denote $B \mathbb{Z}_{r}$ by $B_{r}$; in this sense, $B_{1}=\{p t\}$. In this paper, we identify

$$
\mathbb{Z}_{r}=\left\langle\zeta_{r}\right\rangle
$$

where $\zeta_{r}=e^{2 \pi i \frac{1}{r}}$.

Example 2.6. Let $\mathrm{G}=\left(G^{1} \rightrightarrows G^{0}\right)$ be a Lie groupoid. For any open subset $U^{0} \subseteq G^{0}$, define

$$
U^{1}=s^{-1}\left(U^{0}\right) \cap t^{-1}\left(U^{0}\right) .
$$

Then we call ( $\left.U^{1} \rightrightarrows U^{0}\right)$ is a groupoid induced on $U^{0}$; we denote it by $\mathrm{G}\left(U^{0}\right)$ or $\left.\mathrm{G}\right|_{U^{0}}$.

For any open subset $\mathrm{U} \subseteq|\mathrm{G}|$, let $U^{0}=\pi_{\mathrm{G}}^{-1}(\mathrm{U})$. Then we have an induced groupoid $\mathrm{G}\left(U^{0}\right)=$ $\left(U^{1} \rightrightarrows U^{0}\right)$ for $\mathbf{U}$.

In fact, for any open subset $U^{0} \subseteq G^{0}, \mathrm{G}\left(U^{0}\right)$ is equivalent to $\mathrm{G}\left(\pi_{\mathrm{G}}^{-1}\left(\pi_{\mathrm{G}}\left(U^{0}\right)\right)\right.$, that is the natural inclusion $\mathrm{G}\left(U^{0}\right) \hookrightarrow \mathrm{G}\left(\pi_{\mathrm{G}}^{-1}\left(\pi_{\mathrm{G}}\left(U^{0}\right)\right)\right.$ is an equivalence of Lie groupoids.

When $\mathrm{U}$ is a connected component of $|\mathrm{G}|$ we call $\mathrm{G}\left(\pi_{\mathrm{G}}^{-1}(\mathrm{U})\right)$ a connected component of $\mathrm{G}$.

Remark 2.7. Let $\mathrm{G}$ be a proper étale Lie groupoid. We have following properties.

(1) For every $x \in G^{0}$,

$$
G_{x}:=(s, t)^{-1}(x, x)=s^{-1}(x) \cap t^{-1}(x)
$$

is a finite group. We call $G_{x}$ to be the isotropy group at $x$.

(2) When $\mathrm{G}$ is effective (cf. [2, Definition 1.38]), there exists a small neighborhood $U^{0}$ of $x$ such that the induced groupoid structure

$$
\mathrm{G}\left(U^{0}\right)=G_{x} \ltimes U^{0},
$$

which is the groupoid structure for $\mathbf{U}=\pi_{\mathrm{G}}\left(U^{0}\right)$. It is also called an orbifold chart over $\mathbf{U}$ or a local orbifold chart around $x$.

This leads to the following definition which is given in [2]: a proper étale Lie groupoid $\mathrm{G}$ is an orbifold structure of the topological space $X=|\mathrm{G}|$. A space $X$ is called an orbifold if it admits a proper etále Lie groupoid as its orbifold structure.

Remark 2.8. Let $G=\left(G^{1} \rightrightarrows G^{0}\right)$ be a connected proper étale Lie groupoid. Let ker $\mathrm{G}$ be the space of constant arrows (or ineffective arrows) (cf. [2, Definition 2.27]). It is showed in [2, Lemma 2. 32] that ker $G$ consists of a union of connected components in $G^{1}$. G acts on ker $G$ (cf. Definition 2.14) naturally by conjugations. We denote the resulting action groupoid $\mathrm{G} \ltimes$ ker $\mathrm{G}$ by ker $\mathrm{G}$. It is also proper étale. However, it may not be connected. When $\mathrm{G}$ is effective, $\operatorname{ker} \mathrm{G} \cong \mathrm{G}$ is connected. Otherwise ker $\mathrm{G}$ has several connected component and we call $\mathrm{G}$ an ineffective orbifold groupoid. 
In this paper we say that a proper étale Lie groupoid $\mathrm{G}$ is compact if the coarse space |ker $\mathrm{G} \mid$ of $\operatorname{ker} \mathrm{G}$ is compact. In this circumstance, local orbifold charts also exist for ineffective orbifold groupoids.

In the following we only consider connected compact proper étale Lie groupoids.

2.2. Space of morphisms and twisted sectors. Let $G=\left(G^{1} \rightrightarrows G^{0}\right)$ and $H=\left(H^{1} \rightrightarrows H^{0}\right)$ be two Lie groupoids. Let $\operatorname{SMor}(H, G)$ be the space of strict morphisms from $H$ to $G$. For any two morphisms $f, g \in \operatorname{SMor}(H, G)$, we say that they are equivalent, denote as $f \sim g$, if there is a natural transformation $\phi: f \Rightarrow g$, i.e, a smooth map $\phi: H^{0} \rightarrow G^{1}$, such that

$$
s \circ \phi(x)=f^{0}(x), \quad f^{1}(\alpha) \circ \phi(t(\alpha))=\phi(s(\alpha)) \circ g^{1}(\alpha)
$$

for any $x \in H^{0}$ and $\alpha \in H^{1}$. Let $\operatorname{SMor}(H, G)$ be the quotient space $\operatorname{SMor}(H, G) / \sim$.

$\operatorname{SMor}(H, G)$ admits a natural groupoid structure, denoted by

$$
\operatorname{SMor}(H, G)=\left(\operatorname{SMor}^{1}(H, G) \rightrightarrows \operatorname{SMor}^{0}(H, G)\right) \text {, }
$$

where $\operatorname{SMor}^{0}(H, G)=\operatorname{SMor}(H, G)$ and $\operatorname{SMor}^{1}(H, G)$ consists of all natural transformations between any two morphisms. A natural transformation from $f$ to $g$ is called an arrow from $f$ to g.

Remark 2.9. We make some remarks.

(1) $\operatorname{SMor}^{0}(H, G)$ can be only a topological space even with proper Sobolev norms, hence $\operatorname{SMor}(\mathrm{H}, \mathrm{G})$ is only a topological groupoid when proper topology is endowed.

(2) If $\mathrm{G}$ and $\mathrm{H}$ are proper and étale, so is $\operatorname{SMor}(\mathrm{H}, \mathrm{G})$;

(3) In order to consider all possible morphisms from $\mathrm{H}$ to $\mathrm{G}$, one must consider weak morphisms. This leads to a groupoid $\operatorname{Mor}(\mathrm{H}, \mathrm{G})$ (cf. [4]). If $\mathrm{H}$ and $\mathrm{G}$ are two proper étale Lie groupoids, Mor $(\mathrm{H}, \mathrm{G})$ is equivalent to a proper etále Banach Lie groupoid when proper Sobolev norms are adapted.

Let $\mathrm{G}$ be an orbifold groupoid. Take $\mathrm{H}=\mathrm{B}_{r}$ (cf. Example 2.5). Let

$$
\operatorname{IMor}^{0}\left(B_{r}, G\right)=\left\{f=\left(f^{0}, f^{1}\right) \mid f^{1} \text { is injective }\right\} \subseteq \operatorname{SMor}^{0}\left(B_{r}, G\right) \text {. }
$$

Hence every $\mathrm{f}=\left(f^{0}, f^{1}\right) \in \operatorname{IMor}^{0}\left(B_{r}, G\right)$ corresponds to a monomorphism $f^{1}: \mathbb{Z}_{r} \hookrightarrow G_{f^{0}(p t)}$. Then $\operatorname{SMor}\left(\mathrm{B}_{r}, \mathrm{G}\right)$ induces a Lie groupoid

$$
\operatorname{IMor}_{r}(\mathrm{G}):=\left(\operatorname{IMor}^{1}\left(\mathrm{~B}_{r}, \mathrm{G}\right) \rightrightarrows \operatorname{IMor}^{0}\left(\mathrm{~B}_{r}, \mathrm{G}\right)\right)=\left.\operatorname{SMor}\left(\mathrm{B}_{r}, \mathrm{G}\right)\right|_{\operatorname{IMor}^{0}\left(\mathrm{~B}_{r}, \mathrm{G}\right)},
$$

which is proper and étale. So

$$
\operatorname{IMor}^{1}\left(B_{r}, G\right)=\left\{\sigma: f_{1} \Rightarrow f_{2} \mid f_{1}, f_{2} \in \operatorname{IMor}^{0}\left(B_{r}, G\right)\right\},
$$

and every natural transformation $\sigma: f_{1}=\left(f_{1}^{0}, f_{1}^{1}\right) \Rightarrow f_{2}=\left(f_{2}^{0}, f_{2}^{1}\right)$ corresponds to an arrow $\sigma(p t): f_{1}^{0}(p t) \rightarrow f_{2}^{0}(p t)$ that satisfies

$$
\sigma(p t)^{-1} \circ f_{1}^{1}\left(\zeta_{r}\right) \circ \sigma(p t)=f_{2}^{1}\left(\zeta_{r}\right) .
$$

Definition 2.10. The proper étale Lie groupoid

$$
\mathrm{IG}=\bigsqcup_{r \in \mathbb{Z}^{+}} \mathrm{IMor}_{r}(\mathrm{G})
$$

is called the inertia space of $\mathrm{G}$. 
There is a natural morphism $e: I G \rightarrow G$ called the evaluation morphism, which on objects maps each $\mathrm{f}=\left(f^{0}, f^{1}\right) \in \operatorname{IMor}^{0}\left(\mathrm{~B}_{r}, \mathrm{G}\right)$ to $f^{0}(p t)$ and on arrows maps each $\sigma \in \operatorname{IMor}^{1}\left(\mathrm{~B}_{r}, \mathrm{G}\right)$ to $\sigma(p t)$.

According to the decomposition of the coarse space $|I G|$ into connected components, IG decompose into connected components. We call each connected component a twisted sector of $\mathrm{G}$. We say that a twisted sector is of top dimension, if the restriction of $e: I G \rightarrow G$ is a local diffeomorphism on object spaces. Denote the union of top dimensional twisted sectors by $I G^{\text {top }}$.

Remark 2.11. We have $I \mathrm{G}^{\text {top }}=\operatorname{ker} \mathrm{G}$. Therefore $\operatorname{ker} \mathrm{G}$ is a (possibly union of) top dimensional twisted sector of $\mathrm{G}$.

Example 2.12 (Inertia space of quotient groupoids). Suppose that $\mathrm{G}=M \rtimes \Gamma$ is a quotient groupoid. Then

$$
\operatorname{IMor}_{r}(\mathrm{G})=\underset{(\gamma), \operatorname{ord}(\gamma)=r}{C_{\Gamma}} C_{(\gamma) \ltimes M^{\gamma},}
$$

where $(\gamma)$ is a conjugacy class of $\gamma, M^{\gamma}$ is the space fixed by $\gamma, C_{\Gamma}(\gamma)$ is the centralizer of $\gamma$.

Remark 2.13. Now we give a local description of twisted sectors of a compact orbifold groupoid G. Let $x \in G^{0}$ and $G_{x} \times U^{0}$ be a local orbifold chart. For any $g \in G_{x}$, there is a twisted sector for the conjugate class $(g)$ which is locally given by $C_{G_{x}}(g) \ltimes\left(U^{0}\right)^{g}$. In [11], $(g)$ is treated as the index for the twisted sector. For instance we have $\mathrm{G}(1)$, where 1 is the unit element in isotropy groups. Moreover, e $: \mathrm{IG} \rightarrow \mathrm{G}$ restricts to an isomorphism $\mathrm{G}(1) \cong \mathrm{G}$.

Let $\mathscr{T}^{\mathrm{G}}$ be the set of indices of twisted sectors. A twisted sector is denoted by $\mathrm{G}(\delta)$ for $\delta \in \mathscr{T}^{\mathrm{G}}$. The restriction of $e: I G \rightarrow G$ on a twisted sector $\mathrm{G}(\delta)$ is denoted by $e_{\delta}$. For convenience, when we use local models, we may abuse the notations to treat $(g)$ as an element of $\mathscr{T}^{\mathrm{G}}$. We set $\mathscr{T}_{\mathrm{ker}}^{\mathrm{G}}$ to be the index set of top dimensional twisted sectors. Then

$$
\operatorname{ker} G=I G^{\text {top }}=\bigsqcup_{\delta \in \mathscr{T}_{\text {ker }}^{G}} G(\delta) .
$$

When $\mathrm{G}$ is effective, $\operatorname{ker} \mathrm{G}=\mathrm{G}(1) \cong \mathrm{G}$ via e.

The Chen-Ruan cohomology of an orbifold groupoid $\mathrm{G}$ is defined to be

$$
H_{C R}^{*}(\mathrm{G})=H^{*}(\mathrm{IG})
$$

with proper degree shiftings. In [11], Chen and Ruan introduced a natural Poincaré pairing for $H_{C R}^{*}$. We recall the definition. For a morphism $f: B_{r} \rightarrow G$ given by

$$
f^{0}: p t \mapsto x, f^{1}: \zeta_{r} \mapsto \gamma \in G_{x}
$$

define a "dual" morphism $\overline{\mathrm{f}}: \mathrm{B}_{r} \rightarrow \mathrm{G}$ given by

$$
\bar{f}^{0}: p t \mapsto x, \quad \bar{f}^{1}: \zeta_{r} \mapsto \gamma^{-1} \in G_{x} .
$$

This induces a morphism ${ }^{-}: I G \rightarrow I G$ and also

$$
\bar{r}: \mathscr{T}^{\mathrm{G}} \rightarrow \mathscr{T}^{\mathrm{G}} .
$$

The Poincaré pairing on $H_{C R}^{*}(\mathrm{G})$ is defined to be

$$
\langle\cdot, \cdot\rangle: H^{*}(\mathrm{G}(\delta)) \times H^{*}(\mathrm{G}(\bar{\delta})) \rightarrow \mathbb{R}, \quad\langle\alpha, \beta\rangle=\int_{\mathrm{G}(\delta)} \alpha \wedge^{* *}(\beta)
$$

for all $\delta \in \mathscr{T}^{\mathrm{G}}$. 


\subsection{Groupoid actions and fiber bundles.}

Definition 2.14 (Groupoid actions). A right action of $\mathrm{G}$ on a space $X^{0}$ consists of two smooth maps $\pi^{0}: X^{0} \rightarrow G^{0}$ and

$$
\begin{aligned}
\phi: X^{1}:=X^{0} \times_{\pi^{0}, G^{0}, s} G^{1}=\left\{(x, g) \mid \pi^{0}(x)=s(g)\right\} & \rightarrow X^{0}, \\
(x, g) & \mapsto x g
\end{aligned}
$$

such that

$$
(x g) h=x(g h), \quad x u\left(\pi^{0}(x)\right)=x, \quad \pi^{0}(x g)=t(g) .
$$

We call $\pi^{0}$ the base map, and $\phi$ the action map.

We associate a groupoid $\left(X^{1} \rightrightarrows X^{0}\right)$ to the action by setting the groupoid structure maps to be

$$
\begin{gathered}
s(x, g)=x, t(x, g)=x g, \\
m((x, g),(x g, h))=(x, g h), u(x)=\left(x, u\left(\pi^{0}(x)\right)\right), i(x, g)=\left(x g, g^{-1}\right) .
\end{gathered}
$$

We denote this groupoid by $\mathrm{G} \ltimes X^{0}$, and call it the action groupoid associated to the G-action on $X^{0}$.

Let $\pi^{1}: X^{1} \rightarrow G^{1}$ be the projection. Then $\pi=\left(\pi^{0}, \pi^{1}\right): \mathrm{G} \ltimes X^{0} \rightarrow \mathrm{G}$ is a strict morphism. We call it the projection of the groupoid action.

Definition 2.15 (Fiber bundles). By a fiber bundle over $\mathrm{G}$ with fiber $F$ we mean a strict morphism $\pi: \mathrm{E} \rightarrow \mathrm{G}$, such that

(1) $\pi^{0}: E^{0} \rightarrow G^{0}$ is a fibration with fiber $F$;

(2) $\mathrm{E}=\mathrm{G} \ltimes E^{0}$ for some $\mathrm{G}$-action on $E^{0}$, and $\pi$ is the projection of the groupoid action.

We call $\mathrm{E}$ to be a vector bundle over $\mathrm{G}$ if $\pi^{0}: E^{0} \rightarrow G^{0}$ is a vector bundle and the action map $\phi(x, g)$ is linear in $x$.

As the bundle theory over manifolds, we introduce the concepts of principal bundle and associated bundle.

Definition 2.16 (Principal bundles). Let $K$ be a Lie group and $\pi: \mathrm{P} \rightarrow \mathrm{B}$ be a K-fiber bundle. We say that $\mathrm{P}$ is a $\mathrm{K}$-principal bundle if $\pi^{0}: P^{0} \rightarrow B^{0}$ is a (left) K-principal bundle and the $K$-action on $P^{0}$ commutes with the B-action.

For a principal $K$-bundle $\pi: P \rightarrow B, \pi^{i}: P^{i} \rightarrow B^{i}, i=0,1$ are left $K$-principal bundles. Then

$$
K \backslash \mathrm{P}:=\left(K \backslash P^{1} \rightrightarrows K \backslash P^{0}\right) \cong \mathrm{B} .
$$

For any right $K$-space $F$, we may define an associated bundle

$$
F \times{ }_{K} \mathrm{P}=\left(F \times{ }_{K} P^{1} \rightrightarrows F \times{ }_{K} P^{0}\right)
$$

This can be generalized by allowing that $F$ is a groupoid. Suppose that $F=\left(F^{1} \rightrightarrows F^{0}\right)$ admits a group $K$-action, then

$$
\mathrm{F} \times_{K} \mathrm{P}:=\left(F^{1} \times_{K} P^{1} \rightrightarrows F^{0} \times_{K} P^{0}\right)
$$

Remark 2.17. The right and left actions defined above can be switched to be left and right ones. 
2.4. Symplectic orbifold groupoids. Let $X=\left(X^{1} \rightrightarrows X^{0}\right)$ be an orbifold groupoid. By a symplectic form $\omega$ on $X$ we mean a pair of symplectic forms $\left(\omega^{0}, \omega^{1}\right)$ with $\omega^{i} \in \Omega^{2}\left(X^{i}\right), i=0,1$, such that $s^{*} \omega^{0}=t^{*} \omega^{0}=\omega^{1}$. We call $(\mathrm{X}, \omega)$ a symplectic orbifold groupoid if $\omega$ is a symplectic form on $X$. A strict morphism $f=\left(f^{1}, f^{0}\right):(X, \omega) \rightarrow(Y, \eta)$ is called a symplectomorphism if both $f^{0}$ and $f^{1}$ are symplectomorphisms, and then we call that $(\mathrm{X}, \omega)$ is symplectic isomorphic to $(Y, \eta)$.

We say that an orbifold groupoid $S=\left(S^{1} \rightrightarrows S^{0}\right)$ is a sub-orbifold groupoid of $X$ if $S^{0}$ is a smooth submanifold of $X^{0}$ and $S^{1}=s^{-1}\left(S^{0}\right) \cap t^{-1}\left(S^{0}\right)$, hence $S^{1}$ is also a smooth submanifold of $X^{1}$. We call $S$ to be a symplectic sub-orbifold groupoid of $(X, \omega)$ if $S^{i}, i=0,1$ are symplectic submanifolds of $\left(X^{i}, \omega^{i}\right)$. In this case we call $(X, S)$ a symplectic orbifold groupoid pair. We also have the following symplectic neighborhood theorem.

Theorem 2.18 ([14]). Let $(X, S)$ be a symplectic orbifold groupoid pair, and suppose that $S$ is compact. Then there is a symplectic sub-orbifold groupoid $\mathrm{S}_{*}=\left(S_{*}^{1} \rightrightarrows S_{*}^{0}\right) \subseteq \mathrm{S}$ of X such that

- the natural inclusion $S_{*} \hookrightarrow S$ is an equivalence in the sense of Definition 2.4;

- there is a tubular neighborhood $\mathrm{U}=\left(U^{1} \rightrightarrows U^{0}\right)$ of $\mathrm{S}_{*}$ in $\mathrm{X}$, i.e. $\mathrm{U}$ is an (open) suborbifold groupoid of $X$ and $U^{i}$ are tubular neighborhood of $S_{*}^{i}$ in $X^{i}$;

- $\left(\mathrm{U},\left.\omega\right|_{\mathrm{U}}\right)$ is symplectic isomorphic to a disc bundle of the normal bundle $\mathrm{N}$ (with the induced symplectic form) of $\mathrm{S}_{*}$ in $\mathrm{X}$.

Then we can take compatible almost complex structures on both $\mathrm{N}$ and $\mathrm{U}$ such that the symplectomorphism $\mathrm{U} \rightarrow \mathrm{N}$ also preserves the almost complex structures.

We see that $U^{i}$ are symplectic tubular neighborhood of $S_{*}^{i}$ in $\left(X^{i}, \omega_{i}\right)$, which are symplectomorphic to disc bundle of the normal bundle $N^{i}$ of $S_{*}^{i}$ in $X^{i}$ for $i=1,2$.

\section{WEIGHTED BLOWUPS FOR SYMPLECTIC ORBIFOLDS}

Let $(X, S)$ be a symplectic orbifold groupoid pair. We will discuss the weighted blowups of $\mathrm{X}$ along S. By Theorem 2.18, it is sufficient to discuss the weighted blowups of vector bundles along their 0-sections.

3.1. Weighted projective spaces. Suppose $S^{1}$ acts on $\mathbb{C}^{n}$ with weight $\mathfrak{a}=\left(\alpha_{1}, \ldots, \alpha_{n}\right), \alpha_{i} \in$ $\mathbb{Z}_{>0}$. Namely,

$$
t \cdot\left(z_{1}, \ldots, z_{n}\right)=\left(t^{\alpha_{1}} z_{1}, \ldots, t^{\alpha_{n}} z_{n}\right) .
$$

For simplicity, we denote the right hand side by $t^{\mathfrak{a}} \cdot z$ and denote this action by $S_{\mathfrak{a}}^{1}$. The quotient groupoid $S_{\mathfrak{a}}^{1} \ltimes S^{2 n-1}$ is called a weight-a projective space, and is denoted by $\mathrm{P}_{\mathfrak{a}}$. We say that $P_{\mathfrak{a}}$ is the weight-a projectivization of $\mathbb{C}^{n}$ with respect to the $S_{\mathfrak{a}}^{1}$ action.

The following concepts are related:

(1) the weight-a projectification of $\mathbb{C}^{n}$ with respect to the $S_{\mathfrak{a}}^{1}$ action is defined to be

$$
\left[\overline{\mathbb{C}^{n}}\right]_{\mathfrak{a}}:=\mathrm{P}_{(\mathfrak{a}, 1)}=S_{(\mathfrak{a}, 1)}^{1} \ltimes S^{2 n+1},
$$

where $S_{(\mathfrak{a}, 1)}^{1}$ acts as $t \cdot(z, w)=\left(t^{\mathfrak{a}} z, t w\right)$. Hence $P_{(\mathfrak{a}, 1)}$ is obtained by adding $P_{\mathfrak{a}}$ to $\mathbb{C}^{n}$ at infinity.

(2) the weight-a blowup of $\mathbb{C}^{n}$ at origin is defined to be

$$
\left[\underline{\mathbb{C}^{n}}\right]_{\mathfrak{a}}:=S^{2 n-1} \times_{S^{1}(\mathfrak{a},-1)} \mathbb{C},
$$


where $S^{1}$ acts on $S^{2 n-1}$ and $\mathbb{C}$ with weights $\mathfrak{a}$ and -1 respectively. There is a natural map

$$
\kappa:\left[\underline{\mathbb{C}^{n}}\right]_{\mathfrak{a}} \rightarrow \mathbb{C}^{n} ; \quad[z, w] \mapsto w^{\mathfrak{a}} \cdot z,
$$

where $\kappa^{-1}(0)=P_{\mathfrak{a}}$ is called the exceptional divisor.

Let $\Gamma$ be a finite group. Suppose that it acts on $\mathbb{C}^{n}$ via the representation

$$
\mu: \Gamma \rightarrow G L(n, \mathbb{C})
$$

and it commutes with the $S_{\mathfrak{a}}^{1}$ action. Then we have following constructions for the orbifold $\Gamma^{\mu} \ltimes \mathbb{C}^{n}$ (here, we write $\Gamma^{\mu}$ for $\Gamma$ ):

$$
\mathrm{P}_{\mathfrak{a}}^{\mu}=\mathrm{P}_{\mathfrak{a}} / \Gamma^{\mu}, \quad\left[\overline{\mathbb{C}^{n}}\right]_{\mathfrak{a}}^{\mu}=\left[\overline{\mathbb{C}^{n}}\right]_{\mathfrak{a}} / \Gamma^{\mu}, \quad\left[\underline{\mathbb{C}^{n}}\right]_{\mathfrak{a}}^{\mu}=\left[\underline{\mathbb{C}^{n}}\right]_{\mathfrak{a}} / \Gamma^{\mu}
$$

In particular, when $\Gamma=\mathbb{Z}_{r}$ and the action weight is

$$
\mathfrak{b}=\left(\beta_{1}, \ldots, \beta_{n}\right), \quad \text { where } 1 \leq \beta_{1}, \ldots, \beta_{n} \leq r,
$$

we denote these spaces as $P_{\mathfrak{a}}^{\mathfrak{b}},\left[\overline{\mathbb{C}^{n}}\right]_{\mathfrak{a}}^{\mathfrak{b}}$ and $\left[\underline{\mathbb{C}^{n}}\right]_{\mathfrak{a}}^{\mathfrak{b}}$ respectively.

Remark 3.1. Note that the range of $\beta$ is taken to be $[1, r]$ rather than $[0, r-1]$.

We give an explicit description of the inertia space of $\mathrm{P}_{\mathfrak{a}}^{\mu}$. Since

$$
\mathrm{P}_{\mathfrak{a}}^{\mu}=\left(\Gamma^{\mu} \times S_{\mathfrak{a}}^{1}\right) \ltimes S^{2 n-1},
$$

its twisted sectors are parameterized by conjugate classes $(\delta), \delta \in \Gamma \times S^{1}$. The inertia space is

$$
\mathrm{IP}_{\mathfrak{a}}^{\mu}=\bigsqcup_{(\delta), \delta \in \Gamma \times S^{1}}\left(S^{2 n-1}\right)^{\delta} \ltimes C_{\Gamma \times S^{1}}(\delta) .
$$

Be precise, suppose that $\delta=(\eta, t)$, then

$$
C_{\Gamma \times S^{1}}(\delta)=C_{\Gamma}(\eta) \times S^{1}
$$

Denote the fixed point set of $\delta$ by

$$
\mathbb{C}^{(\delta)}=\left(\mathbb{C}^{n}\right)^{\delta}, \quad S^{(\delta)}=\left(S^{2 n-1}\right)^{\delta}
$$

Then

$$
\mathrm{P}_{\mathfrak{a}}^{\mu}(\delta)=\frac{S^{(\delta)}}{C_{\Gamma}(\eta) \times S_{\mathfrak{a}(\delta)}^{1}}
$$

where $\mathfrak{a}(\delta)$ is the action weight on $\mathbb{C}^{(\delta)}$.

Now suppose that $\Gamma=\mathbb{Z}_{r}=\left\langle\zeta_{r}\right\rangle$ with $\zeta_{r}=e^{2 \pi i \frac{1}{r}}$ and the action weight is $\mathfrak{b}=\left(\beta_{1}, \ldots, \beta_{r}\right)$, i.e,

$$
\zeta_{r} \cdot\left(z_{1}, \ldots, z_{n}\right)=\left(\zeta_{r}^{\beta_{1}} z_{1}, \ldots, \zeta_{r}^{\beta_{n}} z_{n}\right)
$$

We give an explicit description for its twisted sectors. Let $\mathrm{p}_{i}=\left[\ldots, 0, z_{i}=1,0, \ldots\right]$. Denote the isotropy group of $\mathrm{p}_{i}$ by $G_{i}$. Then

$$
G_{i}=\left\{\left(e^{-2 \pi i \frac{b}{r}}, e^{2 \pi i \frac{b \beta_{i}+a r}{\alpha_{i} r}}\right) \mid 0 \leq b \leq r-1,0 \leq a \leq \alpha_{i}-1\right\} .
$$

We denote the group elements by $g_{i, a, b}$. We list some easy lemma without proofs.

Lemma 3.2. The index set of twisted sectors of $P_{\mathfrak{a}}^{\mathfrak{b}}$

$$
\mathscr{T} \subseteq \mathbb{Z}_{r} \times S^{1}
$$

is bijective to the union of $G_{i} \subseteq \mathbb{Z}_{r} \times S^{1}$. 
Lemma 3.3. For $\delta \in \mathscr{T}$, let $I(\delta)=\left\{i \mid \delta \in G_{i}\right\}$. Then

$$
\mathbb{C}^{(\delta)}=\left\{z \in \mathbb{C}^{n} \mid z_{j}=0 \Longleftrightarrow j \notin I(\delta)\right\},
$$

and

$$
\mathrm{P}_{\mathfrak{a}}^{\mathfrak{b}}(\delta)=S^{(\delta)} \ltimes\left(\mathbb{Z}_{r} \times S^{1}\right)
$$

which is a weighted projective space spanned by $\mathrm{p}_{i}, i \in I(\delta)$.

Lemma 3.4. Suppose that

$$
\delta=\left(e^{-2 \pi i \frac{b}{r}}, e^{2 \pi i R}\right) .
$$

The degree shifting of $\delta$ in $\mathrm{P}_{\mathfrak{a}}^{\mathfrak{b}}$ (cf. [11]) is

$$
\operatorname{deg} \operatorname{sh}(\delta)=\sum_{u \notin I(\delta)}\left\{-\frac{b}{r} \beta_{u}+\alpha_{u} R\right\}=\sum_{u}\left\{-\frac{b}{r} \beta_{u}+\alpha_{u} R\right\}
$$

Here $\{c\}:=c-[c]$ is the fractional part of $c$.

Proof. For $1 \leq u \leq n, \delta$ acts on $z_{u}$ via

$$
\delta z_{u}=e^{-2 \pi i \frac{b \beta_{u}}{r}} e^{2 \pi i \alpha_{u} R} z_{u}
$$

In particular when $u \in I(\delta), \delta z_{u}=z_{u}$, that is $-\frac{b}{r} \beta_{u}+\alpha_{u} R \in \mathbb{Z}$. Then by the definition of degree shifting, the formula follows.

Remark 3.5. In later computation, we will use the case that

$$
\delta=\left(\zeta_{r}^{-1}, e^{2 \pi i R}\right)
$$

Define

$$
\tau(R, u):=-\frac{\beta_{u}}{r}+\alpha_{u} R
$$

Then

$$
\operatorname{deg} \operatorname{sh}(\delta)=\sum_{u}\{\tau(R, u)\}
$$

3.2. Weighted blowups of vector bundles. Now let $\pi: E \rightarrow B$ be a rank $n$ complex vector bundle. Suppose that $P$ is the $K$-principal bundle of $E$, i.e,

$$
\mathrm{E}=\mathrm{P} \times{ }_{K} \mathbb{C}^{n}
$$

where $\mathrm{P}$ is a $K$-principal bundle. Let $S_{\mathfrak{a}}^{1}$ be an action on $\mathbb{C}^{n}$ that commutes with $K$-action. Then we make the following definitions:

(1) the weight-a projectivization of $\mathrm{E}$ with respect to the $S_{\mathfrak{a}}^{1}$ action:

$$
P E_{\mathfrak{a}}=P \times_{K} P,
$$

(2) the weight-a blowup of E along B with respect to the $S_{\mathfrak{a}}^{1}$ action:

$$
\underline{\mathrm{E}}_{\mathfrak{a}}=\mathrm{P} \times{ }_{K}\left[\underline{\mathbb{C}}^{n}\right]_{\mathfrak{a}}
$$

(3) the weight-a projectification of E with respect to the $S_{\mathfrak{a}}^{1}$ action:

$$
\overline{\mathrm{E}}_{\mathfrak{a}}=\mathrm{P} \times_{K}\left[\overline{\mathbb{C}^{n}}\right]_{\mathfrak{a}} .
$$


There are natural morphisms from $P E_{\mathfrak{a}}, \bar{E}_{\mathfrak{a}}$ and $\underline{E}_{\mathfrak{a}}$ to $B$ induced from $\pi: E \rightarrow B$, we denote them all by $\pi$ and call them projections. There is a natural morphism $\kappa: \underline{E}_{\mathfrak{a}} \rightarrow E$ induced by $\kappa:\left[\underline{\mathbb{C}^{n}}\right]_{\mathfrak{a}} \rightarrow \mathbb{C}^{n}$ (cf. (3.1)), which we still denote by $\kappa$. Then

$$
\kappa^{-1}(B) \cong P E_{\mathfrak{a}}
$$

is the exceptional divisor of the weight-a blowup. There is an infinity divisor $P E_{\mathfrak{a}}$ in $\bar{E}_{\mathfrak{a}}$ and

$$
\bar{E}_{\mathfrak{a}} \backslash P E_{\mathfrak{a}}=E .
$$

We now describe some relations between $P E_{\mathfrak{a}}$ and $\mathrm{B}$.

First of all, as in the smooth case, we still have

$$
H^{*}\left(\mathrm{PE}_{\mathfrak{a}}\right) \cong H^{*}(\mathrm{~B})\left\{1, H, \ldots, H^{n-1}\right\}
$$

where $H$ is the first Chern class of tautological line bundle of $P E_{\mathfrak{a}}$.

Second, the inertia spaces of them have the following relation.

Lemma 3.6. The projection $\pi: \mathrm{PE}_{\mathfrak{a}} \rightarrow \mathrm{B}$ induces a morphism between their inertia spaces:

$$
\mathrm{I} \pi: \mathrm{IPE}_{\mathfrak{a}} \rightarrow \mathrm{IB} .
$$

In particular, it induces a map on $\mathscr{T}$ :

$$
\pi_{\mathscr{T}}: \mathscr{T}^{\mathrm{PE}} \rightarrow \mathscr{T}^{\mathrm{B}}
$$

When restricting on twisted sectors,

$$
\mathrm{I} \pi: \mathrm{PE}_{\mathfrak{a}}(\delta) \rightarrow \mathrm{B}\left(\pi_{\mathscr{T}}(\delta)\right)
$$

is a projectivization of certain vector bundle.

Proof. We give an explicit description of morphisms by using local coordinate charts.

Let $U \rtimes G_{x}$ be a local orbifold chart for $\mathbf{U} \subseteq|\mathrm{B}|$ such that the bundle $\mathrm{E}$ over $\mathbf{U}$ can be trivialized as

$$
\left.\mathrm{E}\right|_{\mathrm{U}} \cong G_{x} \ltimes\left(U \times \mathbb{C}^{n}\right)
$$

Then

$$
\left.P E_{\mathfrak{a}}\right|_{\mathrm{U}} \cong\left(G_{x} \times S_{\mathfrak{a}}^{1}\right) \ltimes\left(U \times S^{2 n-1}\right)
$$

Locally,

(1) a twisted sector of $B$ is indexed by a conjugate class $(k), k \in G_{x}$, and is $C_{G_{x}}(k) \ltimes U^{k}$;

(2) a twisted sector of $P E_{\mathfrak{a}}$ is indexed by a conjugate class $(\delta)$,

$$
\delta=(k, t) \in G_{x} \times S^{1},
$$

and is a fibration

$$
\mid \pi:\left(C_{G_{x}}(k) \times S^{1}\right) \ltimes\left(U \times S^{2 n-1}\right)^{\delta} \rightarrow C_{G_{x}}(k) \ltimes U^{k} .
$$

which is the projectivization of

$$
C_{G_{x}}(k) \ltimes\left(U \times \mathbb{C}^{n}\right)^{\delta} \rightarrow C_{G_{x}}(k) \ltimes U^{k} .
$$

Set $\pi_{\mathscr{T}}(\delta)=(k)$. This gives us the map $\pi_{\mathscr{T}}$. 
3.3. Weighted blowups of $X$ along $S$. Suppose that the real codimension of $S$ is $2 n$. Let $N$ be the normal bundle of $S$. Then the weight-a blowup of $N$ induces a weight-a blowup of $X$ along $S$, we denote the resulting orbifold groupoid by $\underline{X}_{\mathfrak{a}}$.

As in the smooth case, $X$ then degenerates into two components:

$$
X^{-}=\underline{X}_{\mathfrak{a}}, \quad X^{+}=\bar{N}_{\mathfrak{a}} .
$$

They have a common divisor $Z=P N_{\mathfrak{a}}$. We write

$$
X \stackrel{\text { degenerate }}{\longrightarrow} X^{-} \wedge_{\mathrm{PN}_{\mathfrak{a}}} \mathrm{X}^{+}, \quad(\mathrm{X}, \mathrm{S}) \stackrel{\text { degenerate }}{\longrightarrow} \mathrm{X}^{-} \wedge_{\mathrm{PN}_{\mathfrak{a}}}\left(\mathrm{X}^{+}, \mathrm{S}\right) .
$$

There is also a natural projection $\kappa: \underline{X}_{\mathfrak{a}} \rightarrow X$ obtained from $\kappa: \bar{N}_{\mathfrak{a}} \rightarrow N$. Hence there is

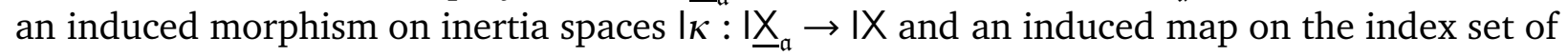
twisted sectors as Lemma 3.6:

$$
\kappa_{\mathscr{T}}: \mathfrak{T}^{\mathrm{X}}{ }_{\mathfrak{a}} \rightarrow \mathscr{T}^{\mathrm{X}}
$$

$\kappa$ is an isomorphism out of a neighborhood of $Z$ and of $S$.

For a twisted sector $X(t)$ of $X$,

- if $X(t) \cap I S=\varnothing$, after blowing up it becomes a twisted sector of $\underline{X}_{\mathfrak{a}}$, which we denote by $\underline{X}_{\mathfrak{a}}(t)$.

- if $\bar{X}(t) \cap I S \neq \varnothing$, after blowing up it lifts to several twisted sectors of $\underline{X}_{\mathfrak{a}}$ with one of them corresponding to $\delta=(k, 1)$ in (3.9); we also denote this one by $\underline{\mathrm{X}}_{\mathfrak{a}}^{a}(t)$.

For both cases, we call $\underline{X}_{\mathfrak{a}}(t)$ the direct transformation of $X(t)$. We denote the set of indices of this kind of twisted sectors of $\underline{X}_{\mathfrak{a}}$ by $\mathscr{T}_{1}^{\underline{X}_{\mathfrak{a}}}$. With these notation, $\kappa_{\mathscr{T}}$ restricts to a bijection

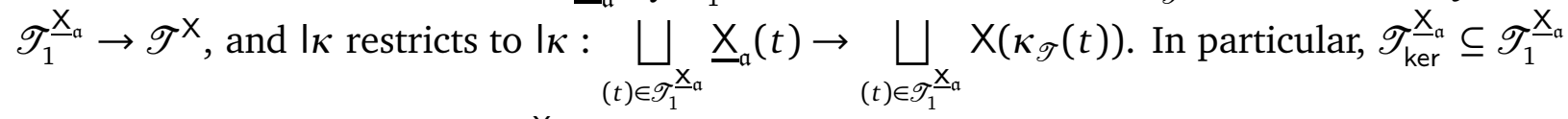
and $\kappa_{\mathscr{T}}$ matches it up with $\mathscr{T}_{\mathrm{ker}}^{\mathrm{X}}$. Therefore $I_{\kappa}$ maps $\operatorname{ker} \underline{X}_{\mathfrak{a}}$ to $\mathrm{ker} X$, and matches up their connected components.

\section{ORBIFOLD GROMOV-WitTEN THEORY}

In this section, we briefly review the Gromov-Witten theory for orbifolds. We emphasize three types of invariants: absolute Gromov-Witten invariants of a compact symplectic orbifold $X$; relative Gromov-Witten invariants of a relative pair $(X \mid Z)$, where $Z$ is a divisor of $X$; absolute Gromov-Witten invariants of a pair $(X, S)$, where $S$ is a compact symplectic sub-orbifold of $X$. The main purpose of this section is to introduce notations used in this paper.

4.1. Absolute Gromov-Witten invariants. Let $\overline{\mathscr{M}}_{g, A, t}(\mathrm{X})$ be the moduli space of holomorphic curves of the type that

(1) the domain curve is connected and of genus $g$,

(2) there are $k$-marked points each of which is associated with a twisted sector $X\left(t_{i}\right), 1 \leq$ $i \leq k$, and $\mathfrak{t}=\left(t_{1}, \ldots, t_{k}\right)$

(3) the curve represents a homology class $A \in H_{2}(X, \mathbb{Z})$.

Let $\mathrm{A}$ denote the combinatoric topological data $(g, A, \mathfrak{t})$. We may denote the moduli space by $\overline{\mathscr{M}}_{\mathrm{A}}(\mathrm{X})$.

To associate invariants to the moduli space $\overline{\mathscr{M}}_{\mathrm{A}}(\mathrm{X})$, for each marking we associate it an insertion $\tau_{d_{i}} \alpha_{i}$ where $\alpha_{i} \in H^{*}\left(X\left(t_{i}\right)\right)$ and $d_{i} \geq 0$. Denote the insertion data by

$$
I=\left(\tau_{d_{1}} \alpha_{1}, \ldots, \tau_{d_{k}} \alpha_{k}\right) .
$$


The Gromov-Witten invariant is defined to be

$$
\langle\mathbf{A}(I)\rangle=\int_{\overline{\mathscr{M}}_{\mathrm{A}}(X)}^{\mathrm{vir}} \mathscr{F}_{I}, \quad \text { where } \quad \mathscr{F}_{I}=\prod_{i=1}^{k}\left(\psi^{d_{i}} \cup e v_{i}^{*} \alpha_{i}\right) .
$$

When at least one of $d_{i}$ is positive, $\langle\mathbf{A}(I)\rangle$ is called a descendent invariant, otherwise a nondescendent invariant or a primary invariant.

When the degree of $\mathscr{F}_{I}$ matches the virtual dimension of the moduli space, we call $\mathrm{A}(I)$ an admissible data of (absolute) Gromov-Witten invariant. Let $\mathscr{A}(\mathrm{X})$ denote the set of admissible data. It is convention to use $\mathscr{A}^{\bullet}(\mathrm{X})$ when we allow the domain curve to be disconnected.

4.2. Relative Gromov-Witten invariants. Now consider a relative pair $(X \mid Z)$ where $Z$ is a divisor of $X$. Let

$$
\overline{\mathscr{M}}_{g, A, \mathrm{t} \mid \mathrm{r}}(\mathrm{X} \mid \mathrm{Z})
$$

denote the relative moduli space of stable curves, which has following properties:

(1) the domain curve is connected, and of genus $g$;

(2) there are $k$ (absolute) marked points, each of which is associated with a twisted sector $X\left(t_{i}\right)$ of $X$, and $\mathfrak{t}=\left(t_{1}, \ldots, t_{k}\right)$,

(3) there are $h$ relative marked points, each of which is associated with a twisted sector $\mathrm{Z}\left(s_{j}\right)$ of $\mathrm{Z}$ and a contact order $\ell_{j}$, and

$$
\mathfrak{r}=\left(\left(s_{1}, \ell_{1}\right), \ldots,\left(s_{h}, \ell_{h}\right)\right),
$$

(4) the curve represents a homology class $A \in H_{2}(X, \mathbb{Z})$.

Let $\mathbf{R}$ denote the combinatoric topological data $(g, A, \mathfrak{t} \mid \mathfrak{r})$. We denote the moduli space by $\overline{\mathscr{M}}_{\mathrm{R}}(\mathrm{X} \mid \mathrm{Z})$.

Now for each marking, we associate it an insertion:

- for $i$-th absolute marking, an insertion $\tau_{d_{i}} \alpha_{i}$, where $\alpha_{i} \in H^{*}\left(X\left(t_{i}\right)\right)$;

- for $j$-th relative marking, an insertion $\beta_{j} \in H^{*}\left(Z\left(s_{i}\right)\right)$.

Let

$$
I=\left(\tau_{d_{1}} \alpha_{1}, \ldots, \tau_{d_{k}} \alpha_{k}\right), \quad J_{Z}=\left(\beta_{1}, \ldots, \beta_{h}\right)
$$

be the collection of absolute insertions and relative insertions.

A relative Gromov-Witten invariant is defined to be

$$
\left\langle\mathbf{R}\left(I \mid J_{\mathrm{Z}}\right)\right\rangle=\int_{\overline{\mathscr{M}}_{\mathrm{R}}(\mathrm{X} \mid \mathrm{Z})}^{\mathrm{vir}} \mathscr{F}_{I} \cup \mathscr{G}_{J_{\mathrm{Z}}}
$$

where

$$
\mathscr{F}_{I}=\prod_{i=1}^{k}\left(\psi_{i}^{d_{i}} \cup e v_{i}^{*} \alpha_{i}\right), \quad \mathscr{G}_{J_{\mathbf{Z}}}=\prod_{j=1}^{h}\left(r e v_{j}^{*} \beta_{j}\right),
$$

and $\operatorname{rev}_{j}: \overline{\mathscr{M}}_{\mathbf{R}}(\mathrm{X} \mid \mathrm{Z}) \longrightarrow \mathrm{Z}$ denotes the $j$-th relative evaluation map. Again we call $\mathbf{R}\left(I \mid J_{\mathrm{Z}}\right)$ an admissible data of relative Gromov-Witten invariant if the total degree of $\mathscr{F}_{I}$ and $\mathscr{G}_{J_{\mathbf{Z}}}$ matches the virtual dimension of the moduli space. Let

$$
\mathscr{R}(\mathrm{X} \mid \mathrm{Z}), \quad\left(\mathscr{R}^{\bullet}(\mathrm{X} \mid \mathrm{Z}) \text { resp. }\right)
$$

be the collections of admissible data of relative Gromov-Witten invariants. 
Remark 4.1. In the following for an admissible relative data $\mathbf{R}\left(I \mid J_{Z}\right)$ with $\mathbf{R}=(g, A, \mathfrak{t} \mid \mathfrak{r})$ and $\mathfrak{r}=\left(\left(s_{1}, \ell_{1}\right), \ldots,\left(s_{h}, \ell_{h}\right)\right)$, we will assume that $J_{Z}$ denotes not only the relative insertions but also the relative data $\mathfrak{r}$, i.e.

$$
J_{\mathrm{Z}}=\left(\left(s_{1}, \ell_{1}, \beta_{1}\right), \ldots,\left(s_{h}, \ell_{h}, \beta_{h}\right)\right) .
$$

Then we use the following notations

$$
\mathfrak{r}\left(J_{Z}\right):=\mathfrak{r}=\left(\left(s_{1}, \ell_{1}\right), \ldots,\left(s_{h}, \ell_{h}\right)\right), \quad \mathfrak{i}\left(J_{Z}\right)=\left(\beta_{1}, \ldots, \beta_{h}\right) .
$$

Remark 4.2. Let $\Sigma$ be a basis of $H_{C R}^{*}(Z)$. If the relative insertion $\beta_{j} \in \Sigma$, we call $\mathbf{R}\left(I \mid J_{Z}\right) a$ $\Sigma$-relative admissible data. Let $\mathscr{R}_{\Sigma}(\mathrm{X} \mid \mathrm{Z})\left(\mathscr{R}_{\Sigma}^{\bullet}(\mathrm{X} \mid \mathrm{Z})\right.$ resp. $)$ denote the set of $\Sigma$-data.

\subsection{The degeneration formula.}

4.3.1. Degenerate absolute invariants. Suppose that $X$ degenerates to a pair of orbifolds $X^{ \pm}$ with a common divisor $Z$. Let $\Sigma^{+}$be a basis of $H_{C R}^{*}(Z)$ and $\Sigma^{-}$be its orbifold Poincaré dual.

Definition 4.3. Let

$$
\mathbf{R}_{ \pm}^{\bullet}\left(I^{ \pm} \mid J_{Z}^{ \pm}\right) \in \mathscr{R}_{\Sigma^{ \pm}}^{\bullet}\left(X^{ \pm} \mid Z\right)
$$

be a pair of admissible relative datum. We say that they form a matched pair if $J_{\mathrm{Z}}^{+}$and $J_{\mathrm{Z}}^{-}$are matched in the following sense: they have same cardinality, and for each $j$,

$$
s_{j}^{+}=\left(s_{j}^{-}\right)^{-1}, \quad \ell_{j}^{+}=\ell_{j}^{-}, \quad \beta_{j}^{+}=\left(\beta_{j}^{-}\right)^{*} .
$$

If it is this case, with the understanding in Remark 4.1 we write

$$
J_{\mathrm{Z}}^{+}=\breve{J}_{\mathrm{Z}}^{-}
$$

Suppose that

$$
\left(\mathbf{R}_{+}^{\bullet}\left(I^{+} \mid J_{Z}^{+}\right), \mathbf{R}_{-}^{\bullet}\left(I^{-} \mid J_{Z}^{-}\right)\right)
$$

is a matched pair. The degeneration theory of Gromov-Witten invariants says that the pair can be glued to an admissible data of an absolute Gromov-Witten invariant $\mathbf{A}^{\bullet}(I)$ where $I=I^{+} \cup I^{-}$. We write

$$
\mathbf{A}^{\bullet}(I)=\mathbf{R}_{+}^{\bullet}\left(I^{+} \mid J_{\mathrm{Z}}^{+}\right) * \mathbf{R}_{-}^{\bullet}\left(I^{-} \mid J_{\mathrm{Z}}^{-}\right) .
$$

Let $\mathscr{D}_{\mathbf{A}^{\bullet}(I)}\left(I^{+}, I^{-}\right)$denote the collection of matched pairs $\left(\mathbf{R}_{ \pm}^{\bullet}\left(I^{ \pm} \mid J_{Z}^{ \pm}\right)\right)$such that (4.2) holds.

Then we have a degeneration formula

$$
\left\langle\mathbf{A}^{\bullet}(I)\right\rangle=\sum_{\Omega \in \mathscr{D}_{\mathbf{A}^{\bullet}(I)}\left(I^{+}, I^{-}\right)} c_{\Omega} \cdot\left\langle\mathbf{R}_{-}^{\bullet}\left(I^{-} \mid J_{\mathrm{Z}}^{-}\right)\right\rangle^{\mathrm{X}^{-} \mid \mathrm{Z}} \cdot\left\langle\mathbf{R}_{+}^{\bullet}\left(I^{+} \mid J_{\mathrm{Z}}^{+}\right)\right\rangle^{\mathrm{X}^{+} \mid \mathrm{Z}} .
$$

where $\Omega=\left(\mathbf{R}_{-}^{\bullet}\left(I^{-} \mid J_{Z}^{-}\right), \mathbf{R}_{+}^{\bullet}\left(I^{+} \mid J_{Z}^{+}\right)\right)$, and $c_{\Omega}$ is a constant depends on $\mathfrak{r}_{ \pm}$in $\mathbf{R}_{ \pm}^{\bullet}$. It would happen that one of the admissible relative data in $\Omega=\left(\mathbf{R}_{-}^{\bullet}\left(I^{-} \mid J_{Z}^{-}\right), \mathbf{R}_{+}^{\bullet}\left(I^{+} \mid J_{Z}^{+}\right)\right)$is empty, i.e., $\mathbf{R}_{-}^{\bullet}\left(I^{-} \mid J_{Z}^{-}\right)=\varnothing$, or $\mathbf{R}_{+}^{\bullet}\left(I^{+} \mid J_{Z}^{+}\right)=\varnothing$, then the coefficient $c_{\Omega}=1$, and the invariant for the empty relative data is also set to be 1 . 
4.3.2. Degenerate relative invariants. We also have the degeneration formula for relative orbifold Gromov-Witten invariants. We consider a special case which we will use latter. Let (X|Z) be a relative pair and $\mathbf{R}^{\bullet}(I \mid J)$ be an admissible relative data. We blow up $(X \mid Z)$ along Z with trivial weight $\mathfrak{a}=(1)$ to degenerate $(X \mid Z)$ in to

$$
(X \mid Z) \stackrel{\text { degenerate }}{\longrightarrow}\left(X^{-} \mid Z^{-}\right) \wedge_{Z}\left(Z^{+}\left|X^{+}\right| Z\right)=(X \mid Z) \wedge_{Z}\left(Z_{\infty}|\bar{N}| Z_{0}\right)
$$

where $\bar{N}$ is trivial weight projectification of the normal bundle of $Z$ in $X$ and $Z_{0}, Z_{\infty}$ are the zero and infinity sections. Then we have

$$
\left\langle\mathbf{R}^{\bullet}(I \mid J)\right\rangle^{\mathrm{X} \mid \mathrm{Z}}=\sum_{\Omega \in \mathscr{D}_{\mathbf{R}^{\bullet}(I \mid J)}\left(I^{+}, I^{-}\right)} c_{\Omega} \cdot\left\langle\mathbf{R}_{-}^{\bullet}\left(I^{-} \mid J_{\mathrm{Z}}^{-}\right)\right\rangle^{\mathrm{X} \mid \mathrm{Z}} \cdot\left\langle\mathbf{R}_{+}^{\bullet}\left(J_{Z}^{+}\left|I^{+}\right| J\right)\right\rangle^{\mathrm{Z}_{\infty}|\overline{\mathrm{N}}| \mathrm{Z}_{0}}
$$

where $\mathscr{D}_{\mathbf{R}^{\bullet}(I \mid J)}\left(I^{+}, I^{-}\right)$denote the collection of matched pairs

$$
\Omega=\left(\mathbf{R}_{-}^{\bullet}\left(I^{-} \mid J_{\mathrm{Z}}^{-}\right), \mathbf{R}_{+}^{\bullet}\left(J_{\mathrm{Z}}^{+}\left|I^{+}\right| J\right)\right)
$$

(they match along $\mathrm{Z} \in \mathrm{X}$ and $\mathrm{Z}_{\infty} \in \mathrm{X}^{+}$like Definition 4.3, then $J_{\mathrm{Z}}^{-}=\breve{J}_{\mathrm{Z}}^{+}$), such that

$$
\mathbf{R}^{\bullet}(I \mid J)=\mathbf{R}_{-}^{\bullet}\left(I^{-} \mid J_{\mathrm{Z}}^{-}\right) * \mathbf{R}_{+}^{\bullet}\left(J_{\mathrm{Z}}^{+}\left|I^{+}\right| J\right) .
$$

We can also consider nontrivial weight degeneration of $\mathrm{X}$ along $\mathrm{Z}$.

By the Gromov compactness theorem, for a fixed absolute (resp. relative) data $\mathbf{A}^{\bullet}(I)$ (resp. $\mathbf{R}^{\bullet}(I \mid J)$ ) the set $\mathscr{D}_{\mathbf{A}^{\bullet}(I)}\left(I^{+}, I^{-}\right)$(resp. $\mathscr{D}_{\mathbf{R}^{\bullet}(I \mid J)}\left(I^{+}, I^{-}\right)$) is a finite set.

4.4. Absolute Gromov-Witten invariants of symplectic pairs. Let $S$ be a compact symplectic sub-orbifold of $\mathrm{X}$. A cohomology class $\alpha \in H_{C R}^{*}(\mathrm{X})$ is called to be supported on $\mathrm{S}$ if it is of the form $\theta \cup[\mathrm{IS}]$, where $\theta \in H_{C R}^{*}(\mathrm{~S})$ and [IS] denotes the Thom class of IS in IX. If we fix a basis $\sigma$ of $H_{C R}^{*}(\mathrm{~S})$ and let $\sigma^{*}$ be its dual, we say $\theta \cup[\mathrm{IS}]$ is a $\sigma$-class if $\theta \in \sigma$.

Definition 4.4. We call an admissible data $\mathrm{A}(I)$ of absolute Gromov-Witten invariant is a data relative to $S$ if, for each insertion $\tau_{d_{i}} \alpha_{i}, d_{i}>0$ only when $\alpha_{i}$ is supported on $S$. We call these insertions to be S-supported insertions. $\mathbf{A}(I)$ is called a $\sigma$-data relative to $\mathrm{S}$ if those class $\alpha_{i}$ supported on $\mathrm{S}$ are $\sigma$-classes.

We separate I into two parts: let $I_{S}$ denote the collection of S-supported insertions and $I^{\prime}$ denote the rest. We write $\mathrm{A}(I)$ to be $\mathrm{A}\left(I^{\prime} ; I_{\mathrm{S}}\right)$. Let $\mathscr{A}^{\bullet}(\mathrm{X}, \mathrm{S})$ (resp. $\mathscr{A}_{\sigma}^{\bullet}(\mathrm{X}, \mathrm{S})$ ) denote the set of (resp. $\sigma_{-}$) admissible data of absolute invariants relative to $\mathrm{S}$.

We may apply the degeneration formula to $\left\langle\mathbf{A}\left(I ; I_{\mathrm{S}}\right)\right\rangle$ for the degeneration

$$
(X ; S) \stackrel{\text { degenerate }}{\longrightarrow} X^{-} \wedge_{Z}\left(X^{+}, S\right)=\underline{X}_{\mathfrak{a}} \wedge_{Z}\left(\bar{N}_{\mathfrak{a}}, S\right),
$$

then we have

$$
\left\langle\mathbf{A}\left(I ; I_{\mathrm{S}}\right)\right\rangle=\sum_{\Omega \in \mathscr{D}_{\mathrm{A}\left(I ; I_{\mathrm{S}}\right)}\left(I^{+}, I^{-}\right)} c_{\Omega} \cdot\left\langle\mathbf{R}^{-}\left(I^{-} \mid J_{\mathrm{Z}}^{-}\right)\right\rangle^{\underline{\mathbf{X}}_{\mathfrak{a}} \mid \mathrm{Z}} \cdot\left\langle\mathbf{R}^{+}\left(I^{+} ; I_{\mathrm{S}} \mid J_{\mathrm{Z}}^{+}\right)\right\rangle^{\overline{\mathrm{N}}_{\mathfrak{a}} \mid \mathrm{Z}},
$$

where $\Omega=\left(\mathbf{R}_{-}\left(I^{-} \mid J_{\mathrm{Z}}^{-}\right), \mathbf{R}_{+}\left(I^{+} ; I_{\mathrm{S}} \mid J_{\mathrm{Z}}^{+}\right)\right)$and $J_{\mathrm{Z}}^{-}=\check{J}_{\mathrm{Z}}^{+}$. 


\section{Certain invariants of $\left(\overline{\mathrm{E}}_{\mathfrak{a}} \mid \mathrm{PE} \mathrm{E}_{\mathfrak{a}}\right)$} E.

In this section we study certain relative invariants of $\left(\bar{E}_{\mathfrak{a}} \mid P E_{\mathfrak{a}}\right)$. We treat $B$ as the 0 -section

Description of topological data $\mathbf{R}$ and insertions:

(1) The domain curve $S^{2}$ is the orbifold sphere with 2 orbifold points at 0 and $\infty$. Be precise, there are two orbifold charts

$$
\mathrm{C}_{0}=\mathbb{Z}_{r} \ltimes \mathbb{C}, \quad \mathrm{C}_{\infty}=\mathbb{Z}_{h} \ltimes \mathbb{C},
$$

respectively. Set

$$
\mathrm{q}_{0}=\mathbb{Z}_{r} \ltimes\{0\}, \quad \mathrm{q}_{\infty}=\mathbb{Z}_{h} \ltimes\{\infty\} .
$$

(2) Marked points: $q_{0}$ is an absolute marked point and $q_{\infty}$ is a relative marked point.

(3) The insertion for $q_{0}$ is supported in $B: \tau_{c}(\theta \cup[I B])$, where $\theta \in H^{*}(B(t))$ and [IB] is the Thom form of $\mathrm{B}(t)$ in $\mathrm{E}(t)$.

(4) The insertion for $q_{\infty}$ is $\beta \in H^{*}\left(\operatorname{PE}_{\mathfrak{a}}(s)\right)$; suppose that $\ell$ is the contact order at $q_{\infty}$.

(5) $A \in H_{2}\left(\left|\overline{\mathrm{E}}_{\mathfrak{a}}\right|\right)$ is of fiber class, i.e, $\pi_{*}(A)=0$.

Then

$$
\mathbf{R}=(g=0, A, \mathfrak{t}=(t) \mid \mathfrak{r}=(s, \ell)), \quad I_{\mathrm{B}}=\left(\tau_{c}(\theta \cup[\mathrm{B}(t)])\right), \quad J_{\mathrm{PE}_{\mathfrak{a}}}=(\beta) .
$$

Let $\overline{\mathscr{M}}_{\mathrm{R}}\left(\overline{\mathrm{E}}_{\mathfrak{a}} \mid \mathrm{PE} \mathrm{E}_{\mathfrak{a}}\right)$ be the moduli space. The top stratum of the moduli space is

$$
\mathscr{M}_{\mathrm{R}}\left(\overline{\mathrm{E}}_{\mathfrak{a}} \mid \mathrm{PE} \mathrm{E}_{\mathfrak{a}}\right)=\frac{\mathrm{Hol}_{\mathrm{R}}\left(\overline{\mathrm{E}}_{\mathfrak{a}} \mid \mathrm{PE} \mathrm{E}_{\mathfrak{a}}\right)}{\operatorname{Aut}\left(\mathrm{S}^{2} \backslash\left\{\mathrm{q}_{0}, \mathrm{q}_{\infty}\right\}\right)} .
$$

Here $\mathrm{Hol}_{\mathrm{R}}\left(\bar{E}_{\mathfrak{a}} \mid P E_{\mathfrak{a}}\right) \subseteq \operatorname{Mor}\left(\bar{E}_{\mathfrak{a}} \mid P E_{\mathfrak{a}}\right)$ consists of holomorphic morphisms. Usually, $\operatorname{Hol}_{\mathbf{R}}\left(\bar{E}_{\mathfrak{a}} \mid P E_{\mathfrak{a}}\right)$ is denoted by $\widetilde{\mathscr{M}}_{\mathrm{R}}\left(\overline{\mathrm{E}}_{\mathfrak{a}} \mid P E_{\mathfrak{a}}\right)$. Here, we use the new notation to emphasize its groupoid structure.

Remark 5.1. Suppose that $\mathrm{u}: \mathrm{S}^{2} \rightarrow \overline{\mathrm{E}}_{\mathfrak{a}}$ is a holomorphic morphism in $\operatorname{Hol}_{\mathrm{R}}^{0}\left(\overline{\mathrm{E}}_{\mathfrak{a}} \mid \mathrm{PE} \mathrm{a}_{\mathfrak{a}}\right)$. Then

(1) the morphism covers a fiber, i.e, for $S^{2} \stackrel{u}{\rightarrow} \bar{E}_{\mathfrak{a}} \stackrel{\pi}{\rightarrow} B$, the induced map $|\pi \circ u|:\left|S^{2}\right| \rightarrow|B|$ on coarse spaces is constant;

(2) u maps $\mathrm{C}_{0}$ to $\mathrm{E}$;

(3) $\mathrm{u}: \mathrm{q}_{0} \rightarrow \mathrm{E}(t)$ and $\mathrm{u}: \mathrm{q}_{\infty} \rightarrow \mathrm{PE}_{\mathfrak{a}}(s)$.

The goal in this section is to understand invariants

$$
\left\langle\mathbf{R}\left(I_{\mathrm{B}} \mid J_{\mathrm{PE}_{\mathfrak{a}}}\right)\right\rangle
$$

under certain conditions.

We first give a completely understanding of invariants when $E=\mathbb{Z}_{r}^{\mathfrak{b}} \ltimes \mathbb{C}^{n}$, an orbifold bundle over $B_{r}$. This is explained in $\S 5.1-\S 5.2$. Then in $\S 5.3$ and $\S 5.4$ we reduce the study of general cases to this special case when $\mathbf{R}\left(I_{\mathrm{B}} \mid J_{\mathrm{PE}_{\mathfrak{a}}}\right)$ is under certain conditions.

5.1. Special case: $E=\mathbb{Z}_{r}^{\mathfrak{b}} \ltimes \mathbb{C}^{n}$. We start with this special case. Then

$$
\bar{E}_{\mathfrak{a}}=\left[\overline{\mathbb{C}^{n}}\right]_{\mathfrak{a}}^{\mathfrak{b}}=P_{(\mathfrak{a}, 1)}^{(\mathfrak{b}, 0)}, \quad P E_{\mathfrak{a}}^{\mathfrak{b}}=P_{\mathfrak{a}}^{\mathfrak{b}} .
$$

By the definition of the moduli space,

$$
\mathscr{M}_{\mathrm{R}}\left(\left[\overline{\mathbb{C}^{n}}\right]_{\mathfrak{a}}^{\mathfrak{b}} \mid P_{\mathfrak{a}}^{\mathfrak{b}}\right)=\frac{\operatorname{Hol}_{\mathrm{R}}\left(\mathrm{S}^{2},\left[\overline{\mathbb{C}^{n}}\right]_{\mathfrak{a}}^{\mathfrak{b}}\right)}{\operatorname{Aut}\left(\mathrm{S}^{2} \backslash\left\{\mathrm{q}_{0}, \mathrm{q}_{\infty}\right\}\right)}
$$

Without loss of generality, we make the following assumption. 
Assumption 5.2. The twisted sector $t$ associated to $\mathrm{q}_{0}$ is $\zeta_{r}=\exp ^{2 \pi i \frac{1}{r}} \in \mathbb{Z}_{r}$.

The invariant to compute is

$$
\left\langle\mathbf{R}\left(\tau_{c}\left(\Theta_{(t)}\right) \mid H_{(s)}^{d}\right)\right\rangle=\int_{\overline{\mathscr{M}}_{\mathbf{R}}} \psi^{c} \cup \mathrm{ev}_{0}{ }^{*} \Theta_{t} \cup \operatorname{rev}_{\infty}^{*} H_{(s)}^{d},
$$

where $\Theta_{(t)}$ is the Thom class of $\mathrm{E}(t) \rightarrow \mathrm{B}(t)$ and $H_{(s)}$ is the generator of $H^{*}\left(\mathrm{P}_{\mathfrak{a}}^{\mathfrak{b}}(s)\right)$. The following theorem is the main theorem of the section, and is the most important technical result for this paper.

Theorem 5.3. Suppose the data $\mathbf{R}\left(\tau_{c}\left(\Theta_{(t)}\right) \mid H_{(s)}^{d}\right)$ is admissible and satisfies Assumption 5.2. Then

(1) c can be any nonnegative integer,

(2) $\mathbf{R}$ and $d$ are uniquely determined by $c$,

(3) the invariant $\left\langle\mathbf{R}\left(\tau_{c}\left(\Theta_{(t)}\right) \mid H_{(s)}^{d}\right)\right\rangle$ is non-zero.

From now on, in the rest of subsection we write $\mathscr{M}_{\mathrm{R}}$ for top strata of the moduli space.

5.1.1. Description of $\mathscr{M}_{\mathrm{R}}$. We want to give a more explicit description of the right hand side of (5.3). This consists of two steps.

Step 1. Compare $\mathrm{Hol}_{\mathbf{R}}\left(\mathrm{S}^{2},\left[\overline{\mathbb{C}^{n}}\right]_{\mathfrak{a}}^{\mathfrak{b}}\right)$ with $\mathrm{Hol}\left(\mathrm{C}_{0}, \mathbb{Z}_{r}^{\mathfrak{b}}\right) \ltimes \mathbb{C}^{n}$. The restriction of morphisms from $\mathrm{S}^{2}$ to $\mathrm{C}_{0}$ induces a groupoid morphism

$$
\Psi: \operatorname{Hol}_{\mathbf{R}}\left(\mathrm{S}^{2},\left[\overline{\mathbb{C}^{n}}\right]_{\mathfrak{a}}^{\mathfrak{b}}\right) \hookrightarrow \operatorname{Hol}\left(\mathrm{S}^{2},\left[\overline{\mathbb{C}^{n}}\right]_{\mathfrak{a}}^{\mathfrak{b}}\right) \stackrel{\mathrm{I}_{0}}{\longrightarrow} \operatorname{Hol}\left(\mathrm{C}_{0},\left[\overline{\mathbb{C}^{n}}\right]_{\mathfrak{a}}^{\mathfrak{b}}\right) \longrightarrow \operatorname{Hol}\left(\mathrm{C}_{0}, \mathbb{Z}_{r}^{\mathfrak{b}} \ltimes \mathbb{C}^{n}\right) .
$$

The last arrow follows from the fact that $u: C_{0} \rightarrow E$ when $u \in \mathrm{Hol}_{R}$.

Conversely, for

$$
v \in \operatorname{Hol}^{0}\left(C_{0}, \mathbb{Z}_{r}^{\mathfrak{b}} \ltimes \mathbb{C}^{n}\right)
$$

(with finite energy), by the removable singularity theorem, $v$ can be extended to define a morphism

$$
\Phi^{0}(\mathrm{v}):=\overline{\mathrm{v}}: \mathrm{S}^{2} \rightarrow\left[\overline{\mathbb{C}^{n}}\right]_{\mathfrak{a}}^{\mathfrak{b}} .
$$

We say that $v$ is of R-type if $\bar{v} \in \operatorname{Hol}_{R}^{0}\left(S^{2},\left[\overline{\mathbb{C}^{n}}\right]_{\mathfrak{a}}^{\mathfrak{b}}\right)$. Let

$$
\operatorname{Hol}_{\mathbf{R}}\left(\mathrm{C}_{0}, \mathbb{Z}_{r}^{\mathfrak{b}} \ltimes \mathbb{C}^{n}\right) \subseteq \operatorname{Hol}\left(\mathrm{C}_{0}, \mathbb{Z}_{r}^{\mathfrak{b}} \ltimes \mathbb{C}^{n}\right)
$$

be the space of morphisms of R-type. Then removable singularity theorem for orbifold holomorphic morphisms says that

$$
\Phi: \mathrm{Hol}_{\mathbf{R}}\left(\mathrm{C}_{0}, \mathbb{Z}_{r}^{\mathfrak{b}} \ltimes \mathbb{C}^{n}\right) \rightarrow \mathrm{Hol}_{\mathbf{R}}\left(\mathrm{S}^{2},\left[\overline{\mathbb{C}^{n}}\right]_{\mathfrak{a}}^{\mathfrak{b}}\right)
$$

is an equivalence, which is the inverse of $\Psi$. On the other hand,

Lemma 5.4. $\mathrm{Hol}_{\mathbf{R}}\left(\mathrm{C}_{0}, \mathbb{Z}_{r}^{\mathfrak{b}} \ltimes \mathbb{C}^{n}\right)=\mathbb{Z}_{r} \ltimes\left(\operatorname{Hol}_{\mathrm{R}}^{0}\left(\mathrm{C}_{0}, \mathbb{Z}_{r}^{\mathfrak{b}} \ltimes \mathbb{C}^{n}\right)\right)$.

Proof. For any $\mathrm{u} \in \mathrm{Hol}^{0}$, the arrows from $\mathrm{u}$ are identified with constant maps from $\mathbb{C}$ to $\mathbb{Z}_{r}$. Hence $\operatorname{Hom}(\mathrm{u}, \cdot) \cong \mathbb{Z}_{r}$. For $\xi \in \mathbb{Z}_{r}$,

$$
(\xi \cdot u)^{0}=\xi \cdot u^{0},(\xi \cdot u)^{1}=u^{1} .
$$

Hence $\mathrm{Hol}^{1}$ acts on $\mathrm{Hol}^{0}$ as a $\mathbb{Z}_{r}$-action. 
Step 2, treatment of automorphism groups. For $\mathrm{C}_{0}=\mathbb{Z}_{r} \ltimes \mathbb{C}$ we write

$$
\mathrm{C}_{0}=\mathbb{Z}_{r} \ltimes \mathbb{C}_{o}, \quad \mathrm{C}_{0}^{*}=\mathbb{Z}_{r} \ltimes \mathbb{C}_{o}^{*} \cong \mathbb{C}^{*} .
$$

We have an exact sequence

$$
1 \longrightarrow \mathbb{Z}_{r} \longrightarrow \mathbb{C}_{o}^{*} \longrightarrow \mathbb{C}^{*} \longrightarrow 1
$$

On the other hand,

$$
\mathbb{C}_{o}^{*}=\operatorname{Aut}\left(\mathbb{C}_{o}^{*}\right), \quad \mathbb{C}^{*}=\operatorname{Aut}\left(\mathbb{C}^{*}\right)=\operatorname{Aut}\left(S^{2} \backslash\left\{\mathrm{q}_{0}, \mathrm{q}_{\infty}\right\}\right) .
$$

Hence,

$$
1 \longrightarrow \mathbb{Z}_{r} \longrightarrow \operatorname{Aut}\left(\mathbb{C}_{o}^{*}\right) \longrightarrow \operatorname{Aut}\left(S^{2} \backslash\left\{q_{0}, q_{\infty}\right\}\right) \longrightarrow 1
$$

It is not hard to see that

$$
\left(\operatorname{Hol}_{\mathrm{R}}^{0}\left(\mathrm{C}_{0}, \mathbb{Z}_{r}^{\mathfrak{b}} \ltimes \mathbb{C}^{n}\right) / \mathbb{Z}_{r}\right) / \operatorname{Aut}\left(\mathrm{S}^{2} \backslash\left\{\mathrm{q}_{0}, \mathrm{q}_{\infty}\right\}\right) \cong\left(\operatorname{Hol}_{\mathrm{R}}^{0}\left(\mathrm{C}_{0}, \mathbb{Z}_{r}^{\mathfrak{b}} \ltimes \mathbb{C}^{n}\right)\right) / \operatorname{Aut}\left(\mathbb{C}_{o}^{*}\right) .
$$

We conclude that

Lemma 5.5. Suppose that $\mathrm{C}_{0}=\mathbb{Z}_{r} \ltimes \mathbb{C}_{0}$. Then

$$
\mathscr{M}_{\mathrm{R}} \cong \mathbb{C}_{o}^{*} \ltimes \operatorname{Hol}_{\mathrm{R}}^{0}\left(\mathrm{C}_{0}, \mathbb{Z}_{r}^{\mathfrak{b}} \ltimes \mathbb{C}^{n}\right) \text {. }
$$

5.1.2. $\psi$-class associated to $\mathrm{q}_{0}$. For the marking $\mathrm{q}_{0}$ one may associate a line bundle $\mathscr{L}$ over $\overline{\mathscr{M}}_{\mathrm{R}}$ : for each morphism $\mathrm{u}$ we assign it a complex line which is dual to $\mathbb{C}\left(\cong \mathbb{C}_{o} / \mathbb{Z}_{r}\right)$. Using Lemma 5.5 we may give a concrete expression for $\mathscr{L}$.

Let $\check{\mathbb{C}}_{o}$ be the dual space of $\mathbb{C}_{o}$.

$$
\tilde{\mathscr{L}}:=\frac{\check{\mathbb{C}}_{o} \times\left(\operatorname{Hol}_{\mathrm{R}}^{0}\left(\mathrm{C}_{0}, \mathbb{Z}_{r}^{\mathfrak{b}} \ltimes \mathbb{C}^{n}\right)\right)}{\mathbb{C}_{o}^{*}} \rightarrow \frac{\operatorname{Hol}_{\mathrm{R}}\left(\mathrm{C}_{0}, \mathbb{Z}_{r}^{\mathfrak{b}} \ltimes \mathbb{C}^{n}\right)}{\mathbb{C}_{o}^{*}}=\mathscr{M}_{\mathrm{R}}
$$

is a line bundle. Since $\check{\mathbb{C}} \cong \check{\mathbb{C}}_{o} / \mathbb{Z}_{r}$, we have

$$
\tilde{\mathscr{L}}^{\otimes r} \cong \mathscr{L} .
$$

The psi-class is $\psi=c_{1}(\mathscr{L})$.

5.1.3. Description of $\mathbf{R}$. We may assume that $\mathscr{M}_{\mathbf{R}}$ consists of one of following morphisms

$$
\left\{u[j, m] \mid 1 \leq j \leq n, m \in \mathbb{Z}^{+}\right\} .
$$

Here $u[j, m]: S^{2} \rightarrow\left[\overline{\mathbb{C}}_{\mathfrak{a}}^{b}\right.$ is a representable morphism such that (cf. Assumption 5.2)

$$
\begin{aligned}
& \mathrm{u}[j, m]: \mathrm{C}_{0}=\mathbb{Z}_{r} \ltimes \mathbb{C}_{o} \rightarrow \mathbb{Z}_{r}^{\mathfrak{b}} \ltimes \mathbb{C}^{n} ; \\
& x \mapsto\left(\ldots, 0, z_{j}=x^{m}, 0, \ldots\right), \text { id }: \mathbb{Z}_{r} \rightarrow \mathbb{Z}_{r} .
\end{aligned}
$$

Lemma 5.6. For $\mathrm{u}[j, m]$ we have

(1) $m \equiv \beta_{j}(\bmod r)$;

(2) $\mathrm{u}[j, m]:[\overline{\mathbb{C}}]_{p}^{1} \rightarrow\left[\overline{\mathbb{C}^{n}}\right]_{\mathfrak{a}}^{\mathfrak{b}}$, and

$$
u^{0}[j, m]:[x, y] \mapsto\left[\ldots 0, z_{j}=x^{m}, 0, \ldots, 0, w=y^{q}\right],
$$

where $p, q$ are determined by $p / q=\alpha_{j} / m$ such that $(p, q)=1$,

(3) $\mathrm{u}[j, m]:\left\langle e^{2 \pi i \frac{1}{p r}}\right\rangle \ltimes\left\{q_{\infty}\right\} \mapsto\left\langle\left(e^{-2 \pi i \frac{1}{r}}, e^{2 \pi i \frac{m}{\alpha_{j} r}}\right)\right\rangle \ltimes\left\{p_{j}\right\}$.

Here we identify $\mathrm{S}^{2}$ as $[\overline{\mathbb{C}}]_{p}^{1}$.

Remark 5.7. We make several important remarks:

- orbifold structure of $\mathrm{S}^{2}($ at $\infty)$ is determined by $\left.\mathrm{u}[j, m]\right|_{\mathrm{C}_{0}}$, or by the pair $(j, m)$; 
- the twisted sector s associated to $\mathrm{q}_{\infty}$ given in (3) is determined by $(j, m)$ as well, moreover the twisted sector

$$
\pi_{\mathscr{T}}(s)=\bar{t},
$$

as $\left(e^{-2 \pi i \frac{1}{r}}, e^{2 \pi i \frac{m}{\alpha_{j} r}}\right) \mapsto e^{-2 \pi i \frac{1}{r}}$ (see (2.1) for the definition of ${ }^{-}$on inertia spaces and on the index set $\mathscr{T})$;

- for two different pairs $\left(j_{1}, m_{1}\right)$ and $\left(j_{2}, m_{2}\right)$, the twisted sectors associated to $q_{\infty}$ are the same if and only if

$$
\exp \left(2 \pi i \frac{m_{1}}{\alpha_{j_{1}} r}\right)=\exp \left(2 \pi i \frac{m_{2}}{\alpha_{j_{2}} r}\right) ;
$$

- for two different pairs $\left(j_{1}, m_{1}\right)$ and $\left(j_{2}, m_{2}\right)$, they are in same $\mathscr{M}_{\mathrm{R}}$, i.e, they also represent a same homology class if and only if

$$
\frac{m_{1}}{\alpha_{j_{1}} r}=\frac{m_{2}}{\alpha_{j_{2}} r} .
$$

We conclude that $\mathbf{R}$ is completely determined by the number $\frac{m}{\alpha_{j} r}$. This leads to the following function

$$
\Lambda:[1, n]_{\mathbb{Z}} \times \mathbb{Z}_{\geq 0} \rightarrow \mathbb{Q}, \quad \Lambda(j, a)=\frac{\beta_{j}+a r}{\alpha_{j} r} .
$$

Here $[1, n]_{\mathbb{Z}}=[1, n] \cap \mathbb{Z}$. We write $m$ as $\beta_{j}+$ ar, $a \geq 0$. Set $\mathscr{R}$ be the image of $\Lambda$.

These observations implies the following lemma.

Lemma 5.8. Given $R \in \mathscr{R}$, it determines a topological data $\mathbf{R}$ uniquely by

$$
t=\zeta_{r}, \quad s=\left(\zeta_{r}^{-1}, e^{2 \pi i R}\right), A=R[F],
$$

and contact order $\ell=R$. Conversely, if $\mathscr{M}_{\mathbf{R}}$ is non-empty, $\mathbf{R}$ must be obtained by this way.

Another way to say is that $\mathbf{R}$ is completely determined by the homology class $A$ under Assumption 5.2. From now on we denote the moduli space by $\mathscr{M}_{R}, R \in \mathscr{R}$.

5.1.4. Dimension of $\mathscr{M}_{R}$. In order to compute the virtual dimension of moduli space $\mathscr{M}_{R}, R \in$ $\mathscr{R}$, we introduce two functions (of ranking) $r k^{\circ}$ and $r k_{\circ}$ on $\mathscr{R}$ :

$$
\begin{aligned}
& r k^{\circ}(R):=\sharp\{(j, a) \mid \Lambda(j, a)<R\}+1 ; \\
& r k_{\circ}(R):=\sharp\{(j, a) \mid \Lambda(j, a) \leq R\} .
\end{aligned}
$$

Remark 5.9. We list some facts.

- The dimension of the twisted sector of $s=\left(e^{-2 \pi i \frac{1}{r}}, e^{2 \pi i R}\right)$ is

$$
D_{s}:=\sharp \Lambda^{-1}(R)-1=r k_{\circ}(R)-r k^{\circ}(R) .
$$

- The dimension of the twisted sector of $t$ is

$$
D_{t}:=\sharp\left\{j \mid \beta_{j}=r\right\} .
$$

Theorem 5.10. The virtual dimension of moduli space $\mathscr{M}_{R}$ is

$$
\operatorname{dim}_{\mathbb{C}}\left(\mathscr{M}_{R}\right)=r k_{\circ}(R)-1+D_{t} .
$$

The proof of this theorem is given in §5.1.6. 
5.1.5. Proof of Theorem 5.3: (1) and (2). We assume Theorem 5.10.

First, suppose that $\Lambda$ is injective, then $r k_{\circ}=r k^{\circ}$, which we denote by $r k$. Note that $r k \circ \Lambda$ gives a ranking for pairs $\{(j, a)\}$ and for elements in $\mathscr{R}$. Hence

$$
[1, n]_{\mathbb{Z}} \times \mathbb{Z}_{\geq 0} \stackrel{\Lambda}{\rightarrow} \mathscr{R} \stackrel{r k}{\rightarrow} \mathbb{Z}_{+}
$$

is bijective.

Now given $c \in \mathbb{Z}_{\geq 0}$ we explain the explicit formula for $\mathbf{R}$ (i.e, $R$ ) and $d$ :

- since $D_{s}=0, d$ must be 0 ;

- choose $R=r k^{-1}(c+1)$. In fact, $c$ satisfies the formula

$$
D_{t}+c=\operatorname{dim}_{\mathbb{C}}\left(\mathscr{M}_{R}\right)=r k(R)-1+D_{t},
$$

where we use Theorem 5.10 for the last "=".

This finishes the proof of (1) and (2) when $\Lambda$ is injective.

Now suppose that $\Lambda$ is not injective. Define

$$
\tilde{\mathscr{R}}=\left\{(R, \ell) \in \mathscr{R} \times \mathbb{Z} \mid 0 \leq \ell \leq D_{s}\right\} .
$$

Recall that $D_{s}=\sharp \Lambda^{-1}(R)-1$. One may define a one to one correspondence

$$
\psi: \tilde{\mathscr{R}} \longrightarrow[1, n]_{\mathbb{Z}} \times \mathbb{Z}_{\geq 0}
$$

such that for each $R, \psi$ identifies $\{R\} \times\left[0, D_{s}\right]_{\mathbb{Z}}$ with $\Lambda^{-1}(R)$. Write $\tilde{\Lambda}=\psi^{-1}$. Define

$$
\widetilde{r k}: \tilde{\mathscr{R}} \longrightarrow \mathbb{Z}_{+}, \widetilde{r k}(R, \ell)=r k_{\circ}(R)-\ell \text {. }
$$

Then one can see that

$$
[1, n]_{\mathbb{Z}} \times \mathbb{Z}_{\geq 0} \stackrel{\tilde{\Lambda}}{\rightarrow} \tilde{\mathscr{R}} \stackrel{\widetilde{r k}}{\rightarrow} \mathbb{Z}_{+}
$$

is bijective. Now in Theorem 5.3, $c$ determines $R$ and $d$ by

$$
(R, d)=\widetilde{r k}^{-1}(c+1) \text {. }
$$

5.1.6. Proof of Theorem 5.10. Take a pair $(j, a)$ such that

$$
\Lambda(j, a)=\frac{\beta_{j}+a r}{\alpha_{j} r}=R .
$$

Set $m=\beta_{j}+a r$. Then $u[j, m]$ is in $\mathscr{M}_{R} . \mathrm{u}[j, m]$ maps $\mathrm{q}_{\infty}$ to $\langle s\rangle \ltimes\left\{\mathrm{p}_{j}\right\}$, where

$$
s=\left(e^{-2 \pi i \frac{1}{r}}, e^{2 \pi i R}\right)
$$

The degree shifting of $s$ in $\bar{E}_{\mathfrak{a}}$ is (cf. (3.5) and the definition of $\tau(R, u)$ in (3.7))

$$
\operatorname{degsh}(s)=\sum_{u \neq j}\left\{-\frac{\beta_{u}}{r}+\alpha_{u} R\right\}+\{R\}=\sum_{u}\{\tau(R, u)\}+\{R\} .
$$

Note that $\tau(R, j)=\left(m-\beta_{j}\right) / r=a$, consequently $\{\tau(R, j)\}=0$.

Proposition 5.11. The virtual dimension of $\mathscr{M}_{R}$ is

$$
\operatorname{dim}_{\mathbb{C}}\left(\mathscr{M}_{R}\right)=\sum_{u=1}^{n}[\tau(R, u)]+n-1+D_{t},
$$

where $D_{t}$ is the complex dimension of the twisted sector of $t=e^{-2 \pi i \frac{1}{r}}$. 
Proof. By the formula of the virtual dimension for the relative moduli space, we have

$$
\operatorname{dim}_{\mathbb{C}}\left(\mathscr{M}_{R}\right)=\frac{1+\sum_{u} \alpha_{u}}{r} \frac{m}{\alpha_{j}}+n-1-\sum_{u}\left\{\frac{\beta_{u}}{r}\right\}-\operatorname{degsh}(s)-[R] .
$$

Note that the first term can be simplified as

$$
\frac{1+\sum_{u} \alpha_{u}}{r} \frac{m}{\alpha_{j}}=\left(1+\sum_{u} \alpha_{u}\right) R .
$$

By the fact that $1 \leq \beta_{u} \leq r$

$$
\sum_{u}\left\{\frac{\beta_{u}}{r}\right\}=\sum_{u} \frac{\beta_{u}}{r}-D_{t}
$$

Then

$$
\operatorname{dim}_{\mathbb{C}}\left(\mathscr{M}_{R}\right)=\sum_{u}\left(\alpha_{u} R-\frac{\beta_{u}}{r}\right)+n-1+D_{t}-\operatorname{degsh}(s)+\{R\} .
$$

Plug in the formula for $\operatorname{degsh}(s)$, we can prove the claim.

We have some easy consequences.

Lemma 5.12. (1) $\operatorname{dim}_{\mathbb{C}}\left(\mathscr{M}_{R}\right)$ increases strictly in $R$;

(2) $\operatorname{dim}_{\mathbb{C}}\left(\mathscr{M}_{R+1}\right)=\operatorname{dim}_{\mathbb{C}}\left(\mathscr{M}_{R}\right)+|\mathfrak{a}|$, where $|\mathfrak{a}|=\sum_{u} \alpha_{u}$.

Proof. (1) Suppose that $R_{1}<R_{2}$, by definition

$$
\tau\left(R_{1}, u\right)<\tau\left(R_{2}, u\right)
$$

hence, $\operatorname{dim}_{\mathbb{C}}\left(\mathscr{M}_{R_{1}}\right) \leq \operatorname{dim}_{\mathbb{C}}\left(\mathscr{M}_{R_{2}}\right)$. On the other hand, there exists certain $j$ such that $\tau\left(R_{2}, j\right)$ is an integer, then $\left[\tau\left(R_{1}, j\right)\right]<\left[\tau\left(R_{2}, j\right)\right]$. We conclude that $\operatorname{dim}_{\mathbb{C}}\left(\mathscr{M}_{R_{1}}\right)<\operatorname{dim}_{\mathbb{C}}\left(\mathscr{M}_{R_{2}}\right)$. This proves (1).

(2) follows from the simple observation that $\tau(R+1, u)=\alpha_{u}+\tau(R, u)$.

Set

$$
\mathscr{R}_{k}=\{R \in \mathscr{R} \mid k<R \leq k+1\}, \quad \tilde{\mathscr{R}}_{k}=\left\{(R, \ell) \in \tilde{\mathscr{R}} \mid R \in \mathscr{R}_{k}\right\} .
$$

Lemma 5.13. We have following formulae.

(1) $\left|\tilde{R}_{k}\right|=|\mathfrak{a}|$;

(2) for $R \in \mathscr{R}_{k}$,

$$
k|\mathfrak{a}| \leq \operatorname{dim}\left(\mathscr{M}_{R}\right)-D_{t}<(k+1)|\mathfrak{a}|
$$

(3) If $\Lambda$ is injective,

$$
\operatorname{dim}\left(\mathscr{M}_{R}\right)-D_{t}=r k(R)-1
$$

Proof. Note that

$$
\tilde{\Lambda}: \Lambda^{-1}\left(\mathscr{R}_{k}\right) \longrightarrow \tilde{\mathscr{R}}_{k}
$$

is a bijection. On the other hand, $(j, a) \in \Lambda^{-1}\left(\mathscr{R}_{k}\right)$ if and only if $k \alpha_{j} \leq a \leq(k+1) \alpha_{j}-1$. Therefore, for each fixed $j$ there are $\alpha_{j}$ pairs, i.e. $\left(j, k \alpha_{j}\right), \ldots,\left(j,(k+1) \alpha_{j}-1\right) \in \Lambda^{-1}\left(\mathscr{R}_{k}\right)$. This implies that the size of $\tilde{\mathscr{R}}_{k}$ is $|\mathfrak{a}|$. We proved (1).

Now we prove (2). We first show that

$$
0 \leq \operatorname{dim}_{\mathbb{C}}\left(\mathscr{M}_{R}\right)-D_{t}<|\mathfrak{a}|, \quad \forall R \in \mathscr{R}_{0} .
$$


First of all $\Lambda(j, 0)=\frac{\beta_{j}}{\alpha_{j} r} \in \mathscr{R}_{0}$ for $1 \leq j \leq n$. Suppose

$$
\frac{\beta_{1}}{\alpha_{1} r}=\ldots=\frac{\beta_{m}}{\alpha_{m} r}<\frac{\beta_{m+1}}{\alpha_{m+1} r} \leq \ldots \leq \frac{\beta_{n}}{\alpha_{n} r} .
$$

Set $R_{\text {min }}=\frac{\beta_{1}}{\alpha_{1} r} \in \mathscr{R}_{0}$. Then $r k^{\circ}\left(R_{\text {min }}\right)=1$ and $r k_{\circ}\left(R_{\text {min }}\right)=m$. Hence

$$
\tau\left(R_{\min }, u\right)=0, \quad \text { when } \quad 1 \leq u \leq m \text {; }
$$

and

$$
-1<\tau\left(R_{\min }, u\right)<0, \text { when } \quad m+1 \leq u \leq n .
$$

By the formula (5.10), we have

$$
\begin{aligned}
\operatorname{dim}_{\mathbb{C}}\left(\mathscr{M}_{R_{\text {min }}}\right)-D_{t} & =m-1=r k_{\circ}\left(R_{\text {min }}\right)-1 \\
& \leq n-1<|\mathfrak{a}| .
\end{aligned}
$$

By (2) of Lemma 5.12,

$$
\operatorname{dim}_{\mathbb{C}}\left(\mathscr{M}_{R_{\min }+1}\right)-D_{t}=m-1+|\mathfrak{a}| .
$$

Note that for any $R \in \mathscr{R}_{0}$ we have

$$
R<R_{\min }+1
$$

Therefore for $1 \leq u \leq n$ we have

$$
\tau(R, u)<\tau\left(R_{\min }+1, u\right) .
$$

In particular for $1 \leq u \leq m$ since $\tau\left(R_{\min }+1, u\right)=\alpha_{u}$ we have

$$
[\tau(R, u)] \leq\left[\tau\left(R_{\min }+1, u\right)\right]-1
$$

We conclude that for any $R \in \mathscr{R}_{0}$

$$
\operatorname{dim}_{\mathbb{C}}\left(\mathscr{M}_{R}\right)-D_{t} \leq \operatorname{dim}_{\mathbb{C}}\left(\mathscr{M}_{R_{\min }+1}\right)-D_{t}-m=|\mathfrak{a}|-1 .
$$

For any $R$ in any $\mathscr{R}_{k}$, we may apply (2) of Lemma 5.12 and induction on $[R]$ to complete the proof.

(3) is an easy consequence of (1) of Lemma 5.12 and (2). In fact, $\operatorname{dim}_{\mathbb{C}}\left(\mathscr{M}_{R}\right)-D_{t}$ increases strictly in $R$ and maps $\mathscr{R}_{0}$ to $[0,|\mathfrak{a}|-1] \cap \mathbb{Z}=\{0,1,2, \ldots,|\mathfrak{a}|-1\}$, but both sets have $|\mathfrak{a}|$-elements. Then the map has no other choice. Moreover, under the hypothesis that $\Lambda$ is injective we know that $r k^{\circ}=r k_{\circ}=r k$ (cf. §5.1.5) and $\operatorname{dim}_{\mathbb{C}} \mathscr{M}_{R_{\text {min }}}=0$.

Lemma 5.14. We have $r k_{\circ}(R+1)=r k_{\circ}(R)+|\mathfrak{a}|$.

Proof. In fact $\Lambda\left(j, a+\alpha_{j}\right)=\Lambda(j, a)+1$ implies that the map $\mathscr{R}_{k} \rightarrow \mathscr{R}_{k+1}, R \mapsto R+1$ is bijective. Then for $R \in \mathscr{R}_{k}$ we have

$$
\begin{aligned}
r k_{\circ}(R+1) & =r k_{\circ}(R)+\sharp\left\{R^{\prime}+1 \mid R^{\prime}<R, R^{\prime} \in \mathscr{R}_{k}\right\}+\sharp\left\{R^{\prime} \in \mathscr{R}_{k} \mid R^{\prime}>R\right\} \\
& =r k_{\circ}(R)+\left|\mathscr{R}_{k}\right| \\
& =r k_{\circ}(R)+|\mathfrak{a}| .
\end{aligned}
$$

We next come to the proof of Theorem 5.10. 
Proof of Theorem 5.10. Suppose that $R_{1}<R_{2}$ are two elements in $\mathscr{R}$. We claim that

$$
\operatorname{dim}_{\mathbb{C}}\left(\mathscr{M}_{R_{1}}\right) \leq \operatorname{dim}_{\mathbb{C}}\left(\mathscr{M}_{R_{2}}\right)-\sharp \Lambda^{-1}\left(R_{2}\right) .
$$

In fact, suppose that

$$
\Lambda^{-1}\left(R_{2}\right)=\left\{\left(j_{1}, a_{1}\right), \ldots,\left(j_{m}, a_{m}\right)\right\} .
$$

Then $\left[\tau\left(R_{1}, u\right)\right] \leq\left[\tau\left(R_{2}, u\right)\right]$ and for $u=j_{1}, \ldots, j_{m}$

$$
\left[\tau\left(R_{1}, u\right)\right] \leq\left[\tau\left(R_{2}, u\right)\right]-1
$$

Then the claim (5.12) follows from the definition of $\tau(R, u)$ in the formula (5.9) (see also (3.7)).

Now suppose that

$$
\mathscr{R}_{0}=\left\{R_{\min }=R_{1}<R_{2}<\ldots<R_{|\mathfrak{a}|}\right\} .
$$

We claim that formula (5.8) is true for $R \in \mathscr{R}_{0}$, i.e,

$$
\operatorname{dim}_{\mathbb{C}}\left(\mathscr{M}_{R_{k}}\right)-D_{t}=r k_{\circ}\left(R_{k}\right)-1, \quad \forall R_{k} \in \mathscr{R}_{0} .
$$

This consists of several steps.

Step (i) . Formula (5.8) is true for $R_{\text {min }}=R_{1}$. This is already proved in the proof of Lemma 5.13, see (5.11).

Step(ii). For any $R_{k} \in \mathscr{R}_{0}, 2 \leq k \leq|\mathfrak{a}|$ we have

$$
\operatorname{dim}_{\mathbb{C}}\left(\mathscr{M}_{R_{k}}\right)-D_{t} \geq r k_{\circ}\left(R_{k}\right)-1
$$

Proof. We show for $k=2$. By (5.12) and Step (i),

$$
\begin{aligned}
\operatorname{dim}_{\mathbb{C}}\left(\mathscr{M}_{R_{2}}\right)-D_{t} & \geq \operatorname{dim}_{\mathbb{C}}\left(\mathscr{M}_{R_{\text {min }}}\right)-D_{t}+\left|\Lambda^{-1}\left(R_{2}\right)\right| \\
& =r k_{\circ}\left(R_{\text {min }}\right)-1+\left|\Lambda^{-1}\left(R_{2}\right)\right| \\
& =\left|\Lambda^{-1}\left\{R_{\text {min }}, R_{2}\right\}\right|-1 \\
& =r k_{\circ}\left(R_{2}\right)-1 .
\end{aligned}
$$

The argument can be used inductively to derive the claim for any $3 \leq k \leq|\mathfrak{a}|$.

Step (iii). For any $R_{k} \in \mathscr{R}_{0}, 2 \leq k \leq|\mathfrak{a}|$ we have

$$
\operatorname{dim}_{\mathbb{C}}\left(\mathscr{M}_{R_{k}}\right)-D_{t} \leq r k_{\circ}\left(R_{k}\right)-1
$$

Proof. By (2) of Lemma 5.13, we have already proved that

$$
\operatorname{dim}_{\mathbb{C}}\left(\mathscr{M}_{R_{|\mathfrak{a}|}}\right)-D_{t} \leq|\mathfrak{a}|-1=r k_{\circ}\left(R_{|\mathfrak{a}|}\right)-1 .
$$

This verifies the claim for $k=|\mathfrak{a}|$. By (5.12), we show the claim is true for $k=|\mathfrak{a}|-1$. In fact

$$
\begin{aligned}
\operatorname{dim}_{\mathbb{C}}\left(\mathscr{M}_{R_{|\mathfrak{a}|-1}}\right)-D_{t} & \leq \operatorname{dim}_{\mathbb{C}}\left(\mathscr{M}_{R_{|\mathfrak{a}|}}\right)-D_{t}-\left|\Lambda^{-1}\left(R_{|\mathfrak{a}|}\right)\right| \\
& \leq r k_{\circ}\left(R_{|\mathfrak{a}|}\right)-1-\left|\Lambda^{-1}\left(R_{|\mathfrak{a}|}\right)\right| \\
& =\left|\Lambda^{-1}\left(\mathscr{R}_{0} \backslash\left\{R_{|\mathfrak{a}|}\right\}\right)\right|-1 \\
& =r k_{\circ}\left(R_{|\mathfrak{a}|-1}\right)-1 .
\end{aligned}
$$

The argument can be used inductively to derive the claim for all $2 \leq k \leq|\mathfrak{a}|$.

Combining these three steps, we show that formula (5.8) is true for $R \in \mathscr{R}_{0}$. Then by (2) of Lemma 5.12 and Lemma 5.14 the formula (5.8) is easy to derive for all $R \in \mathscr{R}$. 
5.2. Computation of invariants. In this subsection, we compute the relative invariant

$$
\left\langle\mathbf{R}\left(\tau_{c}\left(\Theta_{(t)}\right) \mid H_{(s)}^{d}\right)\right\rangle=\int_{\mathscr{M}_{R}} \operatorname{ev}_{0}^{*} \Theta_{(t)} \cup \psi^{c} \cup \operatorname{rev}_{\infty}^{*} H_{(s)}^{d}
$$

and prove (3) in Theorem 5.3, i.e.

Theorem 5.15. $\left\langle\mathbf{R}\left(\tau_{c}\left(\Theta_{(t)}\right) \mid H_{(s)}^{d}\right)\right\rangle$ is non-zero.

Let $\mathrm{p}_{o}$ be the origin of $\mathbb{Z}_{r}^{\mathfrak{b}} \ltimes \mathbb{C}^{n}$. Let $\mathscr{M}_{R}^{\circ}$ consist of morphisms that maps $\mathrm{q}_{0}$ to $\mathrm{p}_{o}$. Recall that

$$
\mathscr{M}_{R}=\frac{\operatorname{Hol}_{R}^{0}\left(\mathrm{C}_{o}, \mathbb{Z}_{r}^{\mathfrak{b}} \ltimes \mathbb{C}^{n}\right)}{\mathbb{C}_{o}^{*}} .
$$

Similarly, set $\left(\mathrm{Hol}^{\circ}\right)_{R}^{0}\left(\mathrm{C}_{o}, \mathbb{Z}_{r}^{\mathfrak{b}} \ltimes \mathbb{C}^{n}\right)$ to consists of morphisms that maps $\mathrm{q}_{0}$ to $\mathrm{p}_{o}$. Then

$$
\mathscr{M}_{R}^{\circ}=\frac{\left(\mathrm{Hol}^{\circ}\right)_{R}^{0}\left(\mathrm{C}_{o}, \mathbb{Z}_{r}^{\mathfrak{b}} \ltimes \mathbb{C}^{n}\right)}{\mathbb{C}_{o}^{*}} .
$$

Lemma 5.16. $\left\langle\mathbf{R}\left(\tau_{c}\left(\Theta_{(t)}\right) \mid H_{(s)}^{d}\right)\right\rangle$ can be reduced to be

$$
\mathscr{H}:=\int_{\mathscr{M}_{R}^{\circ}} \psi^{c} \cup \operatorname{rev}_{\infty}^{*} H_{(s)}^{d} .
$$

Proof. The twisted sector of $t$ is

$$
\mathbb{Z}_{r} \ltimes\left(\mathbb{C}^{n}\right)^{\zeta_{r}},
$$

where the action is trivial. The evaluation map is

$$
e v_{0}: \operatorname{Hol}_{R}^{0}\left(C_{0}, \mathbb{Z}_{r}^{\mathfrak{b}} \ltimes \mathbb{C}^{n}\right) \rightarrow\left(\mathbb{C}^{n}\right)^{\zeta_{r}}, \quad \mathrm{u} \mapsto u^{0}\left(\mathrm{q}_{0}\right)
$$

Since $\left(\mathrm{Hol}^{\circ}\right)_{R}^{0}$ is regular, this is a trivial fibration over the neighborhood of $\mathrm{p}_{o}$. Namely,

$$
\left.\operatorname{Hol}_{R}^{0}\left(\mathrm{C}_{0}, \mathbb{Z}_{r}^{\mathfrak{b}} \ltimes \mathbb{C}^{n}\right)\right|_{U} \cong U \times\left(\operatorname{Hol}^{\circ}\right)_{R}^{0}\left(\mathrm{C}_{0}, \mathbb{Z}_{r}^{\mathfrak{b}} \ltimes \mathbb{C}^{n}\right),
$$

where $U$ is a small neighborhood of $\mathrm{p}_{o}$ in $\left(\mathbb{C}^{n}\right)^{\zeta_{r}}$. Therefore

$$
\left.\mathscr{M}_{R}\right|_{U} \cong U \times \mathscr{M}_{R}^{\circ}
$$

Since $\int_{U} \Theta_{(t)}=1$, we have

$$
\int_{\mathscr{M}_{R}} \operatorname{ev}_{0}^{*} \Theta_{(t)} \cup \psi^{c} \cup \operatorname{rev}_{\infty}^{*} H_{(s)}^{d}=\int_{\mathscr{M}_{R}^{\circ}} \psi^{c} \cup \operatorname{rev}_{\infty}^{*} H_{(s)}^{d} .
$$

This proves (5.13).

In this rest of subsection, we compute the integration (5.13). 
5.2.1. Contact orders at $\mathrm{p}_{0} \in \mathrm{P}_{\mathfrak{a}}^{\mathfrak{b}}$ of stable maps. Suppose $r R=\frac{q}{p}$, where $(p, q)=1$ (cf. Lemma 5.6). We introduce contact orders at $p_{0} \in P_{\mathfrak{a}}^{\mathfrak{b}}$ (relative to $q_{0} \in S^{2}$ ) of morphisms in $\mathscr{M}_{R}^{\circ}$. Let

$$
\mathrm{u} \in\left(\mathrm{Hol}^{\circ}\right)_{R}^{0}\left(\mathrm{C}_{o}, \mathbb{Z}_{r}^{\mathfrak{b}} \ltimes \mathbb{C}^{n}\right)
$$

Suppose that $u^{0}: \mathbb{C}_{0} \rightarrow \mathbb{C}^{n}$ is

$$
u^{0}(x)=\left(u_{1}^{0}(x), \ldots, u_{n}^{0}(x)\right)
$$

Roughly speaking, we define the $j$-th contact order of $u$ at $\mathrm{p}_{0}$ to be the vanishing order of $u_{j}^{0}(x)$ at $x=0$.

Let us first consider the case that $u_{j}^{0}(x) \not \equiv 0 . u_{j}^{0}$ defines a morphism

$$
\mathrm{u}_{j}:[\overline{\mathbb{C}}]_{p}^{1} \rightarrow\left[\overline{\mathbb{C}_{j}}\right]_{\alpha_{j}}^{\beta_{j}}
$$

The $j$-th contact order of $\mathrm{u}$ at $\mathrm{p}_{o}$ is defined to be the contact order of $\mathrm{u}_{j}$ at $\mathrm{p}_{0}$. We denote it by $\operatorname{ord}_{j}\left(\mathrm{u}, \mathrm{p}_{0}\right)$. To be explicit,

or

$$
u_{j}[x, y]=\left[\sum_{\ell=0}^{[\tau(R, j)]} c_{\ell} y^{q \alpha_{j}-p\left(\beta_{j}+\ell r\right)} x^{\beta_{j}+\ell r}, y^{q}\right],
$$

$$
u_{j}^{0}(x)=\sum_{\ell=0}^{[\tau(R, j)]} c_{\ell} x^{\beta_{j}+\ell r} .
$$

Definition 5.17. For $\mathrm{u}$ given as above, we define the $\mathrm{j}$-th contact order of $\mathrm{u}$ at $\mathrm{p}_{0}$, denoted by $\operatorname{ord}_{j}\left(\mathrm{u}, \mathrm{p}_{0}\right)$, to be:

- if $\mathrm{u}_{j}(x) \equiv 0$

- if $\mathrm{u}_{j}(x) \not \equiv 0$

$$
\operatorname{ord}_{j}\left(\mathrm{u}, \mathrm{p}_{0}\right):=\frac{\beta_{j}}{r}+[\tau(R, j)]+1
$$

when $c_{k} \neq 0$ and $c_{i}=0$ for $i<k$.

$$
\operatorname{ord}_{j}\left(\mathrm{u}, \mathrm{p}_{0}\right):=\frac{\beta_{j}}{r}+k
$$

Remark 5.18. For each $j$, the $j$-th contact order $c_{j}$ satisfies

$$
c_{\min , j}:=\frac{\beta_{j}}{r} \leq c_{j} \leq \frac{\beta_{j}}{r}+[\tau(R, j)]+1=: c_{\max , j}+1
$$

In particular, when $\operatorname{ord}_{j}(\mathrm{u})=c_{\max , j}+1, \mathrm{u}_{j} \equiv 0$.

Since we assume that $u$ maps $q_{0}$ to $p_{0}$, we find that

$$
\operatorname{ord}_{j}\left(\mathrm{u}, \mathrm{p}_{0}\right) \geq \frac{\beta_{j}}{r}>0
$$

Given $\mathfrak{c}=\left(c_{1}, \ldots, c_{n}\right)$ we define

$$
\mathscr{M}_{R}(\mathfrak{c})=\left\{[\mathrm{u}] \in \mathscr{M}_{R}^{\circ} \mid \operatorname{ord}_{j}\left(\mathrm{u}, \mathrm{p}_{0}\right) \geq c_{j}, 1 \leq j \leq n\right\} .
$$

(Here, we require that $c_{u}-\frac{\beta_{u}}{r}$ is a nonnegative integer). Let

$$
\mathfrak{c}_{\min }=\left(c_{\min , 1}, \ldots, c_{\min , n}\right)
$$

By the definitions, the following lemma is obvious. 
Lemma 5.19. $\mathscr{M}_{R}\left(c_{\text {min }}\right)=\mathscr{M}_{R}^{\circ}$.

On the other hand, suppose that

$$
\Lambda^{-1}(R)=\left\{\left(j_{1}, a_{1}\right), \ldots,\left(j_{m}, a_{m}\right)\right\} .
$$

Let $J=\left\{j_{1}, \ldots, j_{m}\right\}$. Define $\mathfrak{c}_{\max }=\left(c_{1}, \ldots, c_{n}\right)$ to be

$$
c_{u}= \begin{cases}c_{\max , u}+1, & \text { when } u \notin J, \\ c_{\max , u}, & \text { when } u \in J .\end{cases}
$$

Then $\mathscr{M}_{R}\left(\mathfrak{c}_{\max }\right)$ consists of morphisms similar to $\mathrm{u}\left[j_{k}, \beta_{j_{k}}+a_{k} r\right]$. To be precise, the projection of $\mathrm{u}$ to $\left[\overline{\mathbb{C}_{j_{k}}}\right]_{\alpha_{j_{k}}}^{\beta_{j_{k}}}$ is either trivial or $\mathrm{u}\left[j_{k}, \beta_{j_{k}}+a_{k} r\right]$.

5.2.2. Reduce the integration on $\mathscr{M}_{R}\left(\mathfrak{c}_{\min }\right)$ to $\mathscr{M}_{R}\left(\mathfrak{c}_{\max }\right)$. For $\mathfrak{c}=\left(c_{1}, \ldots, c_{n}\right)$, define

$$
\mathfrak{c}_{j}=\left(c_{1}, \ldots, c_{j-1}, c_{j}+1, c_{j+1} \ldots, c_{n}\right) .
$$

When $c_{j} \leq c_{\text {max }, j}, \mathscr{M}_{R}\left(\mathfrak{c}_{j}\right) \subseteq \mathscr{M}_{R}(\mathfrak{c})$ is of codimension 1. As in [24], we interpret $\mathscr{M}_{R}\left(\mathfrak{c}_{j}\right)$ as a zero locus of a transversal section $\sigma$ of a line bundle, denoted by $\mathscr{L}_{\mathfrak{c}_{j}}$, over $\mathscr{M}_{R}(\mathfrak{c})$. We separate the construction into several steps.

Step 1. line bundle $\tilde{\mathscr{L}}_{\mathfrak{c}_{j}}^{0}$ over $\tilde{\mathscr{M}}_{R}^{0}(\mathfrak{c}):=\operatorname{Hol}_{R}^{0}(\mathfrak{c})$.

Let $\mathrm{u} \in \tilde{\mathscr{M}}_{R}^{0}(\mathfrak{c})$. Suppose its restriction on $\mathrm{C}_{0}$ is

$$
\mathrm{u}=\left(u^{0}, u^{1}\right): \mathbb{Z}_{r} \ltimes \mathbb{C}_{o} \rightarrow \mathbb{Z}_{r} \ltimes \mathbb{C}^{n},
$$

where $u^{0}(0)=0$. Let us focus on $u_{j}^{0}: \mathbb{C}_{o} \longrightarrow \mathbb{C}_{j}$. Suppose

$$
c_{j}=\frac{\beta_{j}}{r}+k \text {. }
$$

Then

$$
u_{j}^{0}(s)=a_{j, k} x^{\beta_{j}+k r}+a_{j, k+1} x^{\beta_{j}+(k+1) r}+\ldots
$$

and $a_{j, k} \in\left(\check{\mathbb{C}}_{o}\right)^{\otimes\left(\beta_{j}+k r\right)}$. Define $\tilde{\mathscr{L}}_{\mathfrak{c}_{j}}^{0}$ such that

$$
\left.\tilde{\mathscr{L}}_{\mathfrak{c}_{j}}^{0}\right|_{\mathrm{u}}=\left(\check{C}_{o}\right)^{\otimes\left(\beta_{j}+k r\right)} .
$$

Mean while, $\tilde{\sigma}^{0}(\mathrm{u})=a_{j, k}$ defines a section of this line bundle such that

$$
\left(\tilde{\sigma}^{0}\right)^{-1}(0)=\tilde{\mathscr{M}}_{R}^{0}\left(\mathfrak{c}_{j}\right) .
$$

Obviously, $\tilde{\sigma}^{0}$ is transversal on $\tilde{\mathscr{M}}_{R}^{0}\left(\mathfrak{c}_{j}\right)$ since $a_{j, k}$ can be arbitrary.

Step 2. Line bundle $\mathscr{L}_{\mathfrak{c}_{j}}$ over $\mathscr{M}_{R}(\mathfrak{c})=\mathbb{C}_{o}^{*} \ltimes \tilde{\mathscr{M}}_{R}(\mathfrak{c})$.

$\mathbb{C}_{o}^{*}$-action on $\tilde{\mathscr{M}}_{R}^{0}(\mathfrak{c})$ naturally acts on $\tilde{\mathscr{L}}_{\mathfrak{c}_{j}}^{0}$ and $\tilde{\sigma}^{0}$ is a $\mathbb{C}_{o}^{*}$-equivariant section. This leads to a line bundle

$$
\mathscr{L}_{\mathfrak{c}_{j}}=\mathbb{C}_{o}^{*} \ltimes \tilde{\mathscr{L}}_{\mathfrak{c}_{j}}^{0} \rightarrow \mathbb{C}_{o}^{*} \ltimes \tilde{\mathscr{M}}_{R}(\mathfrak{c})=\mathscr{M}_{R}(\mathfrak{c})
$$

and a transversal section $\sigma$ such that

$$
\sigma^{-1}(0)=\mathscr{M}_{R}\left(\mathfrak{c}_{j}\right)
$$

We conclude that 
Lemma 5.20. $\mathscr{M}_{R}\left(\mathfrak{c}_{j}\right) \subseteq \mathscr{M}_{R}(\mathfrak{c})$ is the Euler class of orbifold line bundle $\mathscr{L}_{\mathfrak{c}_{j}}$. Hence

$$
\int_{\mathscr{M}_{R}\left(\mathfrak{c}_{j}\right)}=\int_{\mathscr{M}_{R}(\mathfrak{c})} c_{1}\left(\mathscr{L}_{\mathfrak{c}_{j}}\right) .
$$

Step 3. $\mathscr{L}_{\mathfrak{c}_{j}}$ vs $\mathscr{L}$.

By definitions, we have

$$
\mathscr{L}_{\mathfrak{c}_{j}}=\left.\tilde{\mathscr{L}}^{\otimes r c_{j}}\right|_{\mathscr{M}_{R}(\mathfrak{c})}=\left.\mathscr{L}^{\otimes c_{j}}\right|_{\mathscr{M}_{R}(\mathfrak{c})}
$$

Therefore,

$$
\int_{\mathscr{M}_{R}(\mathfrak{c})} \psi=\frac{1}{c_{j}} \int_{\mathscr{M}_{R}(\mathfrak{c})} c_{1}\left(\mathscr{L}_{\mathfrak{c}_{j}}\right)=\frac{1}{c_{j}} \int_{\mathscr{M}_{R}\left(\mathfrak{c}_{j}\right)} .
$$

Proposition 5.21. Suppose

$$
\Lambda^{-1}(R)=\left\{\left(j_{1}, a_{1}\right), \ldots,\left(j_{m}, a_{m}\right)\right\}
$$

Let $J=\left\{j_{1}, \ldots, j_{m}\right\}$. The integration $\mathscr{H}$ can be reduced as

$$
\mathscr{H}=\prod_{\ell=1}^{n} \frac{1}{c_{\max , \ell} !} \prod_{\ell \in J} c_{\max , \ell} \int_{\mathscr{M}_{R}\left(\mathfrak{c}_{\max }\right)} \psi^{|J|-1-d} \operatorname{rev}_{\infty}^{*} H^{d} .
$$

Here

$$
c_{\max , \ell} !=\prod_{k=0}^{[\tau(R, \ell)]}\left(c_{\max , \ell}-k\right) .
$$

In fact, when $c_{\max , \ell}$ is an integer, it coincides the standard definition, when $c_{\max , \ell}$ is not an integer,

$$
c_{\max , \ell} !=c_{\max , \ell}\left(c_{\max , \ell}-1\right) \ldots\left(c_{\max , \ell}-\left[c_{\max , \ell}\right]\right) .
$$

Proof. For each $\ell \notin J$, from $\mathfrak{c}_{\min }$ to $\mathfrak{c}_{\max }$ we repeat the reduction $[\tau(R, \ell)]+1$-steps. It exhausts $[\tau(R, \ell)]+1$ 's $\psi$ and contributes $c_{\max } !$.

For $\ell \in J$, from $\mathfrak{c}_{\min }$ to $\mathfrak{c}_{\max }$ we repeat the reduction $[\tau(R, \ell)]$-steps. It exhausts $[\tau(R, \ell)]$ 's $\psi$ and contributes $\left(c_{\max }-1\right)$ !.

The number of $\psi$ left is

$$
\begin{aligned}
& c-\sum_{\ell}([\tau(R, \ell)]+1)+|J| \\
= & \left(\operatorname{dim}_{\mathbb{C}}\left(\mathscr{M}_{R}\right)-D_{t}-d\right)-\sum_{\ell}[\tau(R, \ell)]-n+|J| \\
= & |J|-1-d .
\end{aligned}
$$

5.2.3. Computation of invariants.

Theorem 5.22. The formula for $\mathscr{H}$ is

$$
\mathscr{H}=\frac{1}{r} R^{d} \prod_{\ell=1}^{n} \frac{1}{c_{\max } !}=\frac{1}{r} R^{d} \prod_{\ell=1}^{n} \frac{1}{\left(\frac{\beta_{\ell}}{r}+[\tau(R, \ell)]\right) !} .
$$


This is equivalent to show that

$$
\mathscr{H}^{\prime}:=\prod_{\ell \in J} c_{\max , \ell} \int_{\mathscr{M}_{R}\left(\mathfrak{c}_{\max }\right)} \psi^{|J|-1-d} \operatorname{rev}_{\infty}^{*} H^{d}=\frac{1}{r} R^{d} .
$$

For simplicity, we consider the two cases: $d=0$ and $1 \leq d \leq \sharp \Lambda^{-1}(R)=\sharp J$ separately. Case 1: $d=0$.

For simplicity, assume that $(1, a) \in \Lambda^{-1}(R)$. Set

$$
\mathfrak{c}=\left(c_{\max , 1}, c_{\max , 2}+1, \ldots, c_{\max , n}+1\right) .
$$

Then

$$
\mathscr{H}^{\prime}=c_{\text {max }, 1} \int_{\mathscr{M}_{R}(\mathfrak{c})} 1
$$

Now $\mathscr{M}_{R}(\mathfrak{c})$ consists of only one element: the standard map $\mathrm{u}[1, a]$. We know that

$$
\int_{\mathscr{M}_{R}(\mathfrak{c})} 1=\frac{1}{\beta_{1}+a r}=\frac{1}{r} \frac{1}{c_{\max , 1}} .
$$

Hence, combine the computation together we have $\mathscr{H}^{\prime}=1 / r$.

Case 2: $d \neq 0$.

We now compute

$$
\mathscr{H}^{\prime \prime}=\int_{\mathscr{M}_{R}\left(\mathfrak{c}_{\max }\right)} \psi^{|J|-1-d} \operatorname{rev}_{\infty}^{*} H^{d} .
$$

We apply the localization technique. For simplicity, assume that

$$
\Lambda^{-1}(R)=\left\{\left(1, a_{1}\right) \ldots,\left(m, a_{m}\right)\right\} .
$$

The fixed point are $u\left[k, \beta_{k}+a_{k} r\right], 1 \leq k \leq m$. For each $k$ we have the contributions to the localization formula:

- the action weights on the normal direction corresponding to $p_{j}, 1 \leq j \leq m, j \neq k$ are:

$$
\left(\lambda_{j}-\lambda_{0} \alpha_{j}\right)-\left(\lambda_{k}-\lambda_{0} \alpha_{k}\right) \frac{\beta_{j}+a_{j} r}{\beta_{k}+a_{k} r}=\left(\lambda_{j}-\lambda_{0} \alpha_{j}\right)-\left(\lambda_{k}-\lambda_{0} \alpha_{k}\right) \frac{\alpha_{j}}{\alpha_{k}}=\lambda_{j}-\lambda_{k} \frac{\alpha_{j}}{\alpha_{k}}
$$

- contribution from $\psi$ :

- contribution from $H: \lambda_{k} / \alpha_{k}$.

$$
\frac{r}{\beta_{k}+a_{k} r}\left(\lambda_{k}-\alpha_{k} \lambda_{0}\right)
$$

Then we have

- automorphism group: $\frac{1}{\beta_{k}+a_{k} r}$.

$$
r \mathscr{H}^{\prime \prime}=\sum_{k=1}^{m}\left(\frac{r}{\beta_{k}+a_{k} r}\right)^{m-d} \cdot \frac{\left(\lambda_{k}-\alpha_{k} \lambda_{0}\right)^{m-1-d}\left(\frac{\lambda_{k}}{\alpha_{k}}\right)^{d}}{\prod_{j \neq k}\left(\lambda_{j}-\lambda_{k} \frac{\alpha_{j}}{\alpha_{k}}\right)} .
$$

By simplification, and set $\lambda_{j}^{\prime}=\lambda_{j} / \alpha_{j}$ we have

$$
r \mathscr{H}^{\prime \prime}=\frac{1}{\alpha_{1} \ldots \alpha_{m}} \sum_{k=1}^{m}\left(\frac{r \alpha_{k}}{\beta_{k}+a_{k} r}\right)^{m-d} \cdot \frac{\left(\lambda_{k}^{\prime}-\lambda_{0}\right)^{m-1-d}\left(\lambda_{k}^{\prime}\right)^{d}}{\prod_{j \neq k}\left(\lambda_{j}^{\prime}-\lambda_{k}^{\prime}\right)} .
$$


Note that

By using the fact that

$$
\frac{r \alpha_{k}}{\beta_{k}+a_{k} r}=\frac{1}{R}
$$

$$
\sum_{k=1}^{m} \frac{\left(\lambda_{k}^{\prime}-\lambda_{0}\right)^{m-1-d}\left(\lambda_{k}^{\prime}\right)^{d}}{\prod_{j \neq k}\left(\lambda_{j}^{\prime}-\lambda_{k}^{\prime}\right)}=\sum_{k=1}^{m} \frac{\left(\lambda_{k}^{\prime}\right)^{m-1}}{\prod_{j \neq k}\left(\lambda_{j}^{\prime}-\lambda_{k}^{\prime}\right)}=1,
$$

which is a consequence of

$$
\sum_{k=1}^{m} \frac{\left(\lambda_{k}^{\prime}\right)^{d}}{\prod_{j \neq k}\left(\lambda_{j}^{\prime}-\lambda_{k}^{\prime}\right)}=0, \quad \forall 0 \leq d \leq m-2
$$

and the expansion of Vandermonde determinant, we have

$$
r \mathscr{H}^{\prime \prime}=\frac{1}{\alpha_{1} \ldots \alpha_{m}}\left(\frac{1}{R}\right)^{|J|-d} .
$$

Then

$$
\mathscr{H}^{\prime}=\frac{1}{r} \prod_{\ell \in J} \frac{c_{\max , \ell}}{\alpha_{\ell}}\left(\frac{1}{R}\right)^{|J|-d}=\frac{1}{r} R^{d},
$$

here we use the fact that

$$
\frac{c_{\max , \ell}}{\alpha_{\ell}}=R, \quad \forall \ell \in J
$$

Remark 5.23. This completes the proof of (3) in Theorem 5.3. In fact we show that

$$
\left\langle\mathbf{R}\left(\tau_{c}\left(\Theta_{(t)}\right) \mid H_{(s)}^{d}\right)\right\rangle=\frac{1}{r} R^{d} \prod_{\ell=1}^{n} \frac{1}{\left(\frac{\beta_{\ell}}{r}+[\tau(R, \ell)]\right) !} .
$$

We would like to emphasize that $R$ and $d$ are determined by $c$.

5.3. Special case: $E=\Gamma^{\mu} \ltimes \mathbb{C}^{n}$. Suppose that $t$ in $\mathbf{R}$ is $(\gamma), \gamma \in \Gamma$ with order $r$. Consider a morphism

Then for $u: C_{0} \rightarrow \Gamma^{\mu} \ltimes \mathbb{C}^{n}$,

$$
\mathrm{u} \in \mathscr{M}_{\mathbf{R}}\left(\left[\overline{\mathbb{C}^{n}}\right]_{\mathfrak{a}}^{\mu} \mid P_{\mathfrak{a}}^{\mu}\right)
$$

$$
\pi \circ \mathrm{u}: \mathrm{q}_{0} \rightarrow\langle\gamma\rangle \ltimes\{O\}
$$

Suppose that $\gamma$ acts on $\mathbb{C}^{n}$ as weight $\mathfrak{b}=\left(\beta_{1}, \ldots, \beta_{n}\right)$. We find that $\left.u\right|_{\mathbb{C}_{0}}$ is a composition of the following morphisms:

$$
\mathrm{u}: \mathrm{C}_{0} \stackrel{v}{\rightarrow} \mathbb{Z}_{r}^{\mathfrak{b}} \ltimes \mathbb{C}^{n} \hookrightarrow \Gamma^{\mu} \ltimes \mathbb{C}^{n}
$$

where we identify $\langle\gamma\rangle$ with $\mathbb{Z}_{r}$ via $\gamma \leftrightarrow \zeta_{r}$, and therefore

$$
\mathrm{u}: \mathrm{S}^{2} \stackrel{\overline{\mathrm{v}}}{\rightarrow}\left[\overline{\mathbb{C}^{n}}\right]_{\mathfrak{a}}^{\mathfrak{b}} \hookrightarrow\left[\overline{\mathbb{C}^{n}}\right]_{\mathfrak{a}}^{\mu} .
$$

Repeat the arguments in $\S 5.1 .1$, we get the following facts

$$
\begin{aligned}
\operatorname{Hol}_{\mathbf{R}}\left(S^{2},\left[\overline{\mathbb{C}^{n}}\right]_{\mathfrak{a}}^{\mu}\right) & \cong \operatorname{Hol}_{\mathbf{R}}\left(\mathrm{C}_{0}, \Gamma^{\mu} \ltimes \mathbb{C}^{n}\right), \\
\operatorname{Hol}_{\mathbf{R}}\left(\mathrm{C}_{0}, \Gamma^{\mu} \ltimes \mathbb{C}^{n}\right) & \cong C_{\Gamma}(\gamma) \ltimes \operatorname{Hol}_{\mathbf{R}}^{0}\left(C_{0},\langle\gamma\rangle^{\mathfrak{b}} \ltimes \mathbb{C}^{n}\right) .
\end{aligned}
$$

Next, note that we have a central extension

$$
1 \rightarrow\langle\gamma\rangle \cong \mathbb{Z}_{r} \rightarrow C_{\Gamma}(\gamma) \rightarrow C_{\Gamma}^{\prime}(\gamma) \rightarrow 1
$$


and

$$
1 \rightarrow\langle\gamma\rangle \cong \mathbb{Z}_{r} \rightarrow \mathbb{C}_{o}^{*} \rightarrow \mathbb{C}^{*} \rightarrow 1
$$

The first extension then canonically induces the following extension

$$
1 \rightarrow \mathbb{C}_{o}^{*} \rightarrow \mathbb{C}_{o}^{*} \times_{\langle\gamma\rangle} C_{\Gamma}(\gamma) \rightarrow C_{\Gamma}^{\prime}(\gamma) \rightarrow 1
$$

We conclude that

$$
\mathscr{M}_{\mathrm{R}}\left(\mathrm{S}^{2},\left[\overline{\mathbb{C}^{n}}\right]_{\mathfrak{a}}^{\mu}\right)=\frac{\operatorname{Hol}_{\mathrm{R}}\left(\mathrm{C}_{0}, \mathbb{C}^{n} \rtimes \Gamma^{\mu}\right)}{\operatorname{Aut}\left(\mathrm{S}^{2} /\left\{\mathrm{q}_{0}, \mathrm{q}_{\infty}\right\}\right)} \cong \frac{\operatorname{Hol}_{\mathrm{R}}^{0}\left(\mathrm{C}_{0}, \mathbb{C}^{n} \rtimes \mathbb{Z}_{r}^{\mathfrak{b}}\right)}{\mathbb{C}_{o}^{*} \times\langle\gamma\rangle}
$$

We conclude that

Proposition 5.24. $\mathscr{M}_{\mathbf{R}}\left(\left[\overline{\mathbb{C}^{n}}\right]_{\mathfrak{a}}^{\mu}\right)$ is a fibration over $B H$ with an orbifold fiber $\mathscr{M}_{\mathbf{R}}\left(\left[\overline{\mathbb{C}^{n}}\right]_{\mathfrak{a}}^{\mathfrak{b}}\right)$, where $H=C_{\Gamma}^{\prime}(\gamma)=C_{\Gamma}(\gamma) /\langle\gamma\rangle$.

Proof. In fact, we have

$$
\frac{\operatorname{Hol}_{\mathrm{R}}^{0}\left(\mathrm{C}_{0}, \mathbb{C}^{n} \rtimes \mathbb{Z}_{r}^{\mathfrak{b}}\right)}{\mathbb{C}_{o}^{*} \times_{\langle\gamma\rangle} C_{\Gamma}(\gamma)} \cong \frac{\operatorname{Hol}_{\mathrm{R}}^{0}\left(C_{0}, \mathbb{C}^{n} \rtimes \mathbb{Z}_{r}^{\mathfrak{b}}\right) / \mathbb{C}_{0}^{*}}{C_{\Gamma}^{\prime}(\gamma)} \cong \frac{\mathscr{M}_{\mathrm{R}}\left(\left[\overline{\mathbb{C}^{n}}\right]_{\mathfrak{a}}^{\mathfrak{b}}\right)}{C_{\Gamma}^{\prime}(\gamma)}
$$

\subsection{General cases: $E \rightarrow B$.}

5.4.1. Moduli space. Let $\mathrm{u} \in \operatorname{Hol}_{\mathrm{R}}\left(\mathrm{S}^{2}, \overline{\mathrm{E}}_{\mathfrak{a}}\right)$. Suppose that

$$
\pi \circ \mathrm{u}: \mathrm{q}_{0} \rightarrow\langle\gamma\rangle \ltimes\{x\}
$$

where $\gamma \in \Gamma=G_{x}$. Let $\mathrm{B}(t)$ be the twisted sector containg $\langle\gamma\rangle \ltimes\{x\}$. Let $U:=\Gamma \ltimes U_{x}$ be a local orbifold coordinate chart for $B$. Bundle $E$ locally is

$$
\left.\mathrm{E}\right|_{\mathrm{U}} \cong \Gamma \ltimes\left(U_{x} \times \mathbb{C}^{n}\right) \text {. }
$$

Let $\mu$ be the representation of $\Gamma$ action on $\mathbb{C}^{n}$. Then the morphism $\left.u\right|_{C_{0}}$ is a composition of the following morphisms:

$$
\mathrm{u}: \mathrm{C}_{0} \stackrel{\mathrm{v}}{\rightarrow}\{x\} \times\left(\langle\gamma\rangle^{\mathfrak{b}} \ltimes \mathbb{C}^{n}\right) \rightarrow\{x\} \times\left(\Gamma^{\mu} \ltimes \mathbb{C}^{n}\right)
$$

where $\mathfrak{b}$ is the action weight of $\gamma$ on $\mathbb{C}^{n}$. Hence, we have

$$
\mathrm{u}: \mathrm{S}^{2} \stackrel{\bar{v}}{\rightarrow}\{x\} \times\left[\overline{\mathbb{C}^{n}}\right]_{\mathfrak{a}}^{\mathfrak{b}} \rightarrow\{x\} \times\left[\overline{\mathbb{C}^{n}}\right]_{\mathfrak{a}}^{\mu} \hookrightarrow \overline{\mathrm{E}}_{\mathfrak{a}} .
$$

Apply the similar arguments as before, we have

$$
\mathrm{Hol}_{\mathbf{R}}\left(\mathrm{S}^{2}, \overline{\mathrm{E}}_{\mathfrak{a}}\right) \cong \mathrm{Hol}_{\mathbf{R}}\left(\mathrm{C}_{0}, \mathrm{E}\right) \text {. }
$$

Let $\mathrm{B}(t)=\left(B^{1}(t) \rightrightarrows B^{0}(t)\right)$ be the corresponding twisted sector.

Lemma 5.25. Let $\mathrm{Hol}_{\mathrm{R}}^{0}\left(\mathrm{C}_{0}\right.$, $\left.\mathrm{E}\right)$ be as above. Then

(1) $\operatorname{Hol}_{\mathrm{R}}^{0}\left(\mathrm{C}_{0}, \mathrm{E}\right)$ is a fibration

$$
\phi^{0}: \operatorname{Hol}_{\mathbf{R}}^{0}\left(\mathrm{C}_{0}, \mathrm{E}\right) \rightarrow B^{0}(t)
$$

whose fiber is $\operatorname{Hol}_{\mathbf{R}^{\prime}}^{0}\left(C_{0}, \mathbb{Z}_{r}^{\mathfrak{b}} \ltimes \mathbb{C}^{n}\right)$, here $\mathbf{R}^{\prime}$ is naturally determined by $\mathbf{R}$;

(2) $\mathrm{B}(t)$ acts on $\operatorname{Hol}_{\mathrm{R}}^{0}\left(\mathrm{C}_{0}, \mathrm{E}\right)$ naturally, whose based map is $\phi^{0}$;

(3) $\mathrm{Hol}_{\mathrm{R}}\left(\mathrm{C}_{0}, \mathrm{E}\right)=\mathrm{B}(t) \ltimes \operatorname{Hol}_{\mathrm{R}}^{0}\left(\mathrm{C}_{0}, \mathrm{E}\right)$. 
Proof. Define $\phi^{0}$ to be

$$
\left.\mathrm{u} \mapsto \pi \circ \mathrm{u}\right|_{\mathrm{q}_{0}} \in B^{0}(t)
$$

then for $\langle\gamma\rangle \ltimes\{x\} \in B^{0}(t)$, by Proposition 5.24 we know that its preimage is $\operatorname{Hol}_{\mathrm{R}^{\prime}}^{0}\left(\mathrm{~S}^{2},\left[\overline{\mathbb{C}^{n}}\right]_{\mathfrak{a}}^{b}\right)$. This shows (1).

(2) and (3) are basically followed by the definitions. We skip the proof.

Remark 5.26. Let $\mathrm{B}(t)$ be a twisted sector. $B^{0}(t)$ consists of elements in the format $\langle\gamma\rangle \ltimes\{x\}$. Such an element $\langle\gamma\rangle \ltimes\{x\}$ carries a canonical group of arrows $\langle\gamma\rangle \subseteq B^{1}(t)$. Denote the group by $\operatorname{ker}(\langle\gamma\rangle \ltimes\{x\})$. It forms a trivial $\mathbb{Z}_{r}$-bundle $\operatorname{ker}(t) \rightarrow B^{0}(t)$. $\operatorname{ker}(t) \subseteq B^{1}(t)$ is a canonical kernel of $\mathrm{B}(t)$. Let

$$
\mathrm{B}^{\prime}(t)=\left(\frac{B^{1}(t)}{\operatorname{ker}(t)} \rightrightarrows B^{0}(t)\right)
$$

It is known that $\mathrm{B}(t)$ is a $\mathbb{Z}_{r}$-gerbe over $\mathrm{B}^{\prime}(t)$.

Proposition 5.27. $\mathscr{M}_{\mathrm{R}}\left(\overline{\mathrm{E}}_{\mathfrak{a}} \mid \mathrm{P} \mathrm{E}_{\mathfrak{a}}\right)$ is a fibration over $\mathrm{B}^{\prime}(t)$ with fiber $\mathscr{M}_{\mathrm{R}^{\prime}}\left({\overline{\left[\mathbb{C}^{n}\right]_{\mathfrak{a}}}}_{\mid \mathfrak{a}}^{\mid} \mid P_{\mathfrak{a}}^{\mathfrak{b}}\right)$.

Proof. In fact,

$$
\mathscr{M}_{\mathrm{R}}\left(\overline{\mathrm{E}}_{\mathrm{a}} \mid \mathrm{PE} \mathrm{E}_{\mathfrak{a}}\right)=\frac{\mathrm{Hol}_{\mathrm{R}}\left(\mathrm{S}^{2}, \overline{\mathrm{E}}_{\mathrm{a}}\right)}{\operatorname{Aut}\left(\mathrm{S}^{2} \backslash\left\{\mathrm{q}_{0}, \mathrm{q}_{\infty}\right\}\right)}=\frac{\mathrm{B}(t) \ltimes \operatorname{Hol}_{\mathrm{R}}^{0}\left(\mathrm{C}_{0}, \mathrm{E}\right)}{\operatorname{Aut}\left(\mathrm{S}^{2} \backslash\left\{\mathrm{q}_{0}, \mathrm{q}_{\infty}\right\}\right)}
$$

$\mathrm{B}(t)$ is a $\mathbb{Z}_{r}$-gerbe over $\mathrm{B}^{\prime}(t)$, we may extend it with respect to $\operatorname{Aut}\left(\mathrm{S}^{2} \backslash\left\{\mathrm{q}_{0}, \mathrm{q}_{\infty}\right\}\right) \cong \mathbb{C}^{*}$ to get a $\mathbb{C}_{0}^{*}$-gerbe over $\mathrm{B}^{\prime}(t)$, which we denote it by $\tilde{\mathrm{B}}(t)$. In fact, $\tilde{\mathrm{B}}^{1}(t)$ can be obtained by $\mathrm{B}^{1}(t) \times_{\mathbb{Z}_{r}} \mathbb{C}_{o}^{*}$. Then

$$
\mathscr{M}_{\mathrm{R}}=\frac{\mathrm{B}(t) \ltimes \operatorname{Hol}_{\mathrm{R}}^{0}\left(\mathrm{C}_{0}, \mathrm{E}\right)}{\operatorname{Aut}\left(\mathrm{S}^{2} \backslash\left\{\mathrm{q}_{0}, \mathrm{q}_{\infty}\right\}\right)}=\tilde{\mathrm{B}}(t) \ltimes \operatorname{Hol}_{\mathrm{R}}^{0}\left(\mathrm{C}_{0}, \mathrm{E}\right)=\mathrm{B}^{\prime}(t) \ltimes\left(\mathbb{C}_{0}^{*} \ltimes \operatorname{Hol}_{\mathrm{R}}^{0}\left(\mathrm{C}_{0}, \mathrm{E}\right)\right)
$$

Since $\operatorname{Hol}_{\mathrm{R}}^{0}\left(\mathrm{C}_{0}, \mathrm{E}\right) \rightarrow B^{0}(t)$ is a fibration with fiber $\operatorname{Hol}_{\mathrm{R}^{\prime}}^{0}\left(\mathrm{C}_{0},\left[\overline{\mathbb{C}^{n}}\right]_{\mathfrak{a}}^{\mathfrak{b}}\right)$, we know that

$$
\mathbb{C}_{o}^{*} \ltimes \operatorname{Hol}_{\mathrm{R}}^{0}\left(\mathrm{C}_{0}, \mathrm{E}\right) \rightarrow B^{0}(t)
$$

is a fibration with fiber $\mathscr{M}_{\mathrm{R}^{\prime}}\left(\left[\overline{\mathbb{C}^{n}}\right]_{\mathfrak{a}}^{\mathfrak{b}} \mid P_{\mathfrak{a}}^{\mathfrak{b}}\right)$. With $\mathrm{B}^{\prime}(t)$ action, we know that $\mathscr{M}_{\mathrm{R}}\left(\overline{\mathrm{E}}_{\mathfrak{a}} \mid \mathrm{PE} \mathrm{E}_{\mathfrak{a}}\right)$ is a fibration over $\mathrm{B}^{\prime}(t)$ with fiber $\mathscr{M}_{\mathrm{R}^{\prime}}\left(\left[\overline{\mathbb{C}^{n}}\right]_{\mathfrak{a}}^{\mathfrak{b}} \mid \mathrm{P}_{\mathfrak{a}}^{\mathfrak{b}}\right)$.

5.4.2. Certain relative Gromov-Witten invariants. We consider certain relative Gromov-Witten invariants associated to $\mathscr{M}_{\mathrm{R}}\left(\overline{\mathrm{E}}_{\mathfrak{a}} \mid \mathrm{PE} \mathrm{E}_{\mathfrak{a}}\right)$.

To describe the insertions, we make some preparations.

(1) Basis of $H_{C R}^{*}(\mathrm{~B})$.

Recall that

$$
H_{C R}^{*}(\mathrm{~B})=H^{*}(\mathrm{IB})=\bigoplus_{t \in \mathscr{T}^{\mathrm{B}}} H^{*}(\mathrm{~B}(t)) .
$$

For each $t$ let $\sigma_{(t)}$ be a basis of $H^{*}(\mathrm{~B}(t))$ :

$$
\sigma_{(t)}=\left\{\theta_{(t)}^{1}, \ldots, \theta_{(t)}^{k(t)}\right\}
$$

Set

$$
\sigma_{\star}=\bigsqcup_{t \in \mathscr{T}^{\mathrm{B}}} \sigma_{(t)},
$$

which forms a basis of $H_{C R}^{*}(\mathrm{~B})$. 
Let $\sigma^{\star}$ be the dual basis of $\sigma_{\star}$. Then

$$
\sigma^{\star}=\bigsqcup_{t \in \mathscr{T}^{\mathrm{B}}} \sigma^{(t)},
$$

where $\sigma^{(t)}$ is the dual of $\sigma_{(\bar{t})}$. Suppose that

$$
\sigma^{(t)}=\left\{\theta_{1}^{(t)}, \ldots, \theta_{k(t)}^{(t)}\right\}
$$

where $\theta_{i}^{(t)}$ is the dual of $\theta_{(\bar{t})}^{i}$.

(2) Basis of $H_{C R}^{*}\left(\mathrm{PE}_{\mathfrak{a}}\right)$

Let $s \in \mathscr{T}^{\mathrm{PE}_{\mathrm{a}}}$ and $t=\pi_{\mathscr{T}}(s)$ (cf. Lemma 3.6). Then $\mathrm{PE}_{\mathfrak{a}}(s)$ is a projectivization $\mathrm{PE}_{\mathfrak{a}(s)}^{(s)}$ of a rank $r(s)$-vector bundle $\mathrm{E}^{(s)}$ over $\mathrm{B}(t)$ with respect to certain $S_{\mathfrak{a}(s)}^{1}$ action. Set (cf. (3.8))

$$
\Sigma^{(s)}=\left\{\theta_{j}^{(t)} \cup H_{(s)}^{\ell} \mid \theta_{j}^{(t)} \in \sigma^{(t)}, 0 \leq \ell \leq r(s)-1\right\},
$$

which forms a basis of $H^{*}\left(\mathrm{PE}_{\mathfrak{a}}(s)\right)$. Set $\Sigma_{\star}$ to be the union of $\Sigma^{(s)}$. Similarly, let $\Sigma^{\star}$ be the dual of $\Sigma_{\star}$.

(3) Insertions.

We are ready to describe the insertions for the relative Gromov-Witten invariants:

- insertion for marked point $\mathrm{q}_{0}$ : recall that the evaluation map is

$$
\mathrm{ev}_{0}: \mathscr{M}_{\mathrm{R}}\left(\overline{\mathrm{E}}_{\mathfrak{a}} \mid \mathrm{PE} \mathrm{a}_{\mathrm{a}}\right) \rightarrow \mathrm{E}(t) ;
$$

let $\Theta_{(t)}$ be the Thom form of the bundle $\mathrm{E}(t) \rightarrow \mathrm{B}(t)$, the insertion is taken to be

$$
\mathrm{ev}_{0}^{*}\left(\theta_{(t)}^{i} \wedge \Theta_{(t)}\right) \cup \psi^{c} .
$$

Hence, the insertion is supported at $B$.

- insertion for the relative marked point $\mathrm{q}_{\infty}$ : the evaluation map is

$$
\operatorname{rev}_{\infty}: \mathscr{M}_{\mathrm{R}}\left(\overline{\mathrm{E}}_{\mathfrak{a}} \mid \mathrm{PE} \mathrm{E}_{\mathfrak{a}}\right) \rightarrow \mathrm{PE}_{\mathfrak{a}}(s) ;
$$

it is crucial to note that $\pi_{\mathscr{T}}(s)=\bar{t}$ (cf. Remark 5.7). We take the insertion to be

$$
\operatorname{rev}_{\infty}^{*}\left(\theta_{j}^{(\bar{t})} \cup H_{(s)}^{d}\right) \text {. }
$$

Definition 5.28. The pair of insertions $\left(\tau_{c}\left(\theta_{(t)}^{i} \cup \Theta_{(t)}\right), \theta_{j}^{(\bar{t})} \cup H_{(s)}^{d}\right)$ is called proper if

$$
2 c+2 d+\operatorname{deg}\left(\Theta_{(t)}\right)=\operatorname{dim}\left(\mathscr{M}_{\mathbf{R}}\left(\bar{E}_{\mathfrak{a}} \mid P E_{\mathfrak{a}}\right)\right)-\operatorname{dim}(\mathrm{B}(t))=\operatorname{dim}\left(\mathscr{M}_{\mathrm{R}^{\prime}}\left(\left[\overline{\mathbb{C}}^{n}\right]_{\mathfrak{a}}^{\mathfrak{b}} \mid \mathrm{P}_{\mathfrak{a}}^{\mathfrak{b}}\right)\right) .
$$

The last "=" follows from Proposition 5.27.

(4) Invariant $\left\langle\tau_{c}\left(\theta_{(t)}^{i} \cup \Theta_{(t)}\right) \mid \theta_{j}^{(\bar{t})} \cup H_{(s)}^{d}\right\rangle$ for a pair of proper insertions.

We compute the integration.

$$
\begin{aligned}
& \left\langle\left(\tau_{c}\left(\theta_{(t)}^{i} \cup \Theta_{(t)}\right)\left|\theta_{j}^{(\bar{t})} \cup H_{(s)}^{d}\right\rangle\right.\right. \\
= & \int_{\mathscr{M}_{\mathbf{R}}} \psi^{c} \cup \operatorname{ev}_{0}^{*}\left(\theta_{(t)}^{i}\right) \cup \operatorname{ev}_{0}^{*} \Theta_{(t)} \cup \operatorname{rev}_{\infty}^{*}\left(\theta_{j}^{(\bar{t})}\right) \cup \operatorname{rev}_{\infty}^{*}\left(H_{(s)}^{d}\right)
\end{aligned}
$$




$$
\begin{aligned}
& =\int_{\mathrm{B}^{\prime}(t)} \operatorname{ev}_{0}^{*}\left(\theta_{(t)}^{i}\right) \cup \operatorname{rev}_{\infty}^{*}\left(\theta_{j}^{(\bar{t})}\right) \cdot \int_{\mathscr{M}_{\mathrm{R}^{\prime}}} \psi^{c} \cup \operatorname{ev}_{0}^{*} \Theta_{(t)} \cup \operatorname{rev}_{\infty}^{*} H_{(s)}^{d} \\
& =r \delta_{j}^{i} \cdot \int_{\mathscr{M}_{\mathrm{R}^{\prime}}} \psi^{c} \cup \operatorname{ev}_{0}^{*} \Theta_{(t)} \cup \operatorname{rev}_{\infty}^{*}\left(H_{(s)}^{d}\right) .
\end{aligned}
$$

Here for the first "=" we use Proposition 5.27; for the second "=" we use the definition of properness of the insertions; and $\delta_{j}^{i}$ is the Kronecker number.

We conclude that

Theorem 5.29. Let $\left(\tau_{c}\left(\theta_{(t)}^{i} \cup \Theta_{(t)}\right), \theta_{j}^{(\bar{t})} \cup H_{(s)}^{d}\right)$ be a proper insertion for $\mathscr{M}_{\mathbf{R}}$. The invariant

$$
\left\langle\mathbf{R}\left(\tau_{c}\left(\theta_{(t)}^{i} \cup \Theta_{(t)}\right) \mid \theta_{j}^{(\bar{t})} \cup H_{(s)}^{d}\right)\right\rangle
$$

is non-zero if

- $\theta_{(t)}^{i}$ and $\theta_{j}^{(\bar{t})}$ are dual; and

- $\left\langle\mathbf{R}^{\prime}\left(\tau_{c}\left(\Theta_{(t)}\right) \mid H_{(s)}^{d}\right)\right\rangle$ is the invariant computed in $\$ 5.2$, i.e, $\mathbf{R}^{\prime}$ and d are uniquely determined by $c$.

\section{WEIGHTED-BLOWUP CORRESPONDENCE FOR ORBIFOLD GROMOV-WITTEN INVARIANTS}

In this section we first consider admissible relative data $\mathscr{R}_{\Sigma_{\star}}^{\bullet}(\mathrm{X} \mid \mathrm{Z})$ of a general relative pair $(X \mid Z)$ with relative insertions coming from a choosing basis $\Sigma_{\star}$ of $H_{C R}^{*}(Z)$. We give a partial order to $\mathscr{R}_{\Sigma_{+}}^{\bullet}(X \mid Z)$. Then we consider the pair $\left(\underline{X}_{a} \mid Z\right)$ obtained by blowing up $X$ along a symplectic sub-orbifold groupoid $S$ with weight $\mathfrak{a}$. There is a subset $\mathscr{R}_{\Sigma_{\star}, \mathscr{K}}\left(\underline{X}_{\mathfrak{a}} \mid Z\right)$ of $\mathscr{R}_{\Sigma_{\star}}^{\bullet}\left(\underline{X}_{\mathfrak{a}} \mid Z\right)$ in which all relative datum have absolute insertions coming from $\mathscr{K}$, which is the image of the Chen-Ruan cohomology of $X$ via the natural projection $\kappa: \underline{X}_{\mathfrak{a}} \rightarrow X$ (cf. §3.3 and §6.2).

6.1. Partial order on $\mathscr{R}_{\Sigma_{\star}}(\mathrm{X} \mid \mathrm{Z})$. Consider a relative pair $(\mathrm{X} \mid \mathrm{Z})$ and take a basis $\Sigma_{\star}$ of the Chen-Ruan cohomology $H_{C R}^{*}(Z)$. We assume that elements in $\Sigma_{\star}$ are homogenous with respect to the degree and the decomposition of the inertia sapce IZ. Denote the dual basis by $\Sigma^{\star}$. In this section we give a partial order on the set $\mathscr{R}_{\Sigma_{\star}}^{\bullet}(X \mid Z)$ of admissible relative $\Sigma_{\star}$-data of $(X \mid Z)$.

Given the relative pair $(X \mid Z)$, by blowing up $X$ along $Z$ with trivial weight $\mathfrak{a}=(1)$ we degenerate $(X \mid Z)$ into

$$
(X \mid Z) \stackrel{\text { degenerate }}{\longrightarrow}\left(X^{-} \mid Z^{-}\right) \wedge_{Z}\left(Z^{+}\left|X^{+}\right| Z\right)=(X \mid Z) \wedge_{Z}\left(Z_{\infty}|\bar{N}| Z_{0}\right)
$$

here $\bar{N}$ is the weight $\mathfrak{a}=(1)$ projectification of the normal bundle $N_{Z \mid X}$ of $Z$ in $X$. To distinguish different divisors in $\bar{N}$, we write the original $Z$ in $X$ by $Z_{0} \in \bar{N}$, which is the zero section of $\bar{N}$. We write the infinity section of $\bar{N}$ by $Z_{\infty}$. By gluing $Z_{\infty}$ with the $Z \in X$ we get the original $(X \mid Z)$. Denote the fiber class of $\bar{N} \rightarrow Z$ by $\left[F_{Z}\right]$.

In the next we also consider relative $\left(\Sigma^{\star}, \Sigma_{\star}\right)$-data of $\left(Z_{\infty}|\bar{N}| Z_{0}\right)$. A relative $\left(\Sigma^{\star}, \Sigma_{\star}\right)$-data

$$
\mathbf{R}^{+, \bullet}\left(J_{\infty}|I| J_{0}\right)
$$

consists of

- topological data

$$
\mathbf{R}^{+, \bullet}=\left(g, A, \mathfrak{t}=\left(t_{1}, \ldots, t_{k}\right) \mid \mathfrak{r}_{\infty}, \mathfrak{r}_{0}\right)
$$


with

$$
\mathfrak{r}_{\infty}=\left(\left(s_{\infty}^{1}, u_{\infty}^{1}\right), \ldots,\left(s_{\infty}^{h_{\infty}}, u_{\infty}^{h_{\infty}}\right)\right), \quad \mathfrak{r}_{0}=\left(\left(s_{0}^{1}, u_{0}^{1}\right), \ldots,\left(s_{0}^{h_{0}}, u_{0}^{h_{0}}\right)\right)
$$

- absolute insertions $I=\left(\tau_{d_{1}} \alpha_{1}, \ldots, \tau_{d_{k}} \alpha_{k}\right)$ with $\alpha_{i} \in H^{*}\left(\overline{\mathrm{N}}_{\left(t_{i}\right)}\right)$,

- relative insertions $\mathfrak{i}\left(J_{\infty}\right)=\left(\beta_{\infty}^{1}, \ldots, \beta_{\infty}^{h_{\infty}}\right)$ with $\beta_{\infty}^{i} \in \Sigma^{\star}$ and $\mathfrak{i}\left(J_{0}\right)=\left(\beta_{0}^{1}, \ldots, \beta_{0}^{h_{0}}\right)$ with $\beta_{0}^{i} \in \Sigma_{\star}$.

Then $J_{\infty}=\left(\mathfrak{r}_{\infty}, \mathfrak{i}\left(J_{\infty}\right)\right)$ and $J_{0}=\left(\mathfrak{r}_{0}, \mathfrak{i}\left(J_{0}\right)\right)$.

As in Section 4 , we call a relative $\left(\Sigma^{\star}, \Sigma_{\star}\right)$-data admissible if the total degree of insertions matches the virtual dimension of the corresponding relative moduli space. Denote the set of admissible relative $\left(\Sigma^{\star}, \Sigma_{\star}\right)$-data of $\left(Z_{\infty}|\bar{N}| Z_{0}\right)$ by $\mathscr{R}_{\Sigma^{\star}, \Sigma_{\star}}^{\bullet}\left(Z_{\infty}|\bar{N}| Z_{0}\right)$.

There is a special kind of admissible $\left(\Sigma^{\star}, \Sigma_{\star}\right)$-data of $\left(Z_{\infty}|\bar{N}| Z_{0}\right)$.

Definition 6.1. A connected pre- $\bar{N}$-minimal data in $\mathscr{R}_{\Sigma^{\star}, \Sigma_{\star}}^{\bullet}\left(Z_{\infty}|\bar{N}| Z_{0}\right)$ is a connected admissible data $\mathbf{R}^{+}\left(J_{\infty}|\varnothing| J_{0}\right)$ whose topological data is of the form

$$
\mathbf{R}^{+}=\left(g=0, A=u \cdot\left[F_{Z}\right], \mathfrak{t}=\varnothing \mid \mathfrak{r}_{\infty}=\left(s_{\infty}, u\right), \mathfrak{r}_{0}=\left(\bar{s}_{\infty}, u\right)\right) .
$$

We call a connected pre- $\overline{\mathrm{N}}$-minimal data an $\overline{\mathrm{N}}$-minimal data if $\mathfrak{i}\left(\breve{J}_{\infty}\right)=\mathfrak{i}\left(J_{0}\right)$, hence $\breve{J}_{\infty}=J_{0}$.

A disconnected pre- $\bar{N}$-minimal $\left(\bar{N}\right.$-minimal resp.) data of $\left(Z_{\infty}|\bar{N}| Z_{0}\right)$ is a disjoint union of finite connected pre- $\overline{\mathrm{N}}$-minimal ( $\overline{\mathrm{N}}$-minimal resp.) datum.

The computation in $\S 5$ and Theorem 5.29 implies that

Proposition 6.2. Let $\mathbf{R}^{+}\left(J_{\infty}|\varnothing| J_{0}\right)$ be a connected pre- $\overline{\mathrm{N}}$-minimal data. Then the invariant

$$
\left\langle\mathbf{R}^{+}\left(J_{\infty}|\varnothing| J_{0}\right)\right\rangle^{Z_{\infty}|\bar{N}| Z_{0}} \neq 0
$$

if and only if $\mathbf{R}^{+}\left(J_{\infty}|\varnothing| J_{0}\right)$ is $\overline{\mathrm{N}}$-minimal, i.e. $J_{\infty}=\breve{J}_{0}$.

Remark 6.3. For completeness we also call empty data

$$
\left(g=0, A=0, \mathfrak{t}=\varnothing \mid \mathfrak{r}_{\infty}=\varnothing, \mathfrak{r}_{0}=\varnothing\right) .
$$

$\overline{\mathrm{N}}$-minimal, and set its invariant to be 1. Note that when $A=0, \mathfrak{r}_{\infty}$ and $\mathfrak{r}_{0}$ are both empty.

6.1.1. Definition of partial order. Now we define the partial order on $\mathscr{R}_{\Sigma_{\star}}(\mathrm{X} \mid \mathrm{Z})$.

Definition 6.4. Take two admissible $\Sigma_{\star}$-datum $\mathbf{R}_{i}^{\bullet}\left(I_{i} \mid J_{i}\right) \in \mathscr{R}_{\Sigma_{\star}}^{\bullet}(\mathrm{X} \mid \mathrm{Z}), i=1,2$. We say that

$$
\mathbf{R}_{1}^{\bullet}\left(I_{1} \mid J_{1}\right) \prec \mathbf{R}_{2}^{\bullet}\left(I_{2} \mid J_{2}\right)
$$

if when we degenerate $(\mathrm{X} \mid \mathrm{Z})$ as $(6.1)$, there is an admissible $\left(\Sigma^{\star}, \Sigma_{\star}\right)$-data $\mathbf{R}^{+, \bullet}\left(J_{\infty}|I| J_{0}\right) \in$ $\mathscr{R}_{\Sigma^{\star}, \Sigma_{\star}}^{\bullet}\left(\mathrm{Z}_{\infty}|\overline{\mathrm{N}}| \mathrm{Z}_{0}\right)$ such that

(P1) the pair

$$
\left(\mathbf{R}_{1}^{\bullet}\left(I_{1} \mid J_{1}\right), \mathbf{R}^{+\bullet}\left(J_{\infty}|I| J_{0}\right)\right)
$$

is a matched pair, i.e. $J_{1}=\breve{J}_{\infty}$ and

$$
\mathbf{R}_{2}^{\bullet}\left(I_{2} \mid J_{2}\right)=\mathbf{R}_{1}^{\bullet}\left(I_{1} \mid J_{1}\right) * \mathbf{R}^{+, \bullet}\left(J_{\infty}|I| J_{0}\right)
$$

hence $J_{2}=J_{0}$, and moreover

(P2) when $\mathbf{R}^{+, \bullet}\left(J_{\infty}|I| J_{0}\right)$ is pre- $\overline{\mathrm{N}}$-minimal, it must be $\overline{\mathrm{N}}$-minimal. Then $J_{2}=J_{0}=\breve{J}_{\infty}=J_{1}$ and $\mathbf{R}_{2}^{\bullet}\left(I_{2} \mid J_{2}\right)=\mathbf{R}_{1}^{\bullet}\left(I_{1} \mid J_{1}\right)$. (Here we allow empty $\overline{\mathrm{N}}$-minimal data). 
The main theorem in this subsection is the following result.

Theorem 6.5. $\left(\mathscr{R}_{\Sigma_{\star}}^{\bullet}(\mathrm{X} \mid \mathrm{Z}), \prec\right)$ is a partial ordered set.

6.1.2. Proof of Theorem 6.5. Before giving the proof of Theorem 6.5 we give some lemmas.

Lemma 6.6. Let $\mathbf{R}^{\bullet}(I \mid J) \in \mathscr{R}_{\Sigma_{\star}}^{\bullet}(X \mid Z)$. Then there exists a unique $\overline{\mathrm{N}}$-minimal data $\mathbf{R}^{+, \bullet}(\breve{J}|\varnothing| J) \in$ $\mathscr{R}_{\Sigma^{\star}, \Sigma_{\star}}^{\bullet}\left(Z_{\infty}|\overline{\mathrm{N}}| \mathrm{Z}_{0}\right)$ such that

$$
\mathbf{R}^{\bullet}(I \mid J) * \mathbf{R}^{+, \bullet}(\breve{J}|\varnothing| J)=\mathbf{R}^{\bullet}(I \mid J)
$$

Conversely, if there is another data $\mathbf{R}^{+, \bullet}\left(J_{\infty}\left|I^{+}\right| J_{0}\right) \in \mathscr{R}_{\Sigma^{\star}, \Sigma_{\star}}^{\bullet}\left(\mathrm{Z}_{\infty}|\overline{\mathrm{N}}| \mathrm{Z}_{0}\right)$ satisfying (6.3) and the associated invariant is nonzero, then it must be the $\overline{\mathrm{N}}$-minimal data $\mathbf{R}^{+, \bullet}(\breve{J}|\varnothing| J)$ in (6.3). Proof. Suppose that the relative data of $\mathbf{R}^{\bullet}(I \mid J)$ is

$$
\mathbf{R}^{\bullet}=\left(g, A, \mathfrak{t}=\left(t_{1}, \ldots, t_{m}\right) \mid \mathfrak{r}=\left(\left(s_{1}, u_{1}\right), \ldots,\left(s_{h}, u_{h}\right)\right)\right),
$$

and the relative insertions are $\mathrm{i}(J)=\left(\beta_{1}, \ldots, \beta_{h}\right)$ with $\beta_{k} \in \Sigma_{\star}$.

The $\overline{\mathrm{N}}$-minimal data $\mathbf{R}^{+, \bullet}(\breve{J}|\varnothing| J)$ is constructed as follow. It consists of of $h=\sharp J$ connected components with $i$-th component $\mathbf{R}_{i}^{+}\left(\breve{J}_{i}|\varnothing| J_{i}\right)$ being of the following form:

- topological data

$$
\mathbf{R}_{i}^{+}=\left(g=0, A_{i}^{+}=u_{i} \cdot\left[F_{Z}\right], \mathfrak{t}=\varnothing \mid \mathfrak{r}_{0}=\left(s_{i}, u_{i}\right), \mathfrak{r}_{\infty}=\left(\left(\bar{s}_{i}, u_{i}\right)\right)\right),
$$

- the relative insertions are $\check{\beta}_{i}$ for $Z_{\infty}$ and $\beta_{i}$ for $Z_{0}$.

When $\mathfrak{r}=\varnothing$, all $\mathbf{R}_{i}^{+}\left(\check{J}_{i}|\varnothing| J_{i}\right)$ are the empty $\overline{\mathrm{N}}$-minimal data.

Obviously, $\mathbf{R}_{i}^{+}\left(\breve{J}_{i}|\varnothing| J_{i}\right)$ is $\overline{\mathrm{N}}$-minimal. Hence

$$
\mathbf{R}^{+, \bullet}(\breve{J}|\varnothing| J)=\bigsqcup_{i=1}^{h} \mathbf{R}_{i}^{+}\left(\breve{J}_{i}|\varnothing| J_{i}\right)
$$

is $\overline{\mathrm{N}}$-minimal. Moreover, one can directly see that

$$
\mathbf{R}^{\bullet}(I \mid J)=\mathbf{R}^{\bullet}(I \mid J) * \mathbf{R}^{+, \bullet}(\breve{J}|\varnothing| J) .
$$

Conversely, suppose there is another data $\mathbf{R}^{+, \bullet}\left(J_{\infty}\left|I^{+}\right| J_{0}\right) \in \mathscr{R}_{\Sigma^{\star}, \Sigma_{\star}}^{\bullet}\left(Z_{\infty}|\bar{N}| Z_{0}\right)$ such that

$$
\mathbf{R}^{\bullet}(I \mid J) * \mathbf{R}^{+, \bullet}\left(J_{\infty}\left|I^{+}\right| J_{0}\right)=\mathbf{R}^{\bullet}(I \mid J) .
$$

We first consider the case that $\mathbf{R}^{+, \bullet}\left(J_{\infty}\left|I^{+}\right| J_{0}\right)$ is not empty. Then

- the homology class of $\mathbf{R}^{+, \bullet}\left(J_{\infty}\left|I^{+}\right| J_{0}\right)$ must be a fiber class $A^{+}=\sum_{i} u_{i} \cdot\left[F_{Z}\right]$;

- the genus of each connected component is zero;

- $J_{0}=J, \breve{J}_{\infty}=J, I^{+}=\varnothing$.

Since the genus of each component is zero, the homology class is a fiber class, every connected component of $\mathbf{R}^{+, \bullet}\left(J_{\infty}\left|I^{+}\right| J_{0}\right)$ would have exactly one relative marking mapped to each one of $Z_{0}, Z_{\infty}$. So $\mathbf{R}^{+, \bullet}\left(J_{\infty}\left|I^{+}\right| J_{0}\right)$ has exactly $\sharp J$ components and every component is pre- $\overline{\mathrm{N}}$ minimal. Then the invariant of $\mathbf{R}^{+, \bullet}\left(J_{\infty}\left|I^{+}\right| J_{0}\right)$ is the product of the invariant of each component. Therefore by $J=J_{0}=\breve{J}_{\infty}$, Proposition 6.2 and the assumption $\left\langle\mathbf{R}^{+, \bullet}\left(J_{\infty}\left|I^{+}\right| J_{0}\right)\right\rangle \neq 0$, we see that each component of $\mathbf{R}^{+, \bullet}\left(J_{\infty}\left|I^{+}\right| J_{0}\right)$ is $\overline{\mathrm{N}}$-minimal. Hence $\mathbf{R}^{+, \bullet}\left(J_{\infty}\left|I^{+}\right| J_{0}\right)$ is $\overline{\mathrm{N}}$-minimal. One can then easily see that $\mathbf{R}^{+, \bullet}\left(J_{\infty}\left|I^{+}\right| J_{0}\right)=\mathbf{R}^{+, \bullet}(\breve{J}|\varnothing| J)$. This also proves the uniqueness of $\mathbf{R}^{+, \bullet}(\breve{J}|\varnothing| J)$.

When $J=\varnothing$, we take $\mathbf{R}^{+, \bullet}(\breve{J}|\varnothing| J)$ to be the empty data in Remark 6.3. 
We can also degenerate $\left(Z_{\infty}|\bar{N}| Z_{0}\right)$ by blowing up $\bar{N}$ along $Z_{0}$ with trivial weight. This procedure degenerates $\left(Z_{\infty}|\bar{N}| Z_{0}\right)$ into two copies of itself

$$
\left(Z_{\infty}|\bar{N}| Z_{0}\right) \stackrel{\text { degenerate }}{\longrightarrow}\left(Z_{\infty}|\bar{N}| Z_{0}\right) \wedge_{Z}\left(Z_{\infty}|\bar{N}| Z_{0}\right)
$$

By gluing $Z_{0}$ in the first copy and $Z_{\infty}$ in the second copy we get the original $\left(Z_{\infty}|\bar{N}| Z_{0}\right)$.

Lemma 6.7. Let $\mathbf{R}^{\bullet}\left(J_{\infty}|\varnothing| J_{0}\right)$ be a pre- $\overline{\mathrm{N}}$-minimal data. Suppose when we degenerate $\left(\mathrm{Z}_{\infty}|\overline{\mathrm{N}}| \mathrm{Z}_{0}\right)$ as (6.4) there holds

$$
\mathbf{R}^{\bullet}\left(J_{\infty}|\varnothing| J_{0}\right)=\mathbf{R}^{-, \bullet}\left(J_{\infty}|\varnothing| J_{0}^{-}\right) * \mathbf{R}^{+, \bullet}\left(J_{\infty}^{+}|\varnothing| J_{0}\right)
$$

for some $\left(\Sigma^{\star}, \Sigma_{\star}\right)$-datum of $\left(\mathrm{Z}_{\infty}|\overline{\mathrm{N}}| \mathrm{Z}_{0}\right)$. Then both datum $\mathbf{R}^{-, \bullet}\left(J_{\infty}^{-}|\varnothing| J_{0}^{-}\right)$and $\mathbf{R}^{+, \bullet}\left(J_{\infty}^{+}|\varnothing| J_{0}^{+}\right)$ are pre- $\overline{\mathrm{N}}$-minimal.

Suppose further more that $\mathbf{R}^{\bullet}\left(J_{\infty}|\varnothing| J_{0}\right)$ is $\overline{\mathrm{N}}$-minimal and, one of $\mathbf{R}^{-, \bullet}\left(J_{\infty}^{-}|\varnothing| J_{0}^{-}\right)$and $\mathbf{R}^{+, \bullet}\left(J_{\infty}^{+}|\varnothing| J_{0}^{+}\right)$ is also $\overline{\mathrm{N}}$-minimal. Then

$$
\mathbf{R}^{\bullet}\left(J_{\infty}|\varnothing| J_{0}\right)=\mathbf{R}^{-, \bullet}\left(J_{\infty}|\varnothing| J_{0}^{-}\right)=\mathbf{R}^{+, \bullet}\left(J_{\infty}^{+}|\varnothing| J_{0}\right)
$$

and are all $\overline{\mathrm{N}}$-minimal.

Proof. We only have to consider the connected and nonempty case. By the assumption we write the topological datum of $\mathbf{R}\left(J_{\infty}|\varnothing| J_{0}\right), \mathbf{R}^{+}\left(J_{\infty}|\varnothing| J_{0}^{+}\right)$, and $\mathbf{R}^{-}\left(J_{\infty}^{-}|\varnothing| J_{0}\right)$ as

$$
\begin{aligned}
& \mathbf{R}=\left(g=0, A=u \cdot\left[F_{Z}\right], \mathfrak{t}=\varnothing \mid \mathfrak{r}_{\infty}=\left(s_{\infty}, u\right), \mathfrak{r}_{0}=\left(\bar{s}_{\infty}, u\right)\right), \\
& \mathbf{R}^{+}=\left(g^{+}, A^{+}, \mathfrak{t}^{+}=\varnothing \mid \mathfrak{r}_{\infty}=\left(s_{\infty}, u\right), \mathfrak{r}_{0}^{+}=\left(s_{0}^{+}, u_{0}^{+}\right)\right), \\
& \mathbf{R}^{-}=\left(g^{-}, A^{-}, \mathfrak{t}^{+}=\varnothing \mid \mathfrak{r}_{\infty}^{-}=\left(s_{\infty}^{-}, u_{\infty}^{-}\right), \mathfrak{r}_{0}=\left(\bar{s}_{\infty}, u\right)\right) .
\end{aligned}
$$

Then we have

$$
\begin{aligned}
& \text { - } A=A^{+}=A^{-}, g^{+}=g^{-}=g=0 ; \\
& \text { - } s_{\infty}=\bar{s}_{0}^{+}, s_{0}^{+}=\bar{s}_{\infty}^{-}, s_{\infty}^{-}=s_{\infty} ; \\
& \text { - } u=u_{0}^{+}=u_{\infty}^{-} .
\end{aligned}
$$

Hence $\mathbf{R}^{-, \bullet}\left(J_{\infty}|\varnothing| J_{0}^{-}\right)$and $\mathbf{R}^{+, \bullet}\left(J_{\infty}^{+}|\varnothing| J_{0}\right)$ both are pre- $\overline{\mathrm{N}}$-minimal. This proves the first part.

We next prove the second assertion. Without loss of generality we assume that $\mathbf{R}^{\bullet}\left(J_{\infty}|\varnothing| J_{0}\right)$, and $\mathbf{R}^{-, \bullet}\left(J_{\infty}|\varnothing| J_{0}^{-}\right)$are $\overline{\mathrm{N}}$-minimal. Therefore

$$
J_{\infty}=\breve{J}_{0}, \quad J_{\infty}=\check{J}_{0}^{-} .
$$

Combining with $J_{0}^{-}=\breve{J}_{\infty}^{+}$we get

$$
J_{\infty}=J_{\infty}^{+}=\breve{J}_{0}=\breve{J}_{0}^{-} .
$$

So $\mathbf{R}^{-}\left(J_{\infty}|\varnothing| J_{0}^{-}\right)=\mathbf{R}^{+}\left(J_{\infty}^{+}|\varnothing| J_{0}\right)=\mathbf{R}\left(J_{\infty}|\varnothing| J_{0}\right)$ and are all $\overline{\mathrm{N}}$-minimal.

This lemma says that if we consider the partial order, similar as the one given in Definition 6.4, on $\mathscr{R}_{\Sigma^{\star}, \Sigma_{\star}}\left(Z_{\infty}|\bar{N}| Z_{0}\right), \bar{N}$-minimal datum are minimal elements.

Now we give the proof of Theorem 6.5.

Proof of Theorem 6.5. The proof consists of several steps.

Step 1: Reflexivity. This is the first part of Lemma 6.6. Hence " $\prec$ " is reflexive. 
Step 2: Transitivity. Let $\mathbf{R}_{i}=\mathbf{R}_{i}\left(I_{i} \mid J_{i}\right) \in \mathscr{R}_{\Sigma_{\star}}^{\bullet}(\mathrm{X} \mid \mathrm{Z}), i=1,2,3$ be three admissible relative datum of (X|Z), $\mathbf{R}_{2} \prec \mathbf{R}_{1}, \mathbf{R}_{3} \prec \mathbf{R}_{2}$, and

$$
\mathbf{R}_{1}=\mathbf{R}_{2} * \mathbf{R}_{a}^{+, \bullet} \text {, and } \mathbf{R}_{2}=\mathbf{R}_{3} * \mathbf{R}_{b}^{+, \bullet}
$$

with

$$
\mathbf{R}_{a}^{+, \bullet}=\mathbf{R}_{a}^{+, \bullet}\left(J_{a}^{+}\left|I_{a}\right| J_{1}\right), \mathbf{R}_{b}^{+, \bullet}=\mathbf{R}_{b}^{+, \bullet}\left(J_{b}^{+}\left|I_{b}\right| J_{2}\right) \in \mathscr{R}_{\Sigma^{*}, \Sigma_{\star}}^{\bullet}\left(Z_{\infty}|\bar{N}| Z\right)
$$

Here $I_{a}, I_{b}$ are proper extensions of parts of $I_{1}$ and $I_{2}$, and $\mathfrak{i}\left(J_{a}^{+}\right), \mathfrak{i}\left(J_{b}^{+}\right) \in \Sigma^{\star}$. Then since $\left(\mathbf{R}_{2}, \mathbf{R}_{a}^{+, \bullet}\right)$ and $\left(\mathbf{R}_{3}, \mathbf{R}_{b}^{+, \bullet}\right)$ are matched pairs we have

$$
J_{2}=\check{J}_{a}^{+}, \quad J_{3}=\check{J}_{b}^{+} \text {. }
$$

Hence $\mathbf{R}_{b}^{+, \bullet} * \mathbf{R}_{a}^{+, \bullet}$ is an admissible relative $\left(\Sigma^{\star}, \Sigma_{\star}\right)$-data of $\left.\left(Z_{\infty}|\bar{N}| Z_{0}\right)\right)$ and

$$
\mathbf{R}_{3} *\left(\mathbf{R}_{b}^{+, \bullet} * \mathbf{R}_{a}^{+, \bullet}\right)=\mathbf{R}_{1} .
$$

Now if $\mathbf{R}_{b}^{+, \bullet} * \mathbf{R}_{a}^{+, \bullet}$ is pre- $\bar{N}$-minimal, then Lemma 6.7 implies that $\mathbf{R}_{b}^{+, \bullet}, \mathbf{R}_{a}^{+, \bullet}$ are both pre- $\overline{\mathrm{N}}$ minimal. Then by (P2) in Definition 6.4, both $\mathbf{R}_{b}^{+, \bullet}, \mathbf{R}_{a}^{+, \bullet}$ are $\overline{\mathrm{N}}$-minimal. Therefore by Lemma 6.7

$$
\mathbf{R}_{b}^{+, \bullet} * \mathbf{R}_{a}^{+, \bullet}=\mathbf{R}_{b}^{+, \bullet}=\mathbf{R}_{a}^{+, \bullet}
$$

is $\overline{\mathrm{N}}$-minimal. Consequently $\mathbf{R}_{3} \prec \mathbf{R}_{1}$.

Step 3: Antisymmetry. Suppose now we have two admissible relative datum $\mathbf{R}_{1}^{\bullet}\left(I_{1} \mid J_{1}\right)$ and $\mathbf{R}_{2}^{\bullet}\left(I_{2} \mid J_{2}\right)$ of $(\mathrm{X} \mid \mathrm{Z})$. Suppose also that

$$
\begin{aligned}
& \mathbf{R}_{1}^{\bullet}\left(I_{1} \mid J_{1}\right) \prec \mathbf{R}_{2}^{\bullet}\left(I_{2} \mid J_{2}\right), \\
& \mathbf{R}_{2}^{\bullet}\left(I_{1} \mid J_{1}\right) \prec \mathbf{R}_{1}^{\bullet}\left(I_{2} \mid J_{2}\right) .
\end{aligned}
$$

Then as above we have

$$
\mathbf{R}_{1}^{\bullet}=\mathbf{R}_{2}^{\bullet} * \mathbf{R}_{a}^{+, \bullet}, \text { and } \mathbf{R}_{2}^{\bullet}=\mathbf{R}_{1}^{\bullet} * \mathbf{R}_{b}^{+, \bullet}
$$

for some

$$
\mathbf{R}_{a}^{+, \bullet}=\mathbf{R}_{a}^{+, \bullet}\left(J_{a}^{+}\left|I_{a}\right| J_{1}\right), \mathbf{R}_{b}^{+, \bullet}=\mathbf{R}_{b}^{+, \bullet}\left(J_{b}^{+}\left|I_{b}\right| J_{2}\right) \in \mathscr{R}_{\Sigma^{\star}, \Sigma_{\star}}^{\bullet}\left(Z_{\infty}|\bar{N}| Z\right) .
$$

When one of homology classes of $\mathbf{R}_{a}^{+, \bullet}$ and $\mathbf{R}_{b}^{+, \bullet}$ is zero, the corresponding $\left(\Sigma^{\star}, \Sigma_{\star}\right)$-data of $\left(Z_{\infty}|\bar{N}| Z\right)$ is empty, then we have $R_{1}^{\bullet}=R_{2}^{\bullet}$. Therefore in the following we assume that both the homology classes of $\mathbf{R}_{a}^{+, \bullet}$ and $\mathbf{R}_{b}^{+, \bullet}$ are nonzero. Hence $J_{1} \neq \varnothing$. Then we get

$$
\mathbf{R}_{1}^{\bullet}=\mathbf{R}_{1}^{\bullet} *\left(\mathbf{R}_{b}^{+, \bullet} * \mathbf{R}_{a}^{+, \bullet}\right) .
$$

Since $J_{1} \neq \varnothing$, by the second part of Lemma $6.6, \mathbf{R}_{b}^{+, \bullet} * \mathbf{R}_{a}^{+, \bullet}$ is the $\bar{N}$-minimal datum determined by $\mathbf{R}_{1}^{\bullet}$ by the construction in the proof of Lemma 6.6. Therefore by the first part of Lemma 6.7 both $\mathbf{R}_{b}^{+, \bullet}$ and $\mathbf{R}_{a}^{+, \bullet}$ are pre- $\bar{N}$-minimal. Then by (P2) in Definition 6.4 both $\mathbf{R}_{b}^{+, \bullet}$ and $\mathbf{R}_{a}^{+, \bullet}$ must be $\overline{\mathrm{N}}$-minimal. So the second part of Lemma 6.7 implies that

$$
\mathbf{R}_{a}^{+, \bullet}=\mathbf{R}_{b}^{+, \bullet}=\mathbf{R}_{b}^{+, \bullet} * \mathbf{R}_{a}^{+, \bullet}
$$

So we have

$$
\mathbf{R}_{2}^{\bullet}=\mathbf{R}_{1}^{\bullet} * \mathbf{R}_{b}^{+, \bullet}=\mathbf{R}_{1}^{\bullet} *\left(\mathbf{R}_{b}^{+, \bullet} * \mathbf{R}_{a}^{+, \bullet}\right)=\mathbf{R}_{1}^{\bullet} .
$$

Hence " $\prec$ " is also anti-symmetry.

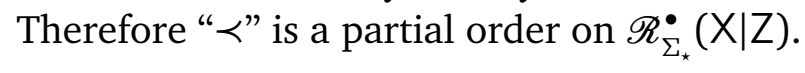

As a direct consequence of the finiteness of $\mathscr{D}_{\mathbf{R}^{\bullet}(I \mid J)}\left(I^{+}, I^{-}\right)$with $I=I^{+} \cup I^{-}$we have 
Lemma 6.8. Given an admissible data $\mathbf{R}^{\bullet}(I \mid J) \in \mathscr{R}_{\Sigma_{x}}(\mathrm{X} \mid \mathrm{Z})$, there are only finite admissible datum $\mathbf{R}^{\prime \bullet}\left(I^{\prime} \mid J^{\prime}\right) \in \mathscr{R}_{\Sigma_{*}}(\mathrm{X} \mid \mathrm{Z})$ such that

$$
\mathbf{R}^{\bullet \bullet}\left(I^{\prime} \mid J^{\prime}\right) \prec \mathbf{R}^{\bullet}(I \mid J) .
$$

6.2. Correspondence. Suppose $S$ is a compact symplectic sub-orbifold groupoid of $X$. We blow up $X$ along $S$ with weight $\mathfrak{a}$ to degenerate $(X, S)$ into (cf. Section 3.3 and Section 4.4)

$$
(X, S) \stackrel{\text { degenerate }}{\longrightarrow}\left(X^{-} \mid Z^{-}\right) \wedge_{Z}\left(X^{+} \mid Z^{+}, S\right)=\left(\underline{X}_{a} \mid Z\right) \wedge_{Z}\left(\bar{N}_{a} \mid Z, S\right)
$$

where $\bar{N}_{\mathfrak{a}}$ and $Z=P N_{\mathfrak{a}}$ are the weight-a projectification and weight-a projectivization of the normal bundle of $\mathrm{S}$ respectively. Then we have a canonical morphism $\kappa: \underline{X}_{\mathfrak{a}} \rightarrow \mathrm{X}$ (cf. §3.3). It induces a morphism on inertia spaces

$$
\mid \kappa=\coprod_{(h) \in \mathscr{T} \underline{\underline{x}}_{a}} \kappa_{(h)}: \coprod_{(h) \in \mathscr{T} \underline{\underline{x}}_{a}} \underline{X}_{\mathfrak{a}}(h) \rightarrow \coprod_{(h) \in \mathscr{T} \underline{\underline{x}}_{a}} X\left(\kappa_{\mathscr{T}}(h)\right)
$$

and homomorphism

$$
\mid \kappa^{*}=\coprod_{(h) \in \mathscr{T} \underline{\underline{x}}_{a}} \kappa_{(h)}^{*}: \coprod_{(h) \in \mathscr{T} \underline{\underline{x}}_{a}} H^{*}\left(\mathrm{X}\left(\kappa_{\mathscr{T}}(h)\right)\right) \rightarrow \coprod_{(h) \in \mathscr{T} \underline{\underline{x}}_{a}} H^{*}\left(\underline{\mathrm{X}}_{\mathfrak{a}}(h)\right) .
$$

As the manifold case, $\mid \kappa^{*}$ is injective. Denote the image of $\mid \kappa^{*}$ by $\mathscr{K}$.

As we have done in $\$ 5.4 .2$, we fix a basis $\sigma_{\star}$ and its dual $\sigma^{\star}$ for $H_{C R}^{*}(\mathrm{~S})$. Then we get a basis $\Sigma_{\star}$ and its dual $\Sigma^{\star}$ for $H_{C R}^{*}(\mathrm{Z})$. Let $\mathscr{R}_{\Sigma_{\star}}^{*}\left(\underline{\mathrm{X}}_{a} \mid \mathrm{Z}\right)$ be the set of admissible relative $\Sigma_{\star}$-data of $\left(\underline{\mathrm{X}}_{\mathfrak{a}} \mid \mathrm{Z}\right)$. We denote by $\mathscr{R}_{\Sigma_{\star}, \mathscr{K}}^{\bullet}\left(\underline{\mathrm{X}}_{\mathfrak{a}} \mid \mathrm{Z}\right)$ the subset which consists of admissible $\Sigma_{\star}$-data $\mathbf{R}^{\bullet}(I \mid J)$ such that $I \subseteq \mathscr{K}$. We call such a relative data a $\left(\Sigma_{\star}, \mathscr{K}\right)$-data.

In this section, we give a one-to-one correspondence between $\mathscr{R}_{\Sigma_{\star}, \mathscr{K}}\left(\underline{\mathrm{X}}_{\mathfrak{a}} \mid \mathrm{Z}\right)$ and $\mathscr{A}_{\sigma_{\star}}^{\bullet}(\mathrm{X}, \mathrm{S})$. For the relative pair $\left(\underline{X}_{\mathfrak{a}} \mid Z\right)$ we have the partial ordered set $\left(\mathscr{R}_{\Sigma_{x}}^{\cdot}\left(\underline{X}_{\mathfrak{a}} \mid Z\right), \prec\right)$ by the construction in previous subsection. The partial order " $\prec$ " also gives a partial order on its subset $\mathscr{R}_{\Sigma_{*}, \mathscr{H}}^{\bullet}\left(\underline{\mathrm{X}}_{\mathfrak{a}} \mathrm{Z}\right)$. We transfer this partial order to $\mathscr{A}_{\sigma_{*}}^{\bullet}(\mathrm{X}, \mathrm{S})$ via the correspondence we will construct.

As we have done in previous subsection, we need to consider relative $\left(\Sigma^{\star}, \Sigma_{\star}\right)$-data of $\left(Z_{\infty}|\bar{N}| Z\right)$ with $\bar{N}$ being the trivial weight projectification of the normal bundle of $Z$ in $\underline{X}_{a}$. In the following we will write $\bar{N}$ as $\bar{N}_{Z}$ for emphasis. We also need to consider relative data of $\left(\bar{N}_{\mathfrak{a}} \mid Z\right)$. We denote relative data of it by $\mathbf{R}_{\bar{N}_{a}}$.

6.2.1. Correspondence on data. We give a map from $\mathscr{R}_{\Sigma_{\star}, \mathscr{K}}^{\bullet}\left(\underline{\mathrm{X}}_{\mathfrak{a}} \mid \mathrm{Z}\right)$ to $\mathscr{A}_{\sigma_{\star}}^{\bullet}(\mathrm{X}, \mathrm{S})$. Let $\mathbf{R}^{\bullet}(I \mid J)$ be an admissible relative $\left(\Sigma_{\star}, \mathscr{K}\right)$-data where the relative data is

$$
J=(\mathfrak{r}(J), \mathfrak{i}(J)),
$$

with

$$
\mathfrak{r}(J)=\left(\left(s_{1}, u_{1}\right), \ldots,\left(s_{h}, u_{h}\right)\right), \quad \mathfrak{i}(J)=\left(\beta_{1}, \ldots, \beta_{h}\right)
$$

and $\beta_{k} \in \Sigma_{\star}$. Hence $\breve{\beta}_{k}$ are of the form $\theta_{j_{k}}^{\left(\bar{t}_{k}\right)} \cup H_{\left(\bar{s}_{k}\right)}^{\ell_{k}} \in \Sigma^{\bar{s}_{k}} \subseteq \Sigma^{\star}$ with $\bar{s}_{k}$ the inverse of $s_{k}$ and $\bar{t}_{k}=\pi_{\mathscr{T}}\left(\bar{s}_{k}\right)$.

For the $k$-th relative marking with relative data $\left(s_{k}, u_{k}\right)$ and insertion $\beta_{k}$ we associate it an admissible relative data $\mathbf{R}_{\bar{N}_{a}, k}^{+}\left(I_{S, k} \mid J_{k}^{+}\right)$of $\left(\bar{N}_{a} \mid Z, S\right)$ like the one discussed in $\S 5$ :

(1) The relative marking $\mathrm{q}_{\infty}$ is associated to $\bar{s}_{k}$ with contact order $u_{k}$ hence the homology class $A_{k}=R_{k}[F]$ is fixed with $R_{k}=u_{k}$ (cf. Lemma 5.8); 
(2) The relative insertion is $\check{\beta}_{k}=\theta_{j_{k}}^{\left(\bar{t}_{k}\right)} \cup H_{\left(\bar{s}_{k}\right)}^{\ell_{k}}$;

(3) The absolute marking is mapped to the twisted sector of $\mathrm{S}_{\left(t_{k}\right)}$ with $t_{k}=\pi_{\mathscr{T}}\left(s_{k}\right)$;

(4) The absolute insertion is $\left(\theta_{\left(t_{k}\right)}^{j_{k}} \cup \Theta_{\left(t_{k}\right)}\right) \psi^{c_{k}}$ and $c_{k}$ is determined by $\mathfrak{r}_{k}=\left(s_{k}, u_{k}\right)$ and $\ell_{k}$, i.e. by dimension constraint, where $\Theta_{\left(t_{k}\right)}$ is the Thom form of $S_{\left(t_{k}\right)}$ in $X_{\left(t_{k}\right)}$ and $\theta_{\left(t_{k}\right)}^{j_{k}}$ is the orbifold Poincaré dual of $\theta_{j_{k}}^{\left(\bar{t}_{k}\right)}$ in $H_{C R}^{*}(\mathrm{~S})$.

Therefore

$$
\mathbf{R}_{\overline{\mathrm{N}}_{\mathfrak{a}}, k}^{+}=\left(g=0, A=A_{k}, \mathfrak{t}=\left(t_{k}\right) \mid \mathfrak{r}=\left(\bar{s}_{k}, u_{k}\right)\right)
$$

and

$$
I_{\mathrm{S}, k}=\left(\left(\theta_{\left(t_{k}\right)}^{j_{k}} \cup \Theta_{\left(t_{k}\right)}\right) \psi^{c_{k}}\right), \quad \mathfrak{i}\left(J_{k}^{+}\right)=\left(\theta_{j_{k}}^{\left(\bar{t}_{k}\right)} \cup H_{\left(\bar{s}_{k}\right)}^{\ell_{k}}\right) .
$$

Let $\mathbf{R}_{\overline{\mathrm{N}}_{\mathfrak{a}}}^{+, \bullet}\left(I_{S} \mid J^{+}\right)$be the disjoint union of $\mathbf{R}_{\overline{\mathrm{N}}_{\mathfrak{a}}, k}^{+}\left(I_{\mathrm{S}, k} \mid J_{k}^{+}\right)$. One can directly see that the pair

$$
\left(\mathbf{R}^{\bullet}(I \mid J), \mathbf{R}_{\overline{\mathrm{N}}_{\mathfrak{a}}}^{+, \bullet}\left(I_{\mathrm{S}} \mid J^{+}\right)\right)
$$

is a matched pair. Since $I \in \mathscr{K}$, we have

$$
\mathbf{A}^{\bullet}\left(I_{\kappa} ; I_{S}\right):=\mathbf{R}^{\bullet}(I \mid J) * \mathbf{R}_{\overline{\mathrm{N}}_{\mathfrak{a}}}^{+\bullet}\left(I_{S} \mid J^{+}\right)
$$

with $I_{\kappa}=\left(\mid \kappa^{*}\right)^{-1}(I) \in H_{C R}^{*}(\mathrm{X})$. Moreover $\mathbf{A}^{\bullet}\left(I_{\kappa} ; I_{S}\right) \in \mathscr{A}_{\sigma_{\star}}(\mathrm{X}, \mathrm{S})$. We define

$$
\Psi\left(\mathbf{R}^{\bullet}(I \mid J)\right):=\mathbf{A}^{\bullet}\left(I_{\kappa} ; I_{S}\right) \text {. }
$$

When the relative data $\mathbf{R}^{\bullet}(I \mid J)$ has empty relative insertions, i.e.

$$
\mathfrak{r}=\varnothing, \quad J=\varnothing,
$$

we must have $A \cdot[\mathrm{Z}]=0$. We define the corresponding absolute data to be

$$
\Psi\left(\mathbf{R}^{\bullet}(I \mid \varnothing)\right):=\mathrm{A}^{\bullet}\left(I_{\kappa} ; \varnothing\right)=\mathrm{A}^{\bullet}\left(I_{\kappa}\right)
$$

with $\mathbf{A}^{\bullet}=\left(g, \kappa_{*} A, \mathfrak{t}\right)$.

The above construction gives us a map

$$
\Psi: \mathscr{R}_{\Sigma_{\star}, \mathscr{K}}^{\bullet}\left(\underline{X}_{\mathfrak{a}} \mid \mathrm{Z}\right) \rightarrow \mathscr{A}_{\sigma_{\star}}^{\bullet}(\mathrm{X}, \mathrm{S}) .
$$

We next construct a left inverse map of $\Psi$.

Lemma 6.9. Given an absolute insertion relative to $S$ of the form $I_{S}=\left(\theta_{(t)}^{j} \cup \Theta_{(t)}\right) \psi^{c}$, there exists a unique admissible relative data $\mathbf{R}_{\bar{N}_{\mathfrak{a}}}^{+}\left(I_{\mathrm{S}} \mid J^{+}\right)=\mathbf{R}^{+}\left(\left(\theta_{(t)}^{j} \cup \Theta_{(t)}\right) \psi^{c} \mid \theta_{j}^{(\bar{t})} \cup H_{(s)}^{\ell}\right)$ of $\left(\overline{\mathrm{N}}_{\mathfrak{a}} \mid \mathrm{Z}, \mathrm{S}\right)$, whose invariant is nonzero and homology class is a fiber class of $\overline{\mathrm{N}}_{\mathfrak{a}} \rightarrow \mathrm{S}$.

Proof. By Theorem 5.3 and Lemma 5.8, the number $c$ and the twisted sector $t$ uniquely determine the topological data

$$
\mathbf{R}_{\overline{\mathrm{N}}_{\mathfrak{a}}}^{+}=(g=0, A=R[F], \mathfrak{t}=(t) \mid \mathfrak{r}=(s, u)),
$$

with $u=R$. We assign the insertions

$$
I_{\mathrm{S}}=\left(\theta_{(t)}^{j} \cup \Theta_{(t)}\right) \psi^{c} \text {, and } \mathfrak{i}\left(J^{+}\right)=\theta_{j}^{(\bar{t})} \cup H_{(s)}^{\ell},
$$

with $\ell$ being determined by the dimension constraint. So $\mathbf{R}_{\bar{N}_{a}}^{+}\left(I_{S} \mid J^{+}\right)$is an admissible relative data. Its invariant is nonzero follows from Theorem 5.29.

By using this lemma we could get a left inverse of $\Psi$. Actually 
Theorem 6.10. When codim $\mathrm{S} \geq 4, \Psi$ is a bijection. When codim $\mathrm{S}=2$, i.e. $\mathrm{S}$ is a symplectic divisor, $\Psi$ is injective.

Proof. Injectivity. First consider the case with $\mathfrak{r} \neq \varnothing$. Then injectivity follows from the construction $\mathbf{R}_{k}^{+}\left(I_{S, k} \mid J_{k}^{+}\right)$and Lemma 6.9. Secondly, for the case with $\mathfrak{r}=\varnothing$, by the definition (6.8) above, that $\Psi$ is injective is equivalent to that if $\kappa_{*} A_{1}=\kappa_{*} A_{2}$ and $A_{i} \cdot[Z]=0$ then $A_{1}=A_{2}$. The equality $\kappa_{*} A_{1}=\kappa_{*} A_{2}$ implies that $A_{1}-A_{2}$ is a fiber class of $Z \rightarrow S$. Then by $A_{i} \cdot[Z]=0$ we conclude $A_{1}=A_{2}$. This proves the injectivity of $\Psi$.

Surjectivity. We consider the case with codimension greater than 2 . Let $\mathrm{A}^{\bullet}\left(I ; I_{\mathrm{S}}\right) \in \mathscr{A}_{\sigma_{\star}}^{\bullet}(\mathrm{X}, \mathrm{S})$. First consider the case with $I_{\mathrm{S}} \neq \varnothing$. Suppose that

$$
\left.I_{\mathrm{S}}=\left(\left(\theta_{\left(t_{1}\right)}^{j_{1}} \cup \Theta_{\left(t_{1}\right)}\right) \psi^{c_{1}}, \ldots,\left(\theta_{\left(t_{h}\right)}^{j_{h}} \cup \Theta_{\left(t_{h}\right)}\right) \psi^{c_{h}}\right)\right) .
$$

Then for each $I_{S, k}:=\left(\theta_{\left(t_{k}\right)}^{j_{k}} \cup \Theta_{\left(t_{k}\right)}\right) \psi^{c_{k}}$ we may get a unique $\mathbf{R}_{\bar{N}_{\mathfrak{a}}, k}^{+}\left(I_{\mathrm{S}, k} \mid J_{k}^{+}\right)$of $\left(\overline{\mathrm{N}}_{\mathfrak{a}} \mid \mathrm{Z}, \mathrm{S}\right)$ (with homology class $R_{i}[F]$, where $R_{i}$ is determined by $c_{i}$ and $t_{i}$ ) by Lemma 6.9. Let $\mathbf{R}_{\bar{N}_{\mathrm{a}}}^{+\bullet}\left(\tilde{I}_{\mathrm{S}} \mid J^{+}\right)$ being the disjoint union of $\mathbf{R}_{\overline{\mathrm{N}}_{\mathrm{a}}, k}^{+}\left(I_{\mathrm{S}, k} \mid J_{k}^{+}\right)$.

There is an admissible relative data $\mathbf{R}^{\bullet}\left(I^{\kappa} \mid J\right)$ of $\left(\underline{X}_{\mathfrak{a}} \mid Z\right)$ with $I^{\kappa}=\kappa^{*}(I)$ such that

$$
\mathbf{R}^{\bullet}\left(I^{\kappa} \mid J\right) * \mathbf{R}_{\overline{\mathrm{N}}_{\mathfrak{a}}}^{+\bullet}\left(\tilde{I}_{\mathrm{S}} \mid J^{+}\right)=\mathbf{A}^{\bullet}\left(I ; I_{\mathrm{S}}\right)
$$

We next construct it.

Suppose the homology class of $\mathrm{A}^{\bullet}$ is $A$. Let $\kappa^{!} A$ being the class in $\underline{X}_{\mathfrak{a}}$ such that $\kappa_{*}\left(\kappa^{!} A\right)=A$ and $\kappa^{!} A \cdot[Z]=0$. Then the homology class of $\mathbf{R}^{\bullet}$ is

$$
\kappa^{!} A-\sum_{i} R_{i}[F] .
$$

The genus of $\mathbf{R}^{\bullet}$ is the same as the genus of $\mathbf{A}^{\bullet}$. The absolute insertions are $I^{\kappa}:=\kappa^{*}(I)$; the relative insertions are

$$
J=\left(\breve{J}_{1}^{+}, \ldots \breve{J}_{h}^{+}\right) \text {, }
$$

i.e. the dual of $J^{+}$in $\mathbf{R}_{\bar{N}_{\mathfrak{a}}^{+}}^{+\bullet}\left(I_{S} \mid J^{+}\right)$. This gives us the $\mathbf{R}^{\bullet}\left(I^{\kappa} \mid J\right)$. We define $\mathbf{R}^{\bullet}\left(I^{\kappa} \mid J\right)$ to be $\Psi^{-1}\left(\mathrm{~A}^{\bullet}\left(I ; I_{\mathrm{S}}\right)\right)$.

If $I_{\mathrm{S}}=\varnothing$, then we set $\Psi^{-1}\left(\mathbf{A}^{\bullet}(I ; \varnothing)\right)=\mathbf{R}^{\bullet}\left(I^{\kappa} \mid \varnothing\right)$ with $\mathbf{R}^{\bullet}=\left(g, p^{!} A, \mathfrak{t} \mid \mathfrak{r}=\varnothing\right)$.

This $\Psi^{-1}$ is the inverse of $\Psi$.

Remark 6.11. The $\Psi$ is not surjective in codimension 2 case is because of the following fact. For this case $\mathrm{Z} \rightarrow \mathrm{S}$ is not a fiber bundle with generic fiber being a weighted space (or finite quotient of weighted space), but a $\mathbb{Z}_{a_{1}}$-gerbe, where $\mathfrak{a}=\left(\alpha_{1}\right)$ is the blowup weight. Consider an admissible relative data $\mathbf{R}(I \mid J)$ with $\mathbf{R}=(g=0, A, \mathfrak{t} \mid(s, u))$ and $\mathfrak{i}(J)=\beta=\kappa_{(s)}^{*}\left(\theta_{\kappa_{\mathscr{T}}(s)}\right)$. Then we have $A \cdot[\mathrm{Z}]=u$, and

$$
\Psi(\mathbf{R}(I \mid J))=\mathbf{A}\left(I_{\kappa} ; I_{\mathrm{S}}\right)
$$

with $\mathbf{A}=\left(g=0, \kappa_{*} A,\left(\kappa_{\mathscr{T}}(\mathfrak{t}), \kappa_{\mathscr{T}}(s)\right)\right), I_{\kappa}=\left(\mid \kappa^{*}\right)^{-1}(I)$ and $I_{\mathrm{S}}=\left(\theta_{\kappa_{\mathfrak{T}}(s)} \cup \Theta_{\kappa_{\mathfrak{T}}(s)}\right) \psi^{c}$ with $c=$ $\left[u \cdot \alpha_{1}\right]$ by dimension constraint (cf. Proposition 5.11).

Suppose $\operatorname{deg} \theta_{\kappa_{\mathscr{T}}(s)}>0$. Take a $\theta_{\kappa_{\mathscr{T}}(s)}^{\prime}$ such that $\operatorname{deg} \theta_{\kappa_{\mathscr{T}}(s)}^{\prime}=\operatorname{deg} \theta_{\kappa_{\mathfrak{T}}(s)}-1$. Now we construct another admissible absolute data $\mathrm{A}\left(I_{\kappa} ; I_{\mathrm{S}}^{\prime}\right)$ out of the absolute data $\mathrm{A}\left(I_{\kappa} ; I_{\mathrm{S}}\right)$. The topological datum are the same except that the absolute insertion is

$$
I_{\mathrm{S}}^{\prime}=\left(\theta_{\kappa_{\mathscr{T}}(s)}^{\prime} \cup \Theta_{\kappa_{\mathscr{T}}(s)}\right) \psi^{c+1} \neq I_{\mathrm{S}}
$$


Then $\mathbf{A}\left(I_{\kappa} ; I_{\mathrm{S}}^{\prime}\right)$ has no inverse image under $\Psi$. Otherwise suppose $\Psi\left(\mathbf{R}^{\prime}\left(I^{\prime} \mid J^{\prime}\right)\right)=\mathbf{A}\left(I_{\kappa} ; I_{\mathrm{S}}^{\prime}\right)$. Then the homology class $A^{\prime}$ of $\mathbf{R}^{\prime}$ satisfies

$$
\kappa_{*} A^{\prime}=\kappa_{*} A .
$$

Since now $\mathrm{Z} \rightarrow \mathrm{S}$ is just a $\mathbb{Z}_{\alpha_{1}}$-gerbe, we have $A^{\prime}=A$. Then $A^{\prime} \cdot[Z]=A \cdot[Z]=u$. But as above since $\Psi\left(\mathbf{R}^{\prime}\left(I^{\prime} \mid J^{\prime}\right)\right)=\mathbf{A}\left(I_{\kappa} ; I_{\mathrm{S}}^{\prime}\right)$, by the dimension constraint we must have

$$
\left[\alpha_{1} \cdot\left(A^{\prime} \cdot[Z]\right)\right]=\left[\alpha_{1} \cdot u\right]=c+1 .
$$

A contradiction.

6.2.2. Correspondence on invariants. By using $\mathscr{R}_{\Sigma_{x}, \mathscr{K}}^{\bullet}\left(\underline{\mathrm{X}}_{\mathfrak{a}} \mid \mathrm{Z}\right)$ we get a real linear space

$$
\mathbb{R}_{\Sigma_{\star}, \mathscr{K}}=\mathbb{R}\left\{\mathscr{R}_{\Sigma_{\star}, \mathscr{K}}\left(\underline{\mathrm{X}}_{\mathfrak{a}} \mid \mathrm{Z}\right)\right\} .
$$

Denote by

$$
v_{\Sigma_{\star}, \mathscr{K}} \in \mathbb{R}_{\Sigma_{\star}, \mathscr{K}}
$$

the vector with coordinate over $\mathbf{R}^{\bullet}(I \mid J)$ being the invariant $\left\langle\mathbf{R}^{\bullet}(I \mid J)\right\rangle \underline{\mathbf{X}}_{a} \mid \mathbf{Z}$, and the vector

$$
v_{\sigma_{\star}} \in \mathbb{R}_{\Sigma_{\star}, \mathscr{K}}
$$

with coordinate over $\mathbf{R}^{\bullet}(I \mid J)$ being the invariant $\left\langle\Psi\left(\mathbf{R}^{\bullet}(I \mid J)\right)\right\rangle^{\mathrm{X}}$.

Theorem 6.12. There is a linear map $L: \mathbb{R}_{\Sigma_{x}, \mathscr{K}} \rightarrow \mathbb{R}_{\Sigma_{x}, \mathscr{K}}$ having the following properties.

- $L\left(v_{\Sigma_{x}, \mathscr{K}}\right)=v_{\sigma,}$;

- the matrix of $L$ with respect to the basis $\mathscr{R}_{\Sigma_{*}, \mathscr{H}}^{\bullet}\left(\underline{\mathrm{X}}_{\mathfrak{a}} \mathrm{Z}\right)$ is lower triangle and the diagonals are all nonzero.

Hence by Lemma $6.8 \mathrm{~L}$ is invertible.

Proof. Take a relative data $\mathbf{R}^{\bullet}(I \mid J)$, and its correspondence absolute data $\Psi\left(\mathbf{R}^{\bullet}(I \mid J)\right)=\mathbf{A}^{\bullet}\left(I_{K} ; I_{S}\right)$ of $(X, S)$. We degenerate $X$ via blowup along $S$ with weight $\mathfrak{a}$. Then the degeneration formula expresses the absolute invariant

$$
\left\langle\mathbf{A}^{\bullet}\left(I_{\kappa} ; I_{\mathrm{S}}\right)\right\rangle^{\mathrm{X}}=\sum_{\Omega \in \mathscr{T}_{\mathbf{A}^{\bullet}\left(I_{K} ; I_{\mathrm{S}}\right)}\left(I^{+}, I^{-}\right)} c_{\Omega} \cdot\left\langle\mathbf{R}_{-}^{\bullet}\left(I^{-} \mid J_{\mathrm{Z}}^{-}\right)\right\rangle^{\mathrm{X}_{\mathrm{a}}} \mathrm{Z} \cdot\left\langle\mathbf{R}_{\overline{\mathrm{N}}_{\mathrm{a}},+}^{\cdot}\left(I^{+} \mid J_{\mathrm{Z}}^{+}\right)\right\rangle^{\overline{\mathrm{N}}_{\mathrm{a}} \mid \mathrm{Z}} .
$$

We require that $J_{\mathrm{Z}}^{+}$and $J_{\mathrm{Z}}^{-}$belong to $\Sigma^{\star}$ and $\Sigma_{\star}$ respectively, and $I^{-}$belong to $\mathscr{K}$. Hence all $\mathbf{R}_{-}^{\bullet}\left(I^{-} \mid J_{Z}^{-}\right)$in the RHS belongs to $\mathscr{R}_{\Sigma_{*}, \mathscr{K}}\left(\underline{\mathrm{X}}_{\mathfrak{a}} \mid \mathrm{Z}\right)$. Therefore all of them are mapped to absolute datum by $\Psi$.

We define

$$
L\left(\ldots,\left\langle\mathbf{R}^{\bullet}(I \mid J)\right\rangle^{\underline{X}_{a}} \mid \mathrm{Z}, \ldots\right):=\left(\ldots,\left\langle\mathbf{A}^{\bullet}\left(I_{\kappa} ; I_{\mathrm{S}}\right)\right\rangle^{\mathrm{X}}, \ldots\right) .
$$

Then we have $L\left(v_{\Sigma_{\star}, \mathscr{K}}\right)=v_{\sigma_{\star}}$. From (6.11) we see that $c_{\Omega}\left\langle\mathbf{R}_{\overline{\mathrm{N}}_{\mathrm{a}},+}^{\cdot}\left(I^{+} \mid J_{Z}^{+}\right)\right\rangle^{\overline{\mathrm{N}}_{\mathrm{a}} \mid \mathrm{Z}}$ is the coefficient of the matrix of $L$ with respect to the basis $\mathscr{R}_{\Sigma_{x}, \mathscr{K}}^{\bullet}\left(\underline{\mathrm{X}}_{\mathfrak{a}} \mid \mathrm{Z}\right)$ of $\mathbb{R}_{\Sigma_{\mathfrak{x}}, \mathscr{K}}$.

We next prove that the matrix of $L$ is lower triangle and has nonzero diagonal. This consists of 2 steps.

Step 1: Lower triangle. This is equivalent to the statement that all relative datum $\mathbf{R}_{-}^{\bullet}\left(I^{-} \mid J_{Z}^{-}\right)$ on the RHS of (6.11) are lower than $\mathbf{R}^{\bullet}(I \mid J)$.

Consider a pair $\left(\mathbf{R}_{-}^{\bullet}\left(I^{-} \mid J_{Z}^{-}\right), \mathbf{R}_{\overline{\mathrm{N}}_{a},+}^{\bullet}\left(I^{+} \mid J_{Z}^{+}\right)\right)$appearing in the RHS of (6.11). We next show that either $\left(\mathbf{R}_{-}^{\bullet}\left(I^{-} \mid J_{Z}^{-}\right) \prec\left(\mathbf{R}(I \mid J) \text { or }\left\langle\mathbf{R}_{\bar{N}_{a},+}^{\bullet}\left(I^{+} \mid J_{Z}^{+}\right)\right)\right\rangle^{\bar{N}_{a}} \mid \mathbf{Z}=0\right.$. 
$\mathbf{R}_{\bar{N}_{\mathfrak{a}},+}\left(I^{+} \mid J_{Z}^{+}\right)$is a relative data of $\left(\bar{N}_{\mathfrak{a}} \mid Z, S\right)$. We degenerate $\bar{N}_{\mathfrak{a}}$ along $S$ by blowing up $\bar{N}_{\mathfrak{a}}$ along $S$ with weight $\mathfrak{a}$. Then we get

$$
\left(\overline{\mathrm{N}}_{\mathfrak{a}} \mid \mathrm{Z}, \mathrm{S}\right) \stackrel{\text { degenerate }}{\longrightarrow}\left(\mathrm{Z}\left|\overline{\mathrm{N}}_{\mathrm{a}}\right| \mathrm{Z}_{0}\right) \wedge\left(\overline{\mathrm{N}}_{\mathrm{a}}^{+} \mid \mathrm{Z}, \mathrm{S}\right)=\left(\mathrm{Z}_{\infty}\left|\overline{\mathrm{N}}_{\mathrm{Z}}\right| \mathrm{Z}_{0}\right) \wedge\left(\overline{\mathrm{N}}_{\mathfrak{a}} \mid \mathrm{Z}, \mathrm{S}\right)
$$

where

- $\bar{N}_{Z}$ is the trivial weight projectification of the normal bundle of $Z$ in $\bar{N}_{\mathfrak{a}}$,

- $Z_{\infty}$ is the infinite section of $\bar{N}_{Z}$, and corresponds to the original $Z$ in $\bar{N}_{a}$ after we glue back,

- by gluing $Z_{0}$ with the last $Z$ in $\overline{\mathrm{N}}_{\mathfrak{a}}^{+}$we get the original $\left(\overline{\mathrm{N}}_{\mathfrak{a}} \mid \mathrm{Z}, \mathrm{S}\right)$.

Note that $I_{\mathrm{S}}$ is contained in $I^{+}$. Now we apply Lemma 6.9 to $I_{\mathrm{S}} \in I^{+}$and get an admissible relative data

$$
\mathbf{R}_{\overline{\mathrm{N}}_{\mathrm{a}}}^{\cdot}\left(I_{\mathrm{S}} \mid J^{+}\right)=\bigsqcup_{k=1}^{\sharp I_{\mathrm{S}}} \mathbf{R}_{\overline{\mathrm{N}}_{\mathrm{a}}, k}^{+}\left(I_{\mathrm{S}, k} \mid J_{k}^{+}\right)
$$

of $\left(\overline{\mathrm{N}}_{\mathfrak{a}}^{+} \mid Z, S\right)=\left(\overline{\mathrm{N}}_{\mathfrak{a}} \mid Z, S\right)$. By the construction of $\Psi$ we see that the relative data

$$
J^{+}=\left(J_{1}^{+}, \ldots, J_{\sharp I_{S}}^{+}\right)=\breve{J},
$$

is the dual of $J$ in the original $\mathbf{R}^{\bullet}(I \mid J)$.

There is an admissible relative data

$$
\mathbf{R}^{\bullet}\left(J_{Z}^{+}\left|I^{+} \backslash I_{S}\right| J\right)
$$

of $\left(Z_{\infty}\left|\bar{N}_{Z}\right| Z_{0}\right)$ such that

$$
\mathbf{R}^{\bullet}\left(J_{Z}^{+}\left|I^{+} \backslash I_{\mathrm{S}}\right| J\right) * \mathbf{R}_{\overline{\mathrm{N}}_{\mathrm{a}}}^{\bullet}\left(I_{\mathrm{S}} \mid \breve{J}\right)=\mathbf{R}_{\overline{\mathrm{N}}_{\mathrm{a}},+}^{\bullet}\left(I^{+} \mid J_{\mathrm{Z}}^{+}\right) .
$$

In fact, when $\operatorname{codim}_{\mathbb{C}} S \geq 2$, following the same proof in Theorem 6.10 we get this relative data; when $\operatorname{codim}_{\mathbb{C}} S=1$, because that the homology class in $\mathbf{A}^{\bullet}$ is obtained from $\mathbf{R}^{\bullet}$ by $\Psi$, we can also get this relative data.

Hence

$$
\mathbf{R}_{-}^{\bullet}\left(I^{-} \mid J_{Z}^{-}\right) * \mathbf{R}^{\bullet}\left(J_{Z}^{+}\left|I^{+} \backslash I_{\mathrm{S}}\right| J\right) * \mathbf{R}_{\overline{\mathrm{N}}_{\mathrm{a}}}^{\bullet}\left(I_{\mathrm{S}} \mid \breve{J}\right)=\mathbf{A}^{\bullet}\left(I_{\kappa} ; I_{\mathrm{S}}\right)
$$

which implies

$$
\mathbf{R}_{-}^{\bullet}\left(I^{-} \mid J_{Z}^{-}\right) * \mathbf{R}^{\bullet}\left(J_{Z}^{+}\left|I^{+} \backslash I_{\mathrm{S}}\right| J\right)=\mathbf{R}^{\bullet}(I \mid J)
$$

by the construction and injectivity of $\Psi$. Hence when $\mathbf{R}^{\bullet}\left(J_{Z}^{+}\left|I^{+} \backslash I_{S}\right| J\right)$ is not pre- $\overline{\mathrm{N}}$-minimal ${ }^{1}$, $\mathbf{R}_{-}^{\bullet}\left(I^{-} \mid J_{Z}^{-}\right) \prec \mathbf{R}(I \mid J)$.

Now suppose that $\mathbf{R}^{\bullet}\left(J_{Z}^{+}\left|I^{+} \backslash I_{S}\right| J\right)$ is pre- $\bar{N}$-minimal but not $\overline{\mathrm{N}}$-minimal. Then

$$
I^{+}=I_{S}, \quad \mathfrak{r}\left(J_{Z}^{+}\right)=\mathfrak{r}(\breve{J}), \quad \mathfrak{i}\left(J_{Z}^{+}\right) \neq \mathfrak{i}(\breve{J}),
$$

and

$$
\mathbf{R}^{\bullet}\left(J_{\mathrm{Z}}^{+}\left|I^{+} \backslash I_{\mathrm{S}}\right| J\right)=\mathbf{R}^{\bullet}\left(J_{\mathrm{Z}}^{+}|\varnothing| J\right)=\bigsqcup_{k=1}^{\sharp I_{\mathrm{S}}} \mathbf{R}_{k}\left(J_{\mathrm{Z}, k}^{+}|\varnothing| J_{k}\right)
$$

\footnotetext{
${ }^{1}$ Here $\bar{N}$ is the projectification of the normal bundle of $Z$ in $\underline{X}_{\mathfrak{a}}$, hence the $\bar{N}_{Z}$ in (6.12).
} 
are disjoint union of pre- $\bar{N}$-minimal datum of $\left(Z_{\infty}\left|\bar{N}_{Z}\right| Z_{0}\right)$. Then at least one of them are not $\overline{\mathrm{N}}$-minimal. Without loss of generality suppose the first one $\mathbf{R}_{1}^{\bullet}\left(J_{\mathrm{Z}, 1}^{+}|\varnothing| J_{1}\right)$ is not $\overline{\mathrm{N}}$-minimal, i.e. $\mathfrak{i}\left(J_{Z, 1}^{+}\right) \neq \mathfrak{i}\left(\breve{J}_{1}\right)$.

On the other hand, now

$$
\mathbf{R}_{\overline{\mathrm{N}}_{\mathfrak{a}},+}^{\bullet}\left(I^{+} \mid J_{\mathrm{Z}}^{+}\right)=\mathbf{R}^{\bullet}\left(J_{\mathrm{Z}}^{+}|\varnothing| J\right) * \mathbf{R}_{\overline{\mathrm{N}}_{\mathrm{a}}}^{\bullet}\left(I_{\mathrm{S}} \mid \check{J}\right)=\bigsqcup_{k=1}^{\sharp I_{\mathrm{S}}}\left(\mathbf{R}_{k}\left(J_{\mathrm{Z}, k}^{+}|\varnothing| J_{k}\right) * \mathbf{R}_{\overline{\mathrm{N}}_{\mathfrak{a}}, k}^{+}\left(I_{\mathrm{S}, k} \mid \check{J}_{k}\right)\right) .
$$

Consider the first component $\mathbf{R}_{1}\left(J_{\mathrm{Z}, 1}^{+}|\varnothing| J_{1}\right) * \mathbf{R}_{\bar{N}_{a}, 1}^{+}\left(I_{\mathrm{S}, 1} \mid \breve{J}_{1}\right)$. The topological data of this component is the following:

- the genus is zero,

- the homology class is a fiber class, and

- there is one relative marking mapped to the infinity section $Z_{\infty}$ of $\bar{N}_{\mathfrak{a}}$ and one absolute marking mapped to the zero section $S$ of $\bar{N}_{\mathfrak{a}}$.

We degenerate $\left(\overline{\mathrm{N}}_{\mathfrak{a}} \mid \mathrm{Z} ; \mathrm{S}\right)$ as in (6.12) and then the degeneration formula gives us

$$
\left\langle\mathbf{R}_{1}^{\bullet}\left(J_{\mathrm{Z}, 1}^{+}|\varnothing| J_{1}\right) * \mathbf{R}_{\bar{N}_{\mathfrak{a}}, 1}^{+}\left(I_{\mathrm{S}, 1} \mid \breve{J}_{1}\right)\right\rangle^{\bar{N}_{\mathfrak{a}} \mid \mathrm{Z}}=\sum_{J^{\prime} \in \Sigma^{\star}} c_{J^{\prime}} \cdot\left\langle\mathbf{R}_{1}^{\bullet}\left(J_{\mathrm{Z}, 1}^{+}|\varnothing| J^{\prime}\right)\right\rangle^{Z_{\infty}\left|\overline{\mathrm{N}}_{\mathrm{Z}}\right| \mathrm{Z}_{0}} \cdot\left\langle\mathbf{R}_{\bar{N}_{\mathfrak{a}}, 1}^{+}\left(I_{\mathrm{S}, 1} \mid \check{J}^{\prime}\right)\right\rangle^{\bar{N}_{\mathfrak{a}} \mid \mathrm{Z}}
$$

Then by Theorem 5.29 we get

$$
\left\langle\mathbf{R}_{1}^{\bullet}\left(J_{Z, 1}^{+}|\varnothing| J_{1}\right) * \mathbf{R}_{\bar{N}_{\mathfrak{a}, 1}}^{+}\left(I_{\mathrm{S}, 1} \mid \breve{J}_{1}\right)\right\rangle^{\bar{N}_{\mathfrak{a}} \mid \mathrm{Z}}=c_{J_{1}} \cdot\left\langle\mathbf{R}_{1}^{\bullet}\left(J_{\mathrm{Z}, 1}^{+}|\varnothing| J_{1}\right)\right\rangle^{Z_{\infty}\left|\overline{\mathrm{N}}_{\mathrm{Z}}\right| \mathrm{Z}_{0}} \cdot\left\langle\mathbf{R}_{\overline{\mathrm{N}}_{\mathfrak{a}, 1}^{+}}\left(I_{\mathrm{S}, 1} \mid \breve{J}_{1}\right)\right\rangle^{\bar{N}_{\mathfrak{a}} \mid \mathrm{Z}} .
$$

Here $c_{J_{1}}$ is a nonzero constant. However since $\mathfrak{i}\left(J_{\mathrm{Z}, 1}^{+}\right) \neq \mathfrak{i}\left(\breve{J}_{1}\right)$, by Proposition 6.2

$$
\left\langle\mathbf{R}_{1}^{\bullet}\left(J_{Z, 1}^{+}|\varnothing| J_{1}\right)\right\rangle_{\infty}^{Z_{\infty}\left|\bar{N}_{Z}\right| Z_{0}}=0
$$

which implies that the invariant

$$
\left\langle\mathbf{R}_{\bar{N}_{\mathfrak{a}}}^{+}\left(I^{+} \mid J_{Z}^{+}\right)\right\rangle^{\bar{N}_{\mathfrak{a}} \mid \mathrm{Z}}=\prod_{k}\left\langle\mathbf{R}_{k}^{\bullet}\left(J_{Z, k}^{+}|\varnothing| J_{k}\right) * \mathbf{R}_{\bar{N}_{\mathfrak{a}}, k}^{+}\left(I_{\mathrm{S}, k} \mid \check{J}_{k}\right)\right\rangle^{\bar{N}_{\mathfrak{a}} \mid \mathrm{Z}}=0 .
$$

Finally, suppose $\mathbf{R}^{\bullet}\left(J_{\mathrm{Z}}^{+}\left|I^{+} \backslash I_{\mathrm{S}}\right| J\right)$ is $\overline{\mathrm{N}}$-minimal. Then

$$
I^{+}=I_{\mathrm{S}}, \quad \mathfrak{r}\left(J_{\mathrm{Z}}^{+}\right)=\mathfrak{r}(\breve{J}), \quad \mathfrak{i}\left(J_{\mathrm{Z}}^{+}\right)=\mathfrak{i}(\breve{J}),
$$

and

$$
\mathbf{R}^{\bullet}\left(J_{Z}^{+}\left|I^{+} \backslash I_{\mathrm{S}}\right| J\right)=\mathbf{R}^{\bullet}\left(J_{Z}^{+}|\varnothing| J\right)=\bigsqcup_{k=1}^{\sharp I_{\mathrm{S}}} \mathbf{R}_{k}\left(J_{\mathrm{Z}, k}^{+}|\varnothing| J_{k}\right)
$$

are disjoint union of $\overline{\mathrm{N}}$-minimal datum of $\left(\mathrm{Z}_{\infty}\left|\overline{\mathrm{N}}_{\mathrm{Z}}\right| \mathrm{Z}_{0}\right)$. Therefore

$$
\mathbf{R}_{\overline{\mathrm{N}}_{\mathfrak{a}},+}^{\bullet}\left(I^{+} \mid J_{Z}^{+}\right)=\mathbf{R}^{\bullet}\left(J_{Z}^{+}|\varnothing| J\right) * \mathbf{R}_{\overline{\mathrm{N}}_{\mathfrak{a}}}^{\bullet}\left(I_{\mathrm{S}} \mid \check{J}\right)=\mathbf{R}_{\overline{\mathrm{N}}_{\mathfrak{a}}}^{\bullet}\left(I_{\mathrm{S}} \mid \check{J}\right)
$$

Consequently, $\mathbf{R}_{-}^{\bullet}\left(I^{-} \mid J_{Z}^{-}\right)=\mathbf{R}^{\bullet}(I \mid J)$.

Therefore every $\mathbf{R}_{-}^{\bullet}\left(I^{-} \mid J_{Z}^{-}\right)$in the RHS of (6.11) is either lower than or equal to $\mathbf{R}^{\bullet}(I \mid J)$ or has zero coefficient. Therefore the matrix of $L$ is lower triangle.

Step 2: Non-vanishing diagonal. Obviously

$$
\mathbf{R}^{\bullet}(I \mid J) * \mathbf{R}_{\overline{\mathrm{N}}_{\mathrm{a}}}^{\bullet}\left(I_{\mathrm{S}} \mid J^{+}\right)=\mathbf{A}^{\bullet}\left(I_{\kappa} ; I_{\mathrm{S}}\right)
$$


for the admissible relative data $\mathbf{R}_{\overline{\mathrm{N}}_{\mathrm{a}}}^{\bullet}\left(I_{\mathrm{S}} \mid J^{+}\right)$in (6.13), hence $J^{+}=J$. The data $\mathbf{R}_{\overline{\mathrm{N}}_{\mathrm{a}}}^{\bullet}\left(I_{\mathrm{S}} \mid J^{+}\right)$is exactly the proper data we discussed in $\S 5$. By Theorem 5.29 we see that the invariant

$$
\left\langle\mathbf{R}_{\overline{\mathrm{N}}_{\mathfrak{a}}}\left(I_{\mathrm{S}} \mid J^{+}\right)\right\rangle^{\overline{\mathrm{N}}_{\mathrm{a}} \mid \mathrm{Z}} \neq 0
$$

Hence the pair $\left(\mathbf{R}^{\bullet}(I \mid J), \mathbf{R}_{\overline{\mathrm{N}}_{\mathrm{a}}}^{\bullet}\left(I_{\mathrm{S}} \mid J^{+}\right)\right)$appears in the RHS of (6.11).

When $J=\varnothing$, then $I_{\mathrm{S}}=\varnothing$. Then in the degeneration formula for $\mathbf{A}^{\bullet}\left(I_{\kappa} ; \varnothing\right)$, the coefficient of $\mathbf{R}^{\bullet}(I \mid \varnothing)$ is 1 .

This shows that the diagonal are all non-zero. The proof is accomplished.

Remark 6.13. Our theorem generalizes the results of $[36,24]$ on the blowup correspondence of ordinary blowup along symplectic submanifold to the case of weighted blowup along symplectic manifold, and moreover, to the orbifold case.

Remark 6.14. Our results hold for the following more general cases:

- the absolute insertions $I$ in $\mathbf{A}^{\bullet}\left(I ; I_{\mathrm{S}}\right)$ contain psi-classes, i.e. descendent insertions,

- $\mathrm{S}$ is a disjoint union $\mathrm{S}=\coprod_{1 \leq i \leq I} \mathrm{~S}_{i}$ of symplectic sub-orbifolds of $\mathrm{X}$ provided that for $\mathrm{S}_{i}, 1 \leq i \leq I$, there exist symplectic neighborhoods $\mathrm{U}_{i}$ which do not intersect with each other. Then after blowing up, the exceptional divisors are disjoint from each other and have pairwise disjoint tubular neighborhoods.

We may restrict the correspondence on a subset of $\mathscr{R}_{\Sigma_{*}, \mathscr{K}}^{\bullet}\left(\underline{X}_{\mathfrak{a}} \mid \mathrm{Z}\right)$. As in $\S 1.2$, let $[p t]_{\text {ker }} \in$ $H^{\operatorname{dim} \mathrm{X}}(\operatorname{ker} \mathrm{X})$ denote the point class of a connected component of kerX. Then $\mid \kappa^{*}[p t]_{\text {ker }}$ is the point class of the corresponding connected component of ker $\underline{X}_{\mathfrak{a}}$.

We denote by $\mathscr{R}_{\Sigma_{\star}, \mathscr{K}, 0,[p t]_{\text {ker }}}\left(\underline{X}_{\mathfrak{a}} \mid Z\right)$ the subset of $\mathscr{R}_{\Sigma_{\star}, \mathscr{K}}^{\bullet}\left(\underline{X}_{\mathfrak{a}} \mid Z\right)$ which consists of genus zero relative datum with $\mid \kappa^{*}[p t]_{\text {ker }}$ being the first absolute insertion. Then we have the corresponding subset $\mathscr{A}_{\sigma_{\star}, 0,[p t]_{\mathrm{ker}}}(\mathrm{X}, \mathrm{S})$ of $\mathscr{A}_{\sigma_{\star}}^{\bullet}(\mathrm{X}, \mathrm{S})$ which consists of genus zero absolute datum with $[p t]_{\mathrm{ker}}$ being the first insertion. Let

$$
\Psi_{0,[p t]_{\mathrm{ker}}}: \mathscr{R}_{\Sigma_{\star}, \mathscr{K}, 0,[p t]_{\mathrm{ker}}}^{\bullet}\left(\underline{\mathrm{X}}_{\mathfrak{a}} \mid \mathrm{Z}\right) \rightarrow \mathscr{A}_{\sigma_{\star}, 0,[p t]_{\mathrm{ker}}}^{\bullet}(\mathrm{X}, \mathrm{S})
$$

be the restriction of $\Psi: \mathscr{R}_{\Sigma_{\star}, \mathscr{K}}^{\bullet}\left(\underline{X}_{\mathfrak{a}} \mid \mathrm{Z}\right) \rightarrow \mathscr{A}_{\sigma_{\star}}^{\bullet}(\mathrm{X}, \mathrm{S})$. It is a bijection when codimS $\geq 4$ and an injection when codim $S=2$. We also have the corresponding linear space $\mathbb{R}_{\Sigma_{\star}, \mathscr{K}, 0,[p t]_{\mathrm{ker}}}$ and vectors

$$
v_{\Sigma_{\star}, \mathscr{K}, 0,[p t]_{\mathrm{ker}}}, v_{\sigma_{\star}, 0,[p t]_{\mathrm{ker}}} \in \mathbb{R}_{\Sigma_{\star}, \mathscr{K}, 0,[p t]_{\mathrm{ker}}}
$$

The linear map $L$ also restricts on $\mathbb{R}_{\Sigma_{\star}, \mathscr{K}, 0,[p t]_{\text {ker }}}$, which we denote by $L_{0,[p t]_{\text {ker }}}$.

Theorem 6.15. The linear transformation $L: \mathbb{R}_{\Sigma_{\star}, \mathscr{K}} \rightarrow \mathbb{R}_{\Sigma_{\star}, \mathscr{K}}$ restricts to a linear transformation $L_{0,[p t]_{\mathrm{ker}}}: \mathbb{R}_{\Sigma_{*}, \mathscr{K}, 0,[p t]_{\mathrm{ker}}} \rightarrow \mathbb{R}_{\Sigma_{\star}, \mathscr{K}, 0,[p t]_{\mathrm{ker}}}$ and

$$
L_{0,[p t]_{\mathrm{ker}}}\left(v_{\Sigma_{\star}, \mathscr{K}, 0,[p t]_{\mathrm{ker}}}\right)=v_{\sigma_{\star}, 0,[p t]_{\mathrm{ker}}} .
$$

Moreover, the matrix of $L_{0,[p t]_{\mathrm{ker}}}$ with respect to the basis $\mathscr{R}_{\Sigma_{\star}, \mathscr{K}, 0,[p t]_{\mathrm{ker}}}\left(\underline{\mathrm{X}}_{\mathfrak{a}} \mid \mathrm{Z}\right)$ is a lower triangle matrix with non-vanishing diagonals.

In this manner, when $[p t]_{\text {ker }}$ runs through the generators of all components of ker $X$ we get $\# \mathscr{T}_{\text {ker }}^{\mathrm{X}}$ linear transformations.

Remark 6.16. There are also other restrictions of $L$ by taking different subset of $\mathscr{R}_{\Sigma_{\star}, \mathscr{K}}\left(\underline{X}_{\mathfrak{a}} \mid \mathrm{Z}\right)$. For instance, we could replace $[p t]_{\text {ker }}$ by the point class of a general twisted sector of $\mathrm{X}$ not only ker X. 
Let $\mathrm{X}(t)$ be a twisted sector of $\mathrm{X}$. After blowing up $\mathrm{X}$ along $\mathrm{S}$ with weight $\mathfrak{a}$, we get its direct transformation $\underline{X}_{a}(t)$ in $\underline{X}_{a}$ (cf. \$3.3). So $\mid \kappa: \underline{X}_{a}(t) \rightarrow X(t)$. Then the pull back $\mid \kappa^{*}\left([p t]_{(t)}\right)$ of the point class $[p t]_{(t)}$ of $\bar{X}(t)$ is the point class of $\underline{X}_{a}(t)$.

We say that $\mathrm{X}(t)$ is away from $\mathrm{S}$ if $\mathrm{X}(t) \nsubseteq \mathrm{IS}$. Obviously, when $\mathrm{X}(t)$ is away from $\mathrm{S}, \underline{\mathrm{X}}_{a}(t)$ is away from the exceptional divisor $\mathrm{Z}$.

Now we replace the point class $[p t]_{\mathrm{ker}}$ in the construction of $L_{0,[p t]_{\mathrm{ker}}}$ by the point class $[p t]_{(t)}$ of a twisted sector $\mathrm{X}(t)$ that is away from $\mathrm{S}$. Then we also get a linear transformation $L_{0,[p t]_{(t)}}$ : $\mathbb{R}_{\Sigma_{*}, \mathscr{K}, 0,[p t]_{(t)}} \rightarrow \mathbb{R}_{\Sigma_{*}, \mathscr{K}, 0,[p t]_{(t)}}$ and two vectors $v_{\Sigma_{*}, \mathscr{K}, 0,[p t]_{(t)}}, v_{\sigma_{\star}, 0,[p t]_{(t)}} \in \mathbb{R}_{\Sigma_{*}, \mathscr{K}, 0,[p t]_{(t)}}$.

As Theorem 6.15, $L_{0,[p t]_{(t)}}$ satisfies

$$
L_{0,[p t]_{(t)}}\left(v_{\Sigma_{\star}, \mathscr{K}, 0,[p t]_{(t)}}\right)=v_{\sigma_{\star}, 0,[p t]_{(t)}},
$$

and the matrix of $L_{0,[p t]_{(t)}}$ with respect to the basis $\mathscr{R}_{\Sigma_{*}, \mathscr{K}, 0,[p t]_{(t)}}\left(\underline{\mathrm{X}}_{\mathfrak{a}} \mid \mathrm{Z}\right)$ is a lower triangle matrix with non-vanishing diagonals.

R. Wang and the first two authors will study other kinds of restrictions of $L$ in [3].

\section{SYMPLECTIC UNIRULEDNESS}

7.1. Symplectic uniruledness of orbifold groupoids. Let $(X, \omega)$ be a compact symplectic orbifold groupoid. As in $\S 1.2$, for $\operatorname{ker} X=\mid X^{\text {top }}$, we denote by $[p t]_{\text {ker }} \in H^{\operatorname{dim} X}(\operatorname{ker} X)$ the point class of a connected component of $\operatorname{ker} \mathrm{X}$.

Definition 7.1. We say that $(X, \omega)$ is symplectic uniruled if there is a nonzero genus zero Gromov-Witten invariant of the form

$$
\left\langle[p t]_{\mathrm{ker}}, \alpha_{2}, \ldots, \alpha_{k}\right\rangle_{0, A}^{\mathrm{X}} \neq 0
$$

with $k \geq 1$ and $0 \neq A \in H_{2}(|X| ; \mathbb{Z})$.

Remark 7.2. When $\mathrm{X}$ is ineffective, ker $\mathrm{X}$ has several connected components. Then the above definition means that (7.1) holds for the point class of one of the components of kerX.

We also have a characterization of symplectic uniruledness by using descendent invariants as the manifold case (cf. [24, Theorem 4.10]).

Theorem 7.3. (X, $\omega)$ is symplectic uniruled if and only if there is a nonzero, possibly disconnected genus zero descendent orbifold Gromov-Witten invariant of the form

$$
\left\langle[p t]_{\text {ker }}, \tau_{d_{2}} \alpha_{2}, \ldots, \tau_{d_{k}} \alpha_{k}\right\rangle_{0, A}^{\mathrm{X}} \neq 0
$$

with $k \geq 1$ such that the component with the $[p t]_{\mathrm{ker}}$ insertion has nonzero homology class. Here when $\mathrm{X}$ is ineffective, Remark 7.2 also applies.

Proof. In the manifold case, the proof of [24, Theorem 4.10] used only axioms of GromovWitten invariants, the boundary relations of $\psi$-classes, and some properties on genus zero invariants with zero curve class. These axioms and boundary relations of $\psi$-classes are also true for orbifold Gromov-Witten invariants. The orbifold Gromov-Witten theory also has similar properties for genus zero invariants with zero curve class. So the proof of [24, Theorem 4.10] also proves this theorem. 
7.2. Invariance of symplectic uniruledness under weighted blowups. The general correspondence results in $\$ 6.2$ implies that symplectic uniruledness is invariant under weighted blowups. Let $S$ be a compact symplectic sub-orbifold groupoid and $\underline{X}_{\mathfrak{a}}$ be the weighted-a blowup of $\mathrm{X}$ along $\mathrm{S}$.

Theorem 7.4. $X$ is symplectic uniruled if and only if $\underline{X}_{\mathfrak{a}}$ is symplectic uniruled.

Proof. Suppose $X$ is symplectic uniruled, then there is a non-zero class $A \in H_{2}(|X| ; \mathbb{Z})$ and a connected nonzero genus 0 orbifold Gromov-Witten invariant of the form

$$
\left\langle[p t]_{\mathrm{ker}}, \alpha_{2}, \ldots, \alpha_{k}\right\rangle_{0, A^{*}}^{\mathrm{X}}
$$

with $A \neq 0$. By blowing up $X$ along $S$ with weight $\mathfrak{a}$ we degenerate $X$ into $X^{+} \wedge_{z} X^{-}$with $\mathrm{X}^{-}=\underline{\mathrm{X}}_{\mathfrak{a}}$. Then by degeneration formula, we express the invariant $\left\langle[p t]_{\text {ker }}, \alpha_{2}, \ldots, \alpha_{k}\right\rangle_{0, A}^{\mathrm{X}}$ as a combination of possibly disconnected relative invariants of $\left(X^{-} \mid Z\right)=\left(\underline{X}_{a} \mid Z\right)$ and $\left(X^{+} \mid Z\right)$. For those $\alpha_{i}$ supporting away from $\mathrm{S}$, we can put $\alpha_{i}^{-}=\mid \kappa^{*} \alpha_{i} \in \mathscr{K}$. (Note that there would be no such $\alpha_{i}$.) For $[p t]_{\text {ker }}$ we choose $\left([p t]_{\text {ker }}\right)^{-}$to be $\mid \kappa^{*}[p t]_{\text {ker }}$, the point class of the corresponding connected component of $\operatorname{ker} \underline{X}_{\mathfrak{a}}$. Then there must be a nonzero invariants of the form

$$
\left\langle\mathbf{R}^{\bullet}\left(\left|\kappa^{*}\left([p t]_{\mathrm{ker}}\right), \alpha_{i_{1}}^{-}, \ldots, \alpha_{i_{l}}^{-}\right| J\right)\right\rangle \underline{\mathrm{X}}_{\mathfrak{a}} \mid \mathrm{Z},
$$

and the component containing the $\mid \kappa^{*}[p t]_{\text {ker }}$ insertion has nonzero curve class. This nonzero invariant shows that $v_{\Sigma_{\star}, 0,[p t]_{\mathrm{ker}}} \in \mathbb{R}_{\Sigma_{\star}, \mathscr{K}, 0,[p t]_{\mathrm{ker}}}\left(\underline{\mathrm{X}}_{\mathfrak{a}} \mid \mathrm{Z}\right)$ is nonzero. Now we apply Theorem 6.15 to the pair $\left(\underline{X}_{\mathfrak{a}}, S=Z\right)$, i.e. we blowup $\underline{X}_{\mathfrak{a}}$ along $Z$ with weight $\mathfrak{a}=(1)$ to get $\underline{X}_{\mathfrak{a}} \wedge_{Z} \bar{N}_{Z}$. Then we get a nonzero absolute descendent invariant of $\underline{X}_{a}$ with $\mid \kappa^{*}[p t]_{\text {ker }}$ insertion and nonzero curve class. Since $\mid \kappa^{*}[p t]_{\text {ker }}$ is the point class of a component of $k e r \underline{X}_{\mathfrak{a}}, \underline{X}_{\mathfrak{a}}$ is symplectic uniruled by Theorem 7.3.

Conversely, suppose that

$$
\left\langle[p t]_{\text {ker }}, \beta_{2}, \ldots, \beta_{k}\right\rangle_{0, A}^{\underline{x}_{\mathfrak{a}}} \neq 0
$$

with $A \neq 0$, where $[p t]_{\text {ker }}$ is the point class of a component of $\operatorname{ker} \underline{X}_{a}$. By the linearity of orbifold Gromov-Witten invariants, we can assume that $\beta_{j} \in \mathscr{K}=\mid \kappa^{*}\left(H_{C R}^{*}(\mathrm{X})\right), 1 \leq j \leq l$, and for $l+1 \leq j \leq k, \beta_{j}$ are of the form $\sigma_{j} \cup\left[\mathrm{Z}_{(s)}\right]$, with $\left[\mathrm{Z}_{(s)}\right]$ the Thom class of $\mathrm{Z}_{(s)}$ in $\left(\underline{X}_{\mathfrak{a}}\right)_{(s)}$. Then we apply degeneration formula to the degeneration of $\underline{X}_{\mathfrak{a}}$ given by blowup of $\underline{X}_{\mathfrak{a}}$ along $Z$ with trivial weight $\mathfrak{a}=(1)$, and set $\left([p t]_{\text {ker }}\right)^{+}=0, \beta_{j}^{-}=\beta$ for $1 \leq j \leq l$, and $\beta_{j}^{-}=0$ for $l+1 \leq j \leq k$. There must be a nonzero relative invariant of $\left(\underline{X}_{\mathfrak{a}} \mid Z\right)$ of the form $\left\langle\mathbf{R}^{\bullet}\left([p t]_{\text {ker }}, \varpi \mid J\right)\right\rangle \underline{\mathbf{X}}_{a} \mid \mathrm{Z}$ where $\varpi$ has at most $l$ insertions and all belong to $\mid \kappa^{*}\left(H_{C R}^{*}(\mathbf{X})\right), J$ is a $\Sigma_{\star}$-relative data, and the connected component containing the $[p t]_{\text {ker }}$ insertion has nonzero curve class. This point class $[p t]_{\text {ker }}$ is in fact $\mid \kappa^{*}[p t]_{\text {ker }}$, the pull back of the point class of the corresponding connected component of ker X. So we next apply $L_{0,[p t]_{\text {ker }}}$ in Theorem 6.15 for the pair $(X, S)$, we get a nonzero descendent invariant with $[p t]_{\text {ker }}$ (the point class of the corresponding connected component of ker $\mathrm{X}$ ) insertion and nonzero curve class. So $\mathrm{X}$ is symplectic uniruled by Theorem 7.3.

7.3. ( $t$ )-symplectic uniruledness. We may also consider other kinds of symplectic uniruledness by involving point classes of general twisted sectors of $X$ not only ker $X$. For instance, we could consider the following situation. 
Let $(t) \in \mathscr{T}^{\mathrm{X}}$, and $\mathrm{X}(t)$ be the corresponding twisted sector of $\mathrm{X}$. We say that $\mathrm{X}$ is $(t)$ symplectic uniruled if there is a nonzero (possibly descendent) orbifold Gromov-Witten invariant of the form

$$
\left\langle[p t]_{(t)}, \tau_{d_{2}} \alpha_{2}, \ldots, \tau_{d_{k}} \alpha_{k}\right\rangle_{0, A}^{X} \neq 0
$$

with $0 \neq A \in H_{2}(|X| ; \mathbb{Z})$.

Then by Theorem 6.15 and Remark 6.16, the argument that proves Theorem 7.4 will show the following result.

Theorem 7.5. Let $(t) \in \mathscr{T}^{\mathrm{X}}$. Suppose that $\mathrm{X}(t)$ is away from $\mathrm{S}$. So its direct transformation $\underline{X}_{\mathfrak{a}}(t)$ is away from $Z$. Then $X$ is $(t)$-symplectic uniruled if and only if $\underline{X}_{\mathfrak{a}}$ is $(t)$-symplectic uniruled.

Here by $X(t)$ being away from $S$ means that $X(t) \nsubseteq I S$, see Remark 6.16. This assumption ensure that when we use the degeneration formula, we could choose $[p t]_{(t)}^{-}$to be $[p t]_{(t)}$.

\section{REFERENCES}

[1] D. Abramovich, B. Fantechi, Orbifold techniques in degeneration formulas, Ann. Sc. Norm. Super. Pisa Cl. Sci. (5), 16 (2016), No. 2, 519-579.

[2] A. Adem, J. Leida, Y. Ruan, Orbifolds and stringy topology. Cambridge Tracts in Mathematics 171, Cambridge University Press, 2007.

[3] B. Chen, C.-Y. Du, R. Wang, Orbifold Gromov-Witten theory of weighted blowups, preprint.

[4] B. Chen, C.-Y. Du, R. Wang, Groupoid of morphisms of groupoids, arXiv:1712.05094.

[5] B. Chen, S. Hu, A deRham model of Chen-Ruan cohomology ring of abelian orbifolds, Math. Ann., 336 (2006), 51-71.

[6] B. Chen. A.-M. Li, Symplectic virtual localization of Gromov-Witten invariants, arXiv:math/0610370.

[7] B. Chen, A.-M. Li, S. Sun, G. Zhao, Relative orbifold Gromov-Witten theory and degeneration formula, arXiv:1110.6803.

[8] B. Chen, A.-M. Li, B.-L. Wang, Virtual neighborhood technique for pseudo-holomorphic spheres, arXiv:1306.3276.

[9] B. Chen, A.-M. Li, B.-L. Wang, Gluing principle for orbifold stratified spaces, in Geometry and Topology of Manifolds, A. Futaki, R. Miyaoka, Z. Tang, W. Zhang Eds., Springer Proceedings in Mathematics \& Statistics, Vol. 154, pages 15-58, Springer, Japan, 2016.

[10] W. Chen, Y. Ruan, Orbifold Gromov-Witten theory, Contemp. Math., 310 (2002), 25-85.

[11] W. Chen, Y. Ruan, A new cohomology theory for orbifold, Commun. Math. Phys. 248 (2004), 1-31.

[12] B. Chen, G. Tian, Virtual orbifolds and localization, Acta Math. Sin. (Engl. Ser.), 26 (2010), No. 1, 1-24.

[13] C.-Y. Du, Weighted blowup of Gromov-Witten invariants of orbifold Riemannian surfaces along smooth points (in Chinese), Sci. Sin. Math., 47 (2017), No. 3, 409-422.

[14] C.-Y. Du, B. Chen, R. Wang, Symplectic neighborhood theorem for symplectic orbifold groupoids (in Chinese), Acta Math. Sinica (Chin. Ser.), 61 (2018), No. 2, 217-232.

[15] A. Gathmann, Gromov-Wittem invariants of blowups, J. Algebraic Geom., 10 (2001), No. 3, 399-432.

[16] L. Godinho, Blowing up symplectic orbifolds, Ann. Global Anal. Geom., 2001, 20(2): 117-162.

[17] M. Gromov, Pseudo holomorphic curves in symplectic manifolds, Invent. Math., 82 (1985), 307-347.

[18] T. Graber, R. Pandharipande, Localization of virtual class, Invent. Math., 135 (1999), No. 2, 487-518.

[19] V. Guillemin, S. Sternberg, Birational equivalence in symplectic category, Invent. math., 97 (1989), 485-522.

[20] T. Graber, R. Vakil, Relative virtual localization and vanishing of tautological classes on moduli spaces of curves, Duke Math. J., 130 (2005), No. 1, 1-37.

[21] J. Hu, Gromov-Witten invariants of blowups along points and curves, Math. Z., 233 (2000), No. 4, 709-739.

[22] J. Hu, Gromov-Witten invariants of blowups along surfaces, Compos. Math., 125 (2001), 345-352.

[23] W. He, J. Hu, Orbifold Gromov-Witten invariants of weighted blowup at smooth points, Acta Math. Sin. (Engl. Ser.), 31 (2015), No. 5, 825-846.

[24] J. Hu, T.-J. Li, Y. Ruan, Birational cobordism invariance of uniruled symplectic manifolds, Invent. Math., 172 (2008), 231-275.

[25] J. Hu, Y. Ruan, Positive divisors in symplectic geometry, Sci. China Math., 56 (2013), No. 6, 1129-1144. 
[26] E. Ionel, T. Parker, Relative Gromov-Witten invariants, Ann. of Math. (2), 157 (2003), No. 1, 45-96.

[27] E. Ionel, T. Parker, The symplectic sum formula for Gromov-Witten invariants, Ann. of Math. (2), 159 (2004), No. 3, 935-1025.

[28] J. Kollár, Low degree polynomial equations: arithmetic, geometry and topology, In European Congress of Mathematics, Vol. I (Budapest, 1996), volume 168 of Progr. Math., pages 255-288, Birkhäuser, Basel, 1998.

[29] M. Kontsevich, Enumeration of rational curves via torus action, in The Moduli Space of Curves, R. Dijkgraff, C. Faber, and G. van der Geer, eds., pages 335-368, Birkhäuser, Basel, 1995.

[30] J. Li, Stable morphisms to singular schemes and relative stable morphisms, J. Differential Geom., 57 (2001), 509-578.

[31] J. Li, A degeration formula of GW-invariants, J. Differential Geom., 60 (2002), 199-293.

[32] Y.-P. Li, H.-W. Lin, C.-L. Wang, Flops, motives, and invariance of quantum rings, Ann. of Math., 172 (2010), No. 1, 243-290.

[33] A.-M. Li, Y. Ruan, Symplectic surgery and Gromov-Witten invariants of Calabi-Yau 3-folds, Invent. math., 145 (2001), No. 1, 151-218.

[34] T.-J. Li, Y. Ruan, Symplectic birational geometry, in New perspectives and challenges in symplectic field theory, 307-326, CRM Proceedings \& Lectures Notes Volum. 49, M. Abreu, F. Lalonde, and L. Polterovich, eds., Amer. Math. Soc., Providence, RI, 2009.

[35] T.-J. Li, Y. Ruan, Uniruled symplectic divisors, Commun. Math. Stat., 1 (2013), No. 2, 163-212.

[36] D. Maulik, R. Pandharipande, A topological view of Gromov-Witten theory, Topology, 45 (2006), 887-918.

[37] D. Mcduff, Examples of simpy-connected symplectic non-Kähler manifolds, J. Differential Geom., 20 (1984), 267-277.

[38] D. McDuff, D. Salamon, J-holomorphic curves and symplectic topology, Colloquium Publications, vol. 52, Amer. Math. Soc., Providence, RI, 2004.

[39] I. Moerdijk, D. A. Pronk, Orbifolds, sheaves and groupoids, K-Theory, 12 (1997), No. 1, 3-21.

[40] Y. Ruan, Virtual neighborhoods and pseudo-holomorphic curves, in Proceedings of 6th Gökova GeometryTopology Conference, Turkish J. Math., 23, pages 161-231, Sci. Tech. Res. Council Turkey, Ankara, 1999.

[41] I. Satake, On a generalization of the notion of manifold, Proc. Nat. Acad. Sci. U.S.A., 42 (1956), 359-363.

[42] I. Satake, The Gauss-Bonnet theorem for V-manifolds, J. Math. Soc. Japan, 9 (1957), No. 4, 464-492.

[43] Z. Tian, Symplectic geometry of rationally connected threefolds, Duke. Math. J., 161 (2012), 803-843.

[44] Z. Tian, Symplectic geometry and rationally connected 4-folds, J. Reine Angew. Math. (Crelle's Journal), 2015 (2015), 221-244.

[45] C. Voisin, Rationally connected 3-folds and symplectic geometry, in Géométrie Différentielle, Physique Mathématique, Mathématiques et Société, II, Astérisque, 322, pages 1-21, Soc. Math. France, Montrouge, 2008.

Department of Mathematics and Yangtze Center of Mathematics, Sichuan University, 610065, Chengdu, CHINA.

E-mail address: bohui@cs.wisc.edu.

Department of Mathematics, Sichuan Normal University, 610068, Chengdu, China.

E-mail address: cyd9966@hotmail.com

Department of Mathematics, Sun Yat-Sen University, Guangzhou, 510275, China

E-mail address: stsjxhu@mail.sysu.edu.cn 